\title{
Ethische Grundlagen und gesellschaftliche Perspektiven einer artgerechteren landwirtschaftlichen Nutztierhaltung
}

\author{
Dissertation \\ zur Erlangung des Doktorgrades \\ der Fakultät für Agrarwissenschaften \\ der Georg-August-Universität Göttingen
}

vorgelegt von Kira von Knoop

geboren in Münster

Göttingen, Dezember 2014 
D7

1. Referent: Prof. Dr. Rainer Marggraf

2. Korreferentin: PD Dr. Micha Strack

Tag der mündlichen Prüfung: 12.02.2015 
Wir selbst müssen die Veränderung sein, die wir in der Welt sehen wollen.

- Mahatma Gandhi 


\section{Zusammenfassung}

Die landwirtschaftliche Tierhaltung ist in den letzten Jahren vermehrt in den Fokus öffentlicher und politischer Diskussionen gerückt. Die Auslöser waren zum einen zahlreiche Lebensmittelskandale wie nachgewiesenes Dioxin in Eiern oder ungekennzeichnetes Pferdefleisch in Fertigprodukten, zum anderen aber auch ein zweifelhafter moralischer Umgang mit Tieren in der landwirtschaftlichen Nutztierhaltung.

Im ersten Teil der vorliegenden Arbeit werden verschiedene tierethische Theorien erläutert und diskutiert. Dabei wird auf die Diskussion zur Rechtfertigung der Nutzung von Tieren für menschliche Zwecke, insbesondere für die Lebensmittelproduktion, eingegangen. Besonders problematisch erscheint aus ethischer Sicht zum aktuellen Zeitpunkt, dass Nutztiere in modernen Tierhaltungssystemen leiden müssen.

Darauf aufbauend wird im zweiten Teil eine quantitative Studie vorgestellt, die sich mit der Einstellung zur landwirtschaftlichen Nutztierhaltung von Studenten aus verschiedenen Fachdisziplinen beschäftigt. In der 2013 durchgeführten Studie wurden die persönliche Akzeptanz und die eingeschätzte Validität von Präferenzund Motivationstests zur Verbesserung von Haltungsbedingungen abgefragt. Nachdem die Probanden ihre Zahlungsbereitschaft (ZB) für die Lebensmittel Eier, Milch und Hackfleisch angegeben hatten, erfolgte die Erfassung der Mehrzahlungsbereitschaft (MZB) für diese Produkte aus Betrieben, in denen die Haltungsbedingungen mit Hilfe von Präferenz- und Motivationstests verbessert wurden. Die Fachdisziplinen ökologische Agrarwissenschaften, Biologie, Landschaftsökologie und Philosophie sind unzufriedener mit den aktuellen Zuständen in der konventionellen landwirtschaftlichen Nutztierhaltung als die Disziplinen Agrarwissenschaften, BWL/VWL und Jura. Die Akzeptanz und die eingeschätzte Validität von Präferenz- und Motivationstests sind jedoch in allen untersuchten Studienfächern gleichermaßen positiv. Für $1 \mathrm{l}$ Milch konnte eine ZB von $0,99 €$ und eine MZB von 0,35 €, für 6 Eier eine ZB von 1,93 € und eine MZB von $0,42 €$ und für 250 g Hackfleisch eine ZB von 2,70 € und eine MZB von 0,57 € ermittelt werden.

Da hinter den verschiedenen Einstellungen zur landwirtschaftlichen Nutztierhaltung unterschiedliche Wertvorstellungen innerhalb der Fachdisziplinen vermutet wurden, 
wurden die Werte der Probanden durch den von Shalom Schwartz entwickelten PVQ- 21 (Portrait Values Questionnaire) erfasst. Der Zusammenhang zwischen den Wertvorstellungen und den Einstellungen zur landwirtschaftlichen Nutztierhaltung ist Gegenstand des dritten Artikels. Mit Hilfe des PVQ-21 konnten die verschiedenen Fachdisziplinen in drei Gruppen mit ähnlichen Wertvorstellungen zusammengefasst werden. Die erste Gruppe bilden Studierende der ökologischen Agrarwissenschaften, Landschaftsökologie, Biologie und Philosophie. Studierende dieser Studienfächer sprechen Tieren ein höheres Bewusstsein zu und sind Veränderungen in der landwirtschaftlichen Nutztierhaltung gegenüber aufgeschlossener. Die zweite Gruppe setzt sich aus Agrarwissenschaftlern, BWL/VWLern und Juristen zusammen. Diese Fachdisziplinen sind hinsichtlich der aktuellen Nutztierhaltungsbedingungen deutlich toleranter eingestellt. Die dritte Gruppe bildet die Fachdisziplin Psychologie. Sie positioniert sich im Wertekreis genau zwischen den ersten beiden Gruppen. 


\section{Danksagung}

Die Fertigstellung einer Dissertation ist von Höhen und Tiefen begleitet, die ohne die Unterstützung einiger Menschen sicherlich weniger intensiv und lehrreich verlaufen wären.

Zu Beginn möchte ich mich bei meinem Doktorvater Professor Dr. Rainer Marggraf für sein Engagement für mein Forschungsgebiet und seine stetige Betreuung bedanken. Er hat mit vielen Ideen und Überlegungen zur Fertigstellung dieser Arbeit beigetragen.

Des Weiteren möchte ich mich bei meiner Zweitprüferin PD Dr. Micha Strack, meinem Zweitprüfer Dr. Holmar Steinfath sowie den Koordinatoren des Studiengangs für Biodiversität und Gesellschaft Dr. Bettina Ross und Dr. Jan Barkmann für ihre organisatorische Begleitung bedanken.

Meinen Kollegen und Freunden gilt besonderer Dank für die zahlreichen konstruktiven Diskussionen und Anregungen. Dabei möchte ich insbesondere meiner Kollegin Katherina Grafl danken, die stets ein offenes Ohr für meine Gedanken hatte. Für die Aufrechterhaltung meiner Motivation und die Gesellschaft an zahlreichen Wochenenden im Blauen Turm möchte ich Christine Rumpf danken.

Ein weiterer Dank geht an meine Familie. Ich bedanke mich bei meinem Vater für das Korrekturlesen meiner Dissertation, bei meinen Geschwistern für den engagierten Beistand und die vielen Ablenkungen sowie bei meiner Mutter für die moralische Unterstützung. Ein besonderer Dank gilt meinem Freund Max, der nicht nur Verständnis für meine Abwesenheit hatte, sondern mich auch durch zahlreiche Diskussionen begleitet und mir geholfen hat meine Gedanken zu formen. 


\section{Inhaltsverzeichnis}

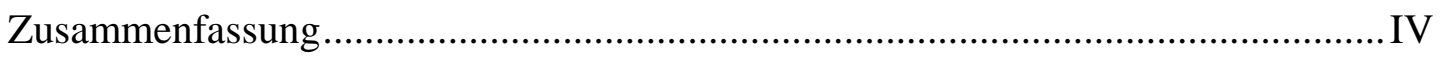

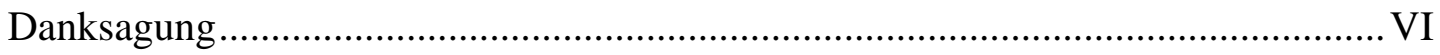

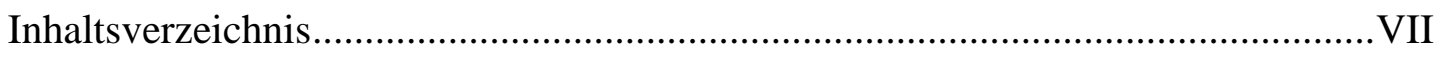

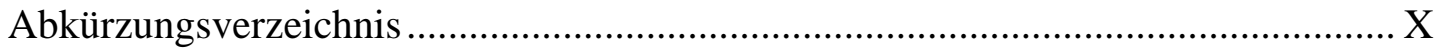

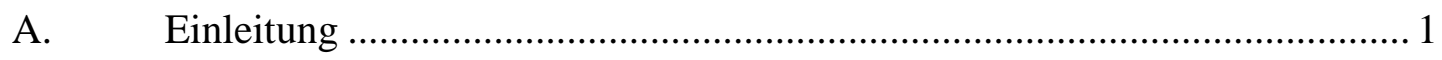

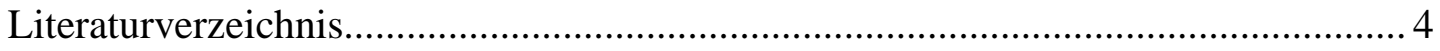

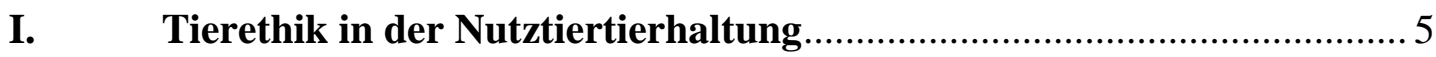

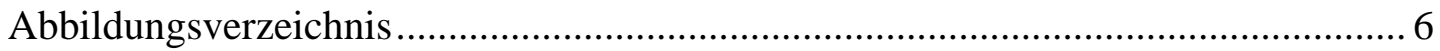

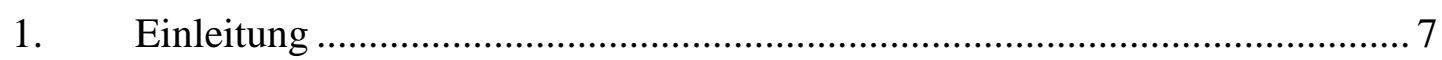

2. Entwicklungen in der Tierschutzpolitik ..................................................... 8

3. Position landwirtschaftlicher Nutztiere..................................................... 11

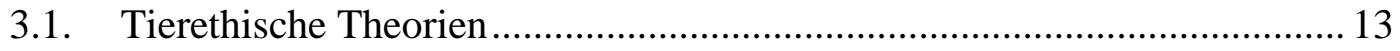

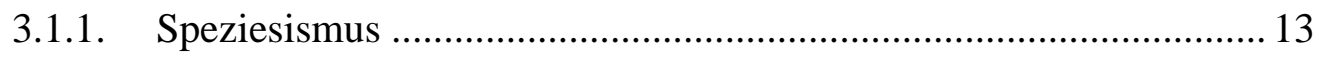

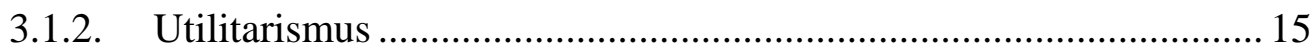

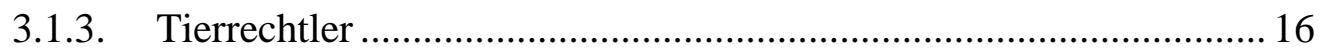

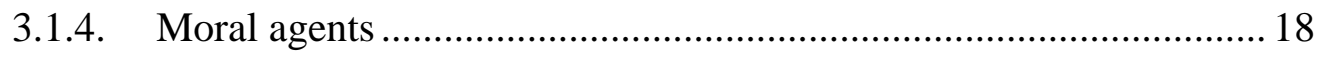

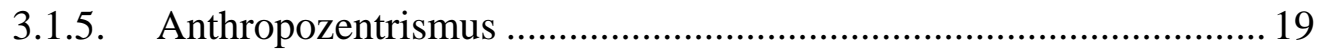

3.1.6. Naturbezogene Vernunftethik .................................................. 20

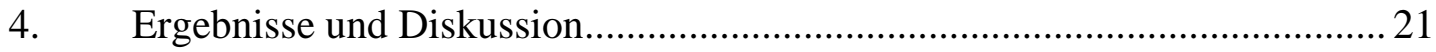

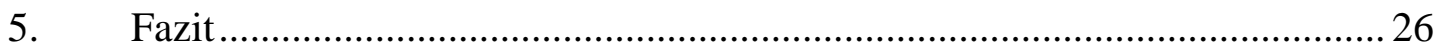

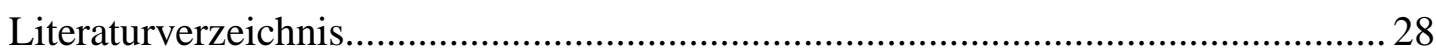

II. Einsatz von Präferenz- und Motivationstests in der Nutztierhaltung.. 33

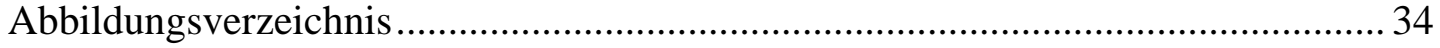

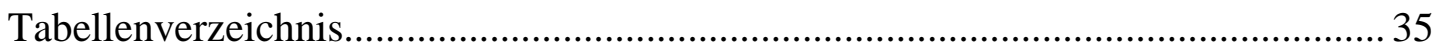

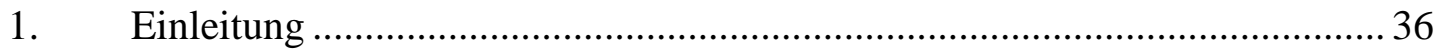

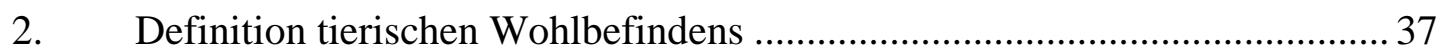

3. Der Präferenz- und Motivationstest ....................................................... 42 
3.1. Hintergrundtheorie zu Präferenz- und Motivationstests ............................ 42

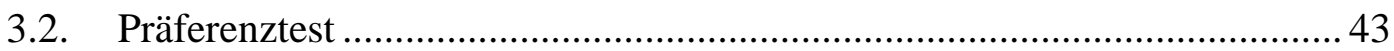

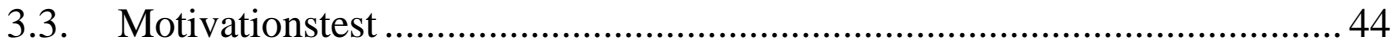

3.4. Motivationstest mit Nerzen ..................................................................... 45

3.5. Kritik an Präferenz- und Motivationstests................................................ 46

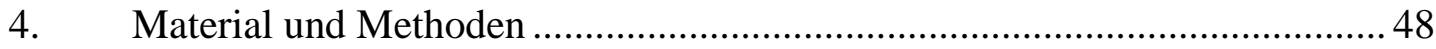

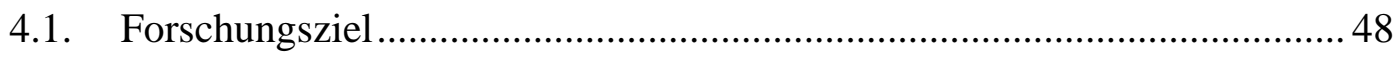

4.2. Fragebogenkonstruktion und Datenerhebung......................................... 49

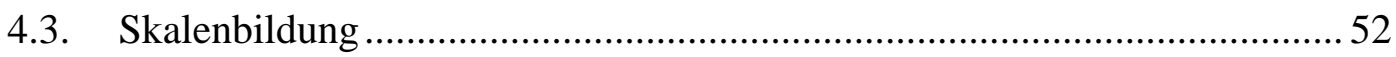

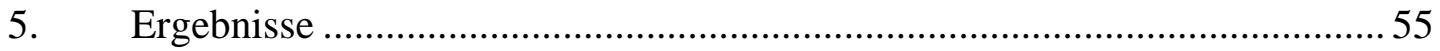

5.1. Beschreibung der Stichprobe..................................................................... 55

5.2. Bewusstseinszuschreibung von Menschen und Tieren .............................. 56

5.3. Einstellung zur aktuellen Tierhaltung und Fleischkonsum ........................ 58

5.4. Einschätzung und Validität von Präferenz- und Motivationstests .............. 62

5.5. Zahlungs- und Mehrzahlungsbereitschaft für tierische Produkte aus mit Präferenz- und Motivationstests optimierter Haltung ......................................... 63

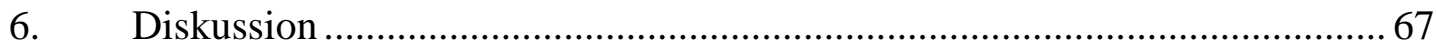

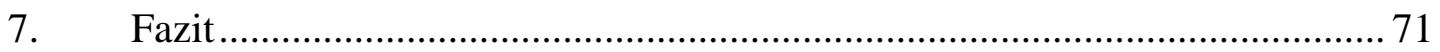

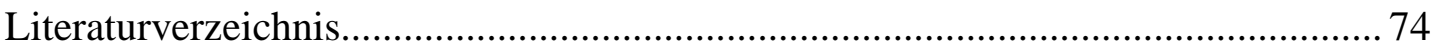

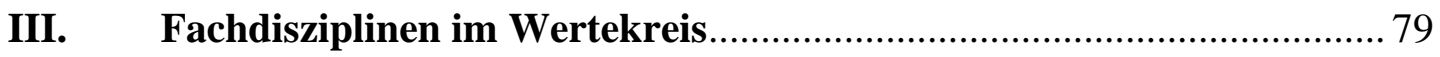

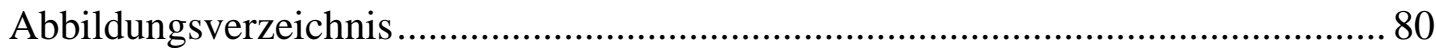

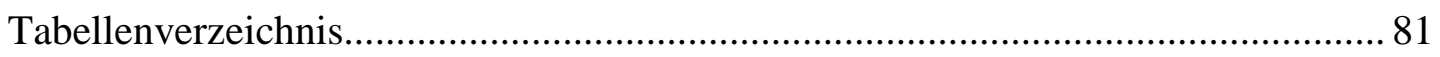

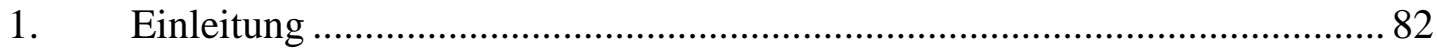

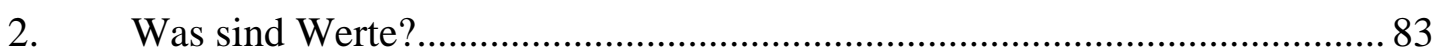

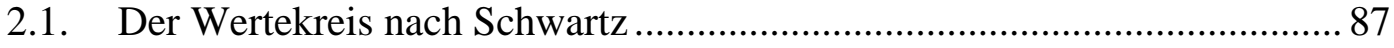

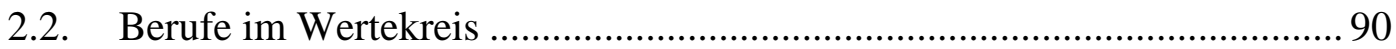

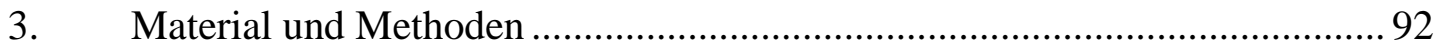

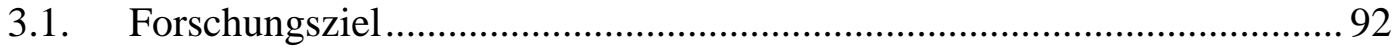

3.2. Fragebogenkonstruktion und Datenerhebung......................................... 92

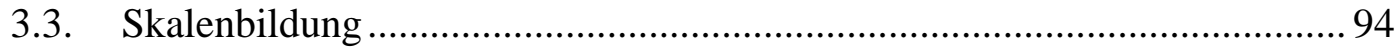

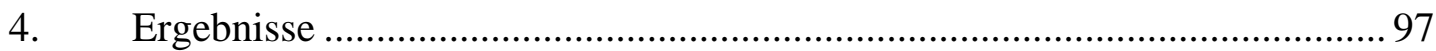




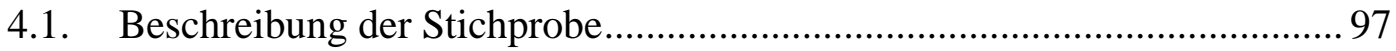

4.2. Einstellungen zur landwirtschaftlichen Nutztierhaltung in verschiedenen

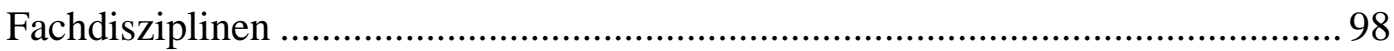

4.3. Studenten unterschiedlicher Fachbereiche im Wertekreis ........................ 101

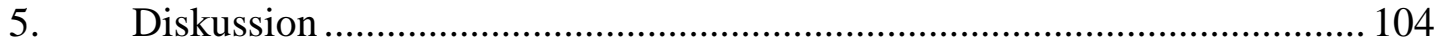

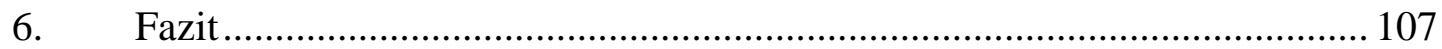

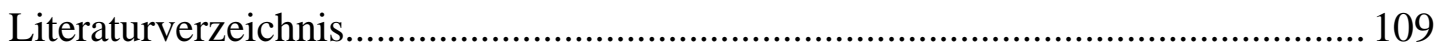

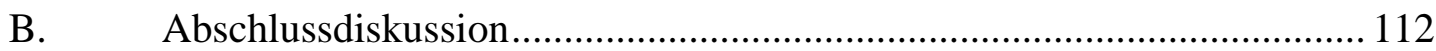

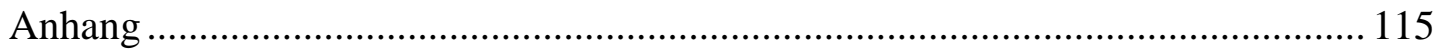




\section{Abkürzungsverzeichnis}

BMELV - Bundesministerium für Ernährung, Landwirtschaft und Verbraucherschutz

BÖLW - Bund ökologische Lebensmittelwirtschaft

BWL - Betriebswirtschaftslehre

dCohen - Effektstärke nach Cohen

IHV - Industrieverband Heimtierbedarf

MT - Motivationstest

MW - Mittelwert

MZB - Mehrzahlungsbereitschaft

$\mathrm{N}$ - Anzahl

p - Signifikanzniveau

PT - Präferenztest

PVQ - Portraits Value Questionnaire

$\mathrm{r}$ - Korrelationskoeffizient

$\mathrm{R}^{2}$ - Bestimmtheitsmaß

SD - Standardabweichung

Sig. - Signifikanz

SVS - Schwartz Value Survey

VWL - Volkswirtschaftslehre

ZB - Zahlungsbereitschaft

$\alpha$ - Cronbachs Alpha

$\beta$ - Regressionskoeffizient 


\section{A. Einleitung}

Das Vertrauen in tierische Produkte ist zunehmend geschwächt. Als Ursache können einerseits Skandale in der Tierproduktion und Fleischverarbeitung und andererseits ein zunehmendes Bewusstsein um die Herstellungsumstände tierischer Produkte identifiziert werden. Das Resultat ist unter anderem eine steigende Anzahl an Vegetariern. 2014 ernährten sich bereits 7,5\% der deutschen Bevölkerung vegetarisch (Statistisches Bundesamt 2014). Hinzu kommt die anhaltende ethische Diskussion um die generelle Nutzung des Tieres zu menschlichen Zwecken. Aus anthropozentrischer Sicht dient das Tier der Bedürfnisbefriedigung des Menschen wohingegen die pathozentrische Umweltethik der Nutzung des Tieres kritischer gegenüber steht. Grundlegend problematisch erscheint aus ethischer Sicht der Umstand, dass Tiere unter den aktuellen Haltungsbedingungen leiden müssen. Demnach sollten die Haltungsbedingungen dahingehend verbessert werden, dass das Leben des Tieres artgerechter verlaufen kann. Dieser Wunsch ist auch bei Konsumenten immer verbreiteter (BMELV 2013).

Im Rahmen der Verbraucherstudie 2014 gaben $48 \%$ der Befragten an, beim Lebensmitteleinkauf darauf $\mathrm{zu}$ achten, dass die eingekauften Produkte aus artgerechter Haltung stammen. 2010 waren es lediglich $36 \%$ (SGS Verbraucherstudie 2014). Aus diesem Wunsch nach Produkten aus artgerechterer Tierhaltung resultieren zum einen steigende Absätze von ökologisch erzeugten Lebensmitteln (BÖLW 2012) und zum anderen erhöhte Anforderungen an landwirtschaftliche Betriebe. Um diesen steigenden Ansprüchen und Bedürfnissen gerecht zu werden, müssen sich die Verfahren in der landwirtschaftlichen Nutztierhaltung weiterentwickeln.

Damit das Wohlbefinden und die Bedürfnisse von Tieren stärker in Haltungsfragen berücksichtigt werden können, muss zunächst eine Möglichkeit gefunden werden, tierische Präferenzen nicht nur stellvertretend durch den Menschen zu erfassen. Mit Hilfe einer direkten Befragung des Tieres zu Haltungsbedingungen könnten diese aktiv in den Entwicklungsprozess zu verbesserten Haltungsstandards integriert werden. Die direkte Befragung des Tieres kann durch Präferenz- oder Motivationstests durchgeführt werden. Unter einem Präferenztest wird die Möglichkeit verstanden, durch gezielte Versuchsanordnungen Handlungspräferenzen von Tieren zu ermitteln. Dem Tier werden beispielsweise mehrere Räume mit 
verschiedenen Einrichtungsgegenständen vorgestellt. Aus der Anzahl bzw. Länge der Besuche bei einem Einrichtungsgegenstand kann abgeleitet werden, wie wichtig oder unwichtig dem Tier dieser Gegenstand ist. Motivationstests sind ähnlich strukturiert wie Präferenztests. Neben der Präferenz soll jedoch zusätzlich erfasst werden, wie hoch die Motivation des Tieres ist, an einen bestimmten Einrichtungsgegenstand zu gelangen. Dazu werden Türen vor die Räume montiert, welche sukzessive mit Gewichten beschwert werden. Anhand der Bereitwilligkeit des Tieres dieses Hindernis zu überwinden und dabei Körperenergie einzusetzen kann die Motivation des Tieres ermittelt werden (Boe et al. 2011, Abou-Ismail, Mahboub 2010).

Der Wunsch nach artgerecht erzeugten Produkten ist jedoch noch nicht bei allen Konsumenten präsent. Einige Verbraucher scheinen mit den aktuellen Herstellungsverfahren zufrieden gestellt zu werden. Die Bedürfnisse der Verbraucher nach kostengünstigen oder qualitativ hochwertigen und artgerecht erzeugten Lebensmitteln könnten auf unterschiedliche Wertvorstellungen der Konsumenten hindeuten.

Daraus haben sich die folgenden Fragestellungen entwickelt:

Welche tierethischen Theorien beschäftigen sich mit der Einstellung zu landwirtschaftlichen Nutztieren und deren Nutzung?

Dazu werden im ersten Teil der Arbeit verschiedene tierethische Theorien vorgestellt. Diese sollen Standpunkte für oder gegen die Nutzung von Tieren in der Landwirtschaft aufzeigen. Im Anschluss werden diese Theorien hinsichtlich ihrer Anwendbarkeit auf die landwirtschaftliche Nutztierhaltung diskutiert.

Wie ist die Einstellung verschiedener Fachdisziplinen zur Verbesserung der landwirtschaftlichen Nutztierhaltung mit Hilfe von Präferenz- und Motivationstests? Besteht eine Mehrzahlungsbereitschaft für Produkte aus Haltungsbedingungen, die mit Präferenz- und Motivationstests verbessert wurden? Kann in den genannten Fragestellungen ein Unterschied zwischen Fachdisziplinen festgestellt werden?

Grundlage des zweiten und dritten Artikels ist eine empirische Studie. Im zweiten Beitrag wird einerseits die Einstellung zu den aktuellen Haltungsbedingungen in der Nutztierhaltung analysiert und andererseits die Akzeptanz der Möglichkeit untersucht, Präferenz- und Motivationstests für die Verbesserung der 
Tierhaltungsbedingungen einzusetzen. Da Verbesserungen in der Nutztierhaltung mit höheren monetären Investitionen im Vergleich zur konventionellen und ökologischen Tierhaltung einhergehen, wird in dieser Studie auch untersucht, ob Verbraucher bereit sind für aus verbesserten Haltungsbedingungen stammende Lebensmittel mehr Geld auszugeben als für momentan erhältliche Produkte. Ein zentraler Schwerpunkt dieses Beitrags ist die Analyse von Einstellungen der Studenten unterschiedlicher Fachdisziplinen zu diesen Themengebieten.

\section{Können die Einstellungen zur landwirtschaftlichen Nutzierhaltung durch unterschiedliche Wertvorstellungen erklärt werden?}

Gegenstand des dritten Beitrags ist die Analyse der Wertvorstellungen der Fachdisziplinen mit Hilfe des von Shalom Schwartz entwickelten Wertekreises. Dazu wurde der verkürzte PVQ-21 verwendet. Der Fokus liegt auf der Darstellung von Gemeinsamkeiten und Unterschieden zwischen mehreren Fachdisziplinen. ${ }^{1}$

\footnotetext{
${ }^{1}$ Es ist geplant, die hier vorliegenden Artikel separat in Fachzeitschriften zu veröffentlichen. Daraus können inhaltliche Überschneidungen entstehen. Zur Verbesserung der Lesbarkeit wird darauf verzichtet, die männliche und weibliche Form zu verwenden. Selbstverständlich sind Frauen und Männer gleichermaßen angesprochen.
} 


\section{Literaturverzeichnis}

Abou-Imail, U., Mahboub, H. 2011: The effects of enriching laboratory cages using varios physical structures on multiple measures of welfare in singly-housed rats, Laboratory Animals, Vol. 45, S. 145-153

Boe, K., Ehrlenbruch, R., Andresen, I. 2011: The preference for water nipples vs. bowls in dairy goats, Acta Veterinaria Scandinavica, Vol. 53, S. 50-55

Bund ökologische Lebensmittelwirtschaft (BÖLW) 2012: Zahlen, Daten, Fakten-Die Bio- Branche 2012, URL: http://www.boelw.de/uploads/pics/ZDF/ZDF_Endversion_120110.pdf, Abrufdatum: 03.12.2014

Bundesministerium für Ernährung, Landwirtschaft und Verbraucherschutz (BMELV) 2013: Ökobarometer 2013 Repräsentative Bevölkerungsbefragung, URL: http://www.bmel.de/SharedDocs/Downloads/Ernaehrung/Oekobarometer_2013 .pdf?_blob=publicationFile, Abrufdatum:03.12.2014

SGS Verbraucherstudie 2014:Vertrauen und Skepsis: Was leitet die Deutschen beim Lebensmitteleinkauf?, SGS Verbraucherstudie

Statistisches Bundesamt 2014: Anzahl der Vegetarier in Deutschland, URL: http://de.statista.com/statistik/daten/studie/173636/umfrage/lebenseinstellung--anzahl-vegetarier/, Abrufdatum 27.11.2014 


\section{Tierethik in der Nutztiertierhaltung}

\section{Inhaltsverzeichnis}

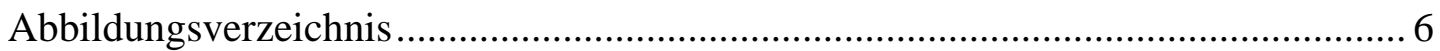

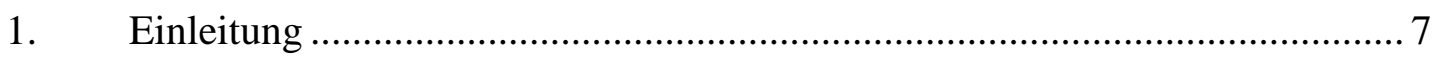

2. Entwicklungen in der Tierschutzpolitik ...................................................... 8

3. Position landwirtschaftlicher Nutztiere ........................................................ 11

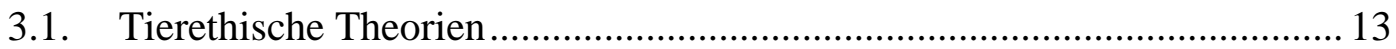

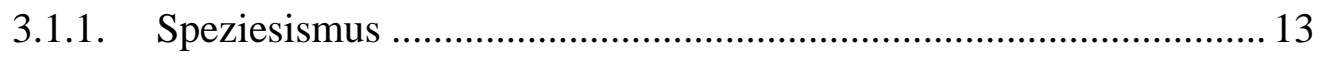

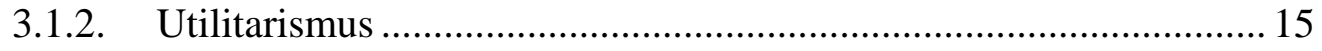

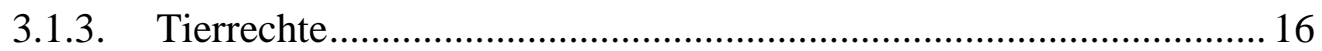

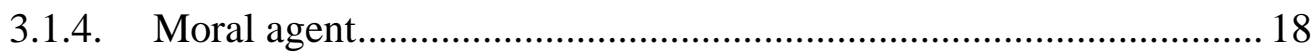

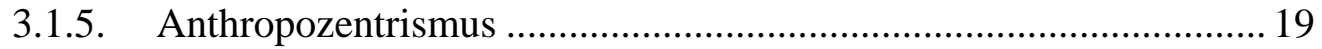

3.1.6. Naturbezogene Vernunftethik .................................................... 20

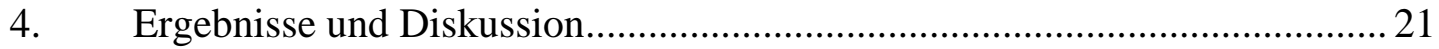

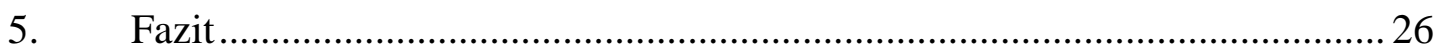

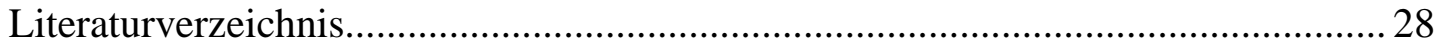




\section{Abbildungsverzeichnis}

Abbildung 1: Einstellungen zu Nutztieren in der agrarwissenschaftlichen Literatur 12

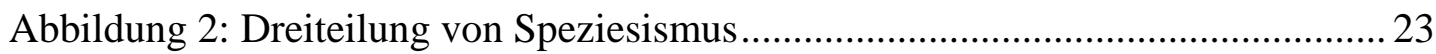




\section{Einleitung}

Das Verhältnis zwischen Menschen und Tieren ist ambivalent. Einige Tierarten werden von uns Menschen in unsere Familien aufgenommen. Sie sind Kinderersatz, Gesprächspartner und manchmal sogar der einzige Anreiz zur Aufrechterhaltung sozialer Kontakte (Coeckelbergh, Gunkel 2014). 2009 wurden in Deutschland ca. 40 Millionen Haustiere gehalten, das entspricht einem Haustier in jedem dritten Haushalt. Im Kontrast dazu stehen die in Deutschland gehaltenen Nutztiere. 2012 wurden 63,1 Millionen Tiere allein zur Fleischproduktion geschlachtet. Das Ergebnis waren 607.261 t Fleisch (Statistisches Bundesamt 2013:5).

Diese Zahlen sprechen bereits dafür, dass Nutztiere in unserer Gesellschaft einen anderen Stellenwert als Haustiere haben. Aber auch unser Sprachgebrauch lässt diesen Schluss zu. In Laboren wird nicht von Mäusen oder Ratten gesprochen, sondern von Tiermaterial und ein Landwirt spricht in der Fachsprache von Großvieheinheiten und nicht von Rindern. In der Terminologie findet eine starke Reduktion des Nutz- und Labortieres auf ihre Verwendung als Ressourcen und Güter statt (Kunzmann, Schmidt 2012:40). Der Gebrauch einer eher technischen Sprache im Zusammenhang mit Tieren führt dazu, diese eher als Objekte anzusehen. Zudem wird dadurch eine Distanz zum Tier geschaffen, die es erlaubt, das Tier als Wesen ohne Emotionen und Bedürfnisse anzusehen (Asquith 2011, Kunzmann, Schmidt 2012:40).

In der Nutztierhaltung werden Themen wie die Enthornung von Kühen, das Kupieren von Ferkelschwänzen und Aspekte zu Haltungsbedingungen häufig auf wissenschaftlicher Ebene diskutiert. Die Veterinärmedizin kann dabei Aufschlüsse über gesundheitliche Aspekte und Hygiene liefern und Verhaltensbiologen klären über arttypisches Verhalten auf. Neben wissenschaftlichen Erläuterungen muss jedoch berücksichtigt werden, dass all diese Themengebiete fundamental ethisch sind. Diese vielfältigen wissenschaftlichen und ethischen Erkenntnisse gilt es zu berücksichtigen, wenn Entscheidungen über tierisches Wohlbefinden getroffen werden (Duncan 2005:483)².

\footnotetext{
${ }^{2}$ Wildtiere und Fische werden in diesen Beiträgen nicht berücksichtigt.
} 


\section{Entwicklungen in der Tierschutzpolitik}

Seitdem sich in Europa Lebensmittelskandale häufen, ist vor allem das Vertrauen in das sensible Produkt Fleisch aber auch in andere tierische Produkte geschwächt. Dazu haben nicht nur Krankheiten wie BSE und die Vogelgrippe beigetragen, sondern in jüngster Zeit auch immer häufiger ein ethisch moralisch zweifelhafter Umgang mit Tieren. Um die Grundlagen des Umgangs mit Tieren zu verstehen ist zunächst ein Exkurs in die Rechtsbereiche nötig, die den Handlungsrahmen für den Umgang mit Tieren darstellen.

Das erste deutsche Tierschutzgesetz wurde am 24.11.1933 mit dem Reichstierschutzgesetz verabschiedet (Kaiser 1934). Es löste die Strafbestimmungen des Reichsstrafgesetzbuchs von 1871 ab, in dem Tierquälerei nur im Sinne eines öffentlichen Ärgernisses angesprochen wurde. Von nun an sollte das Tier und seine Leidensfähigkeit und nicht die Aufrechterhaltung der gesellschaftlichen Moral im Vordergrund stehen. Durch diesen Wechsel wurde der Grundstein des ethischen Tierschutzes gelegt (Lindemann et al. 2010:3).

Am 18. August 1986 ist der ethische Tierschutzgedanke durch die Neuformulierung von $\S 1$ des deutschen Tierschutzrechtes gefestigt worden, indem auch der Schutz des tierischen Wohlbefindens im Tierschutzrecht verankert wurde:

„Zweck dieses Gesetzes ist es, aus der Verantwortung des Menschen für das Tier als Mitgeschöpf dessen Leben und Wohlbefinden zu schützen. Niemand darf einem Tier ohne vernünftigen Grund Schmerzen, Leiden oder Schäden zufügen. “(TSchG 2006)

Durch eine Änderung des Grundgesetzes am 01.08.2002 wurde der Tierschutz schließlich als Staatsziel in das Grundgesetz aufgenommen. Richtungsweisend für diese Änderung war die Begründung des interfraktionellen Gesetzesantrages, in dem der eigenständige Rechtswert und der Verfassungsgehalt des Tierschutzes zum Ausdruck kommen: 
„Die Aufnahme eines Staatszieles Tierschutz trägt dem Gebot eines sittlich verantworteten Umgangs des Menschen mit dem Tier Rechnung. Die Leidensund Empfindungsfähigkeit insbesondere von höher entwickelten Tieren erfordert ein ethisches Mindestmaß für das menschliche Verhalten. Daraus folgt die Verpflichtung, Tiere in ihrer Mitgeschöpflichkeit zu achten und ihnen vermeidbare Leiden zu ersparen. Diese Verpflichtung greift die einfachgesetzlich im Tierschutzgesetz als zentrales Anliegen formulierte Achtung der Tiere auf. Sie umfasst drei Elemente, nämlich: den Schutz der Tiere vor nicht artgemäßer Haltung, vermeidbaren Leiden sowie der Zerstörung ihrer Lebensräume. “ (Deutscher Bundestag 2002).

Auf EU-Ebene wird aktuell ebenfalls an der Verbesserung des Tierschutzes gearbeitet. Im Vertrag über die Arbeitsweise der Europäischen Union vom 30.03.2010 heißt es in Artikel 13:

„Bei der Festlegung und Durchführung der Politik der Union in den Bereichen Landwirtschaft, Fischerei, Verkehr, Binnenmarkt, Forschung, technologische Entwicklung und Raumfahrt tragen die Union und die Mitgliedstaaten den Erfordernissen des Wohlergehens der Tiere als fühlende Wesen in vollem Umfang Rechnung; sie berücksichtigen hierbei die Rechtsund Verwaltungsvorschriften und die Gepflogenheiten der Mitgliedstaaten insbesondere in Bezug auf religiöse Riten, kulturelle Traditionen und das regionale Erbe.“

Durch diesen Artikel ist auf EU-Ebene ein großer Schritt für eine stärkere Berücksichtigung der Tiergerechtheit in der Lebensmittelbranche getan worden. Tiere werden als fühlende Wesen anerkannt, denen dahingehend auch Rechnung getragen werden soll. Vereinzelt wird jedoch der starke Einfluss des römischen Rechtes auf die Verhältnisklärung zwischen Menschen und Tieren kritisiert. In diesem wurde alles, was nicht eindeutig dem Personenstatus zugeordnet werden konnte, als Sache definiert und unterstand den menschlichen Befugnissen. Zunächst 
wurden allerdings nicht nur Tiere in diese Sparte eingegliedert sondern auch Frauen, Kinder und Sklaven (Caspar 1999:42).

Die vorgenommene rechtliche Einstufung des Tieres als Sache hat nach Caspar dem heutigen Tierschutz entgegengewirkt, denn gerade an den Stellen an denen das Tierschutzgesetz das römische Recht rezipiert, nimmt es eine anthropozentrische Stellung ein, welche im eigentlichen nicht das Tier, sondern den Menschen vor den Folgen der Tierquälerei schützt (ibid:43). David Hume verwies bereits 1751 auf die „Gesetze der Menschlichkeit“, in denen er dazu aufforderte Tiere schonend zu nutzen. Kant folgerte daraus, dass die Pflicht zur Tierschonung eine Pflicht des Menschen sich selbst gegenüber sei. Eine Verletzung dieser Pflichten führt zur Abstumpfung und damit zu einer Verringerung des Mitgefühls anderen Menschen gegenüber (Maehle 1999; Kant 1991; Caspar 1998).

Durch die aktuellen Veränderungen im Tierschutzrecht und im Recht zum Umgang mit ihnen wird jedoch deutlich, dass die Leidensfähigkeit und die artgerechte Haltung von Tieren immer stärker berücksichtigt werden.

Aktuell versuchen Politik und Lebensmittelindustrie, das Vertrauen in die landwirtschaftliche Tierproduktion in Deutschland $\mathrm{zu}$ stärken, indem strengere Reglementierungen auferlegt werden und dem Tierschutz ein höherer Stellenwert zugesprochen wird. So wurde 2013 in vier Bundesländern das Verbandsklagerecht für anerkannte Tierschutzorganisationen eingeführt (vgl. Gesetz über das Verbandsklagerecht und Mitwirkungsrechte für Tierschutzvereine vom 25. Juni 2013). Agrarminister suchen einen Ausstieg aus schnabelkürzenden Verfahren bei Legehennen und Nordrhein-Westfalen hat sich dazu entschlossen, das Töten männlicher Eintagsküken von Legerassen nicht mehr im Sinne des §1 des Tierschutzgesetzes als vernünftigen Grund zu dulden. Hessen folgte diesem Beispiel Ende 2014 (Landestierschutzbeauftragte Hessen 2014).

Auf der anderen Seite wird die Anbindehaltung von Nutztieren und Pferden teilweise bis 2020 in Deutschland toleriert, obwohl das Bundesministerium für Landwirtschaft und Ernährung bereits 2009 festgelegt hat, dass eine derartige Einschränkung des natürlichen Bewegungsmusters tierschutzwidrig ist. In der Schweiz ist es dagegen gesetzlich vorgeschrieben, dass Pferde täglich mehrere Stunden freien Auslauf haben müssen. Der Halter muss diese Auslaufzeiten für jedes Pferd nachvollziehbar und prüfbar in einem Auslaufjournal festhalten (BVET 2009). 
Ursula Wolf, eine deutsche Philosophin, die ihren Schwerpunkt unter anderem auf Tierethik legt, gibt zu bedenken, dass zwischen der politischen Einstellung und der Praxis eine große Diskrepanz besteht. Auf der einen Seite werden Tiere als empfindungsfähige Wesen anerkannt und auf der anderen Seiten werden sie massenweise für menschliche Zwecke genutzt und leiden (Wolf 2012:12). Im Bereich der Lebensmittelindustrie ist dies ebenfalls auffällig, da die tatsächlichen Verfahren zur Herstellung von tierischen Produkten im Alltag der Konsumenten nicht widergespiegelt werden (Ach et al. 2013:2).

\section{Position landwirtschaftlicher Nutztiere}

Die Nutzung des Tieres und der Verzehr von tierischen Produkten sind in vielen Gesellschaften weltweit alltäglich. In den modernen Industriegesellschaften, in denen es Lebensmittel im Überfluss gibt, ist Fleisch für jedermann verfügbar. Die Preise für Fleischprodukte werden durch die moderne Tierhaltung immer günstiger, sodass aus dem einstigen Luxusprodukt ein normales Konsumgut geworden ist (Spiller 2008:237).

Die Nutzung von Tieren zur Befriedigung menschlicher Bedürfnisse wird häufig mit dem höheren Grad an Rationalität, Intelligenz, Autonomie oder moralischer Sensitivität des Menschen im Vergleich zum Tier gerechtfertigt (Pluhar 1990:147). Tierisches Leben, so heißt es häufig, ist aufgrund des niedrigeren Entwicklungsniveaus weniger „wertvoll“ als das des Menschen. Tiere sind vernunftlose Wesen und stehen somit in keinem vergleichbaren Verhältnis zum Menschen (Maehle 1999).

Viele Philosophen haben sich mit dem moralischen Konflikt der Nutzung von Tieren für menschliche Zwecke auseinandergesetzt und dennoch ist die landwirtschaftliche Nutztierhaltung seit den Anfängen dieser Diskussion zunächst stetig intensiver geworden (BMELV 2011; BMELV 2012).

Sauerberg und Wierzbitza haben agrarwissenschaftliche Texte ${ }^{3}$ analysiert und sie auf deren Einstellungen und Blickwinkel zu Nutztieren untersucht. Durch diese

\footnotetext{
3 Die Autoren wählten einschlägige Literatur aus, die an Universitäten, Fachhochschulen und
} Berufsschulen verwendet wird. 
Betrachtung konnten sieben mögliche Einstellungen abgeleitet werden, welche typische Auffassungen zu Nutztieren in der Agrarwissenschaft repräsentieren.

\section{Abbildung 1: Einstellungen zu Nutztieren in der agrarwissenschaftlichen Literatur}

Produkt

1. Nutztiere als Produkte: Das Tier wird vorwiegend als Produkt angesehen. Betriebswirtschaftliche Faktoren wie Input-Output Verhältnisse, Energiewerte und technische Optimierung dominieren dieses Tierbild.

2. Körperliche Funktionalität: Die physiologische Funktionalität des Tieres steht im Vordergrund. Orientierung bieten Faktoren wie Gesundheit, Fruchtbarkeit und Ernährungszustand.

3. Bedürfnisbefriedigung: Den körperlichen Bedürfnissen des Tieres soll nachgekommen werden um die Produktqualität zu verbessern. Eine Steigerung des Wohlbefindens für eine bessere Lebensqualität allein steht nicht im Vordergrund.

4. Tierschutz: Der Schutz des Tieres ist genauso wichtig wie die betriebswirtschaftliche Effizienz.

5. Nutztier als Lebewesen: Das Nutztier wird als individuelles Lebewesen mit sozialem Handeln angesehen.

6. Nutztiere sind Teil der Evolution: Menschen und Tiere sind aus einer Co-Evolution entstanden, sie sollten gleichermaßen als Bestandteile der Natur angesehen werden.

7. Nutztier als Subjekt: Tiere werden als beseelte Wesen mit Präferenzen und Abneigungen angesehen. Sie haben Gefühle und können Leiden.

Subjekt 
Die Autoren fanden heraus, dass wissenschaftliche Publikationen in der Agrarwissenschaft zu einem Großteil das Tier als ein Produkt einstufen und sich auf die ökonomische Relevanz des Tieres beschränken. Texte, die das Tier als Subjekt beschreiben sind seltener. Eine Ausnahme bilden hier Veröffentlichungen zur ökologischen Tierhaltung. Bei der Außendarstellung von konventionellen und ökologischen Dienstleistern wird der Fokus jedoch nach wie vor auf das Produkt Tier gelegt. Alle Verbesserungen, die von Betrieben durchgeführt werden zielen insbesondere auf die Optimierung der Produktqualität und die Wirtschaftlichkeit des Herstellungsprozesses. Die aktuelle Position der landwirtschaftlichen Nutztiere umfasst nach dieser Einteilung die ersten drei Gliederungspunkte. Die Kategorien 4. bis 7. werden bislang nur theoretisch angesprochen, eine praktische Umsetzung ist nur vereinzelt in einigen Ausnahmebetrieben zu beobachten (Sauerberg, Wierzbitza 2013:91).

Im folgenden Kapitel sollen verschiedene tierethische Theorien vorgestellt werden, die für oder gegen eine gleiche Interessensberücksichtigung von Menschen und Tieren sprechen. Es werden verschiedene Theorien dargelegt um diese angewendet auf die landwirtschaftliche Nutztierhaltung zu diskutieren.

\subsection{Tierethische Theorien}

\subsubsection{Speziesismus}

Der Begriff Speziesismus wurde 1970 durch den britischen Psychologen Richard Ryder geprägt. Verbreitet wurde dieser jedoch hauptsächlich durch den australischen Philosophen und Ethiker Peter Singer. Nach Singer stellen Speziesisten die Interessen der Mitglieder der eigenen Spezies über die Interessen der Mitglieder einer anderen. Sie begründen die Höherbewertung der menschlichen Interessen allein durch die Zugehörigkeit zur Spezies Homo sapiens (Singer 2006:3).

Die Diskriminierung eines leidenden Tieres ist nach Singer mit der Diskriminierung eines Menschen aufgrund des Geschlechtes oder der Nationalität gleichzusetzen. Der Speziesismus wird aus diesem Grund häufig auf eine Stufe mit Rassismus und Sexismus gestellt, da die Grenze zwischen Berücksichtigung und NichtBerücksichtigung anhand eines Merkmals, der Zugehörigkeit zu einer Spezies, gezogen wird (Singer 2002). 
Descartes Tierautomatentheorie kann beispielsweise als speziesistisch angesehen werden (Horta 2010:257). Descartes vertritt die Meinung, dass alle Wesen, die nicht der Spezies des Homo sapiens angehören gesellschaftlich nicht berücksichtigt werden müssen. Sie haben keine Empfindungen und sind somit zu vernachlässigen. Tiere sind Descartes zufolge seelenlos und funktionieren ähnlich wie ein Uhrwerk. Schmerzreaktionen bei durchgeführten Vivisektionen ${ }^{4}$ erklärte er durch mechanische Reaktionen, die durch Schnitte ausgelöst wurden (Pollack 2007:19). Eine ähnliche Position vertritt auch Immanuel Kant. Der deutsche Philosoph spricht Tieren zwar ein Bewusstsein zu, jedoch keinen Eigenwert ${ }^{5}$. Aus diesem Grund müssen Tiere auch nicht moralisch berücksichtigt werden (Kant 2006). In einer wissenschaftlichen Studie wurde jedoch herausgefunden, dass einige Tiere genau wie wir Menschen einen Geist besitzen. Eine andersartige Behandlung von Tieren aufgrund einer fehlenden Seele ist somit nicht mehr zulässig (Buschka; Rouamba 2013:249).

Speziesismus wird von vielen Tierethikern stark kritisiert, da die Berücksichtigung eines Individuums nicht von Äußerlichkeiten oder Fähigkeiten abhängig zu machen ist. Das Prinzip der Gleichheit bietet den Grundstein für alle Argumente gegen Rassismus und Sexismus. Im Einklang mit dieser Argumentationsgrundlage ist auch der Speziesismus zu hinterfragen (Singer 1997:19).

„Der Tag mag kommen, an dem der Rest der belebten Schöpfung jene Rechte erwerben wird, die ihm nur von der Hand der Tyrannei vorenthalten werden konnten. Die Franzosen haben bereits entdeckt, dass die Schwärze der Haut kein Grund ist, ein menschliches Wesen hilflos der Laune des Peinigers auszuliefern. Vielleicht wird eines Tages erkannt werden, dass die Anzahl der Beine, die Behaarung der Haut oder die Endung des Kreuzbeins ebenso wenig Gründe dafür sind ein empfindliches Wesen diesem Schicksal zu überlassen. “ (Bentham1989; zitiert nach Singer 1997:26).

Der spanische Moralphilosoph und einer der stärksten Kritiker des Speziesismus Oscar Horta gibt zu bedenken, dass der Begriff Speziesismus auf keinen Fall

\footnotetext{
${ }^{4}$ Sektion am lebendigen, unbetäubten Tier.

${ }^{5}$ Der Eigenwert des Tieres kann als Gegensatz zum Nutzwert angesehen werden und ist so zu verstehen, dass das Tier einzig durch seine Existenz als wertvoll erachtet wird (Röcklingsberg 2001).
} 
dahingehend missverstanden werden darf, dass grundlegend eine gleiche Behandlung aller Spezies angestrebt werden sollte (Horta 2010). Die Feststellung, dass es eindeutige Differenzen zwischen Menschen und Tieren gibt, impliziert nicht, dass es auch einen Unterschied in ihrer moralischen Bedeutung gibt. Wichtig ist es zu unterscheiden, dass eine unterschiedliche Behandlung von Tieren und Menschen nicht sofort als Speziesismus angesehen werden kann. Speziesismus tritt erst dann auf, wenn Tiere aufgrund ihrer Zugehörigkeit zu ihrer Spezies anders behandelt werden und fehlende Fähigkeiten, wie Sprache und abstraktes oder moralisches Denken, als Rechtfertigungen dafür angesehen werden (Doğan 2011:479).

Eine unterschiedliche Behandlung von Menschen und Tieren ist auch bei gleicher Interessensberücksichtigung nötig, da Menschen und Tiere nicht dieselben Bedürfnisse oder Ansprüche an ihre Umwelt haben. Die Fähigkeit zu Leid und Freude ist eine Voraussetzung dafür überhaupt Interessen zu haben. Eine Bedingung, die zunächst erfüllt sein muss, ehe auf sinnvolle Weise von Interessen gesprochen werden kann. Wenn ein Wesen leidet, kann es keine moralische Rechtfertigung dafür geben, dieses Leiden nicht zu berücksichtigen, ganz gleich was die Natur dieses Wesens ist. Das Prinzip der Gleichheit erfordert, dass das Leiden eines Tieres ebenso viel zählt wie das Leiden eines Menschen. Wenn ein Wesen dagegen nicht fähig ist zu leiden, Freude oder Glück zu empfinden, dann gibt es auch nichts zu berücksichtigen (Singer 2002:26).

\subsubsection{Utilitarismus}

Primäres Ziel der Utilitaristen ist es, den Gesamtnutzen (utility) einer Gesellschaft zu maximieren. Jeremy Bentham, der Begründer des Utilitarismus, definiert „utility“ als „Freude“ und „disutility“ als „Leid“. Er verdeutlicht, dass im Sinne des Utilitarismus alle Lebewesen mit Empfindungsfähigkeit moralisch relevant und zu berücksichtigen sind, da sie genau wie Menschen leiden können (Sandøe et al. 1997:6; Pluhar 1990:148).

„The question is not, 'Can they reason?' nor 'Can they talk? But ,Can they suffer?، “ (Bentham 1780:283). 
Utilitaristen zielen dabei nicht nur auf die Befriedigung von Interessen einzelner empfindungsfähiger Wesen ab. Wichtiger ist ihnen die Bedürfnisbefriedigung der Gesellschaft als Ganzes (Doğan 2011:274). Henry Sidgwick, ein Vertreter des Utilitarismus, verdeutlicht, dass dabei jegliches Leben gleichbedeutend ist.

„Das gute Leben irgendeines Individuums hat vom Standpunkt [...] des Universums nicht mehr Bedeutung als das gute Leben eines jeden anderen“ (Sidwick 1907:382).

Es ist jedoch im klassischen Utilitarismus nicht verboten Tiere zu halten, zu nutzen und zu töten, wenn die folgenden fünf Aspekte beachtet werden:

1. Das Tier hätte unter anderen Umständen nicht existiert.

2. Das Tier hatte ein erfülltes Leben.

3. Der Tod fügt dem Tier keinen körperlichen Schmerz, Angst oder sonstige vermeidbare Leiden zu.

4. Der Tod des Tieres sollte jenen, die ihm nahe stehen kein Leid zufügen.

5. Das Tier wird nach seinem Tod durch ein anderes ersetzt, für das wiederum die Bedingungen 1. bis 4. zutreffen müssen (Pluhar 1990:150).

Singer propagiert dagegen ein weitgehendes Nutzungsverbot von tierischen Produkten und dies nicht weil er gegen die Tötung von Tieren ist. Singer unterstützt die Nutzung von Tieren zu Nahrungsmittelzwecken nur, wenn das Leben des Tieres ohne zugefügte Leiden und Schmerzen war und auch die Tötung so verläuft, dass das Tier keine Schmerzen empfindet (Sandøe et al. 1997:7).

\subsubsection{Tierrechtler}

Henry Salt gilt als Pionier der Tierrechte im späten 19. Jahrhundert. Schwerpunkt von Salts Arbeit war die Frage welche Rechtfertigung der Mensch hat, Tiere anders als Menschen zu behandeln. Salt vertrat den Standpunkt, dass es inkonsistent wäre, Menschen Rechte zuzusprechen und Tieren nicht. Tiere verfügen genau wie Menschen über Charakter, Vernunft und Individualität. Es sei daher eine logische 
Konsequenz Tieren Rechte zu gewähren (Taylor 2003:61). Aus Sicht der Tierrechtler gilt es nicht nur das menschliche und tierische Wohlbefinden als Ganzes zu berücksichtigen. Es ist vielmehr die Pflicht des Menschen jedes Individuum vor dem Tod und anderen Benachteiligungen zu schützen (Sandøe et al. 1997:10).

Von Tierrechtlern wird ebenfalls eingeräumt, dass eine moralische Berücksichtigung von Tieren nicht darauf basieren sollte, dass sie durch ihre Spezieszugehörigkeit grundsätzlich einige Fähigkeiten haben könnten. Ausschlaggebend ist nur, welche Fähigkeiten das Individuum tatsächlich hat und nicht welche es prinzipiell haben könnte. Problematisch ist an dieser Sichtweise jedoch, dass Kleinkinder, Demente oder Behinderte nicht unter die moralische Berücksichtigung fallen, da diese auch nicht über alle Fähigkeiten eines gesunden Erwachsenen verfügen (Anderson 2014:294).

Zahlreiche Tierrechtler lehnen die Nutzung des Tieres für menschliche Zwecke kategorisch ab. Darunter fällt nicht nur die Nutzung für Tierversuche sondern auch das Töten von Tieren zur Fleischproduktion. Einer der bekanntesten Tierrechtler ist Tom Regan. Dieser lehnt jegliche Instrumentalisierung von Säugetieren ab. Eine Nutzung von Tieren ist, ganz gleich was für Interessen dahinter stehen, aus Regans Sicht nicht vertretbar, da alle empfindungsfähigen Wesen in einem gleichen Maß berücksichtigt werden müssen. Darin eingeschlossen sind auch die wissenschaftliche Forschung, die Agrarindustrie sowie die Jagd (Regan 2004).

Die kategorische Ablehnung der Nutzung von Tieren wird jedoch nicht von allen Tierrechtlern konsequent verfolgt. Die konventionelle und industrielle Tierhaltung wird zwar abgelehnt, eine traditionelle Haltung und Nutzung wird jedoch teilweise akzeptiert. Regan lehnt diese Ausnahmeregelung konsequent ab. Er ist der Meinung, dass das ganze System um die Nutzung von Tieren fehlerhaft ist:

„Once we accept this view of animals- as our resource- the rest is as predictable as regrettable. Why worry about their loneliness, their pain, their death. Since animals exist for us to benefit us in one way or another, what harms them really does not matter or matters only if it starts to bother us, makes us feel a trifle uneasy. [...] a little straw, more space and a few companions won't eliminate- won't even touch- the basic wrong that attaches 
to our viewing and treating these animals as our resource." (Regan 2004:179f).

\subsubsection{Moral agents}

Die Begriffe der „moral agents“ (moralische Akteure) und „moral patients“ (moralische Empfänger) wurden von Tom Regan 1983 in seinem Buch „The case for animal rights“ entwickelt. Die Argumentation der „moral agents“ beruht auf der Annahme, dass Menschen Personen oder anders ausgedrückt moralische Akteure sind. Tiere fallen in die Kategorie der „moral patients“. Obwohl sie in der Lage sind, sich moralisch richtig oder falsch zu verhalten, können sie für dieses Verhalten nicht verantwortlich gemacht werden. Ihnen fehlt das Bewusstsein zur Lenkung von moralischem Verhalten. Durch die Verwendung der Begriffe „moral agents“ und „moral patients“ wird zum einen auf die aus der Naturrechtslehre stammende Behauptung Bezug genommen, dass Rechtsgemeinschaften nur unter Vernunftwesen existieren. Zum anderen auf Kants Ausschluss von Tieren aus der „moral Community“ aufgrund der Asymmetrie von Rechten und Pflichten die entstehen würde, wenn Menschen und Tiere die gleichen Rechten haben würden, da Tiere Menschen gegenüber keinerlei Pflichten erfüllen können (Baranzke 2002:319).

Die Frage nach Rechten und Pflichten stellt sich erst dann, wenn ein moralischer Akteur auf ein anderes empfindungsfähiges Wesen stößt. Treffen zwei „moral patients“ aufeinander, so stellt sich die Frage nach moralischer Berücksichtigung gar nicht erst, denn erst der moralische Akteur kann aus einer Situation einen moralischen Status ableiten (Sezgin 2014:40).

Das Problem, das bei dieser Theorie ebenso wie bei Tierrechtlern auftritt ist, dass Kinder und geistig Behinderte nicht als Personen und somit nicht als „moral agents“ angesehen werden. Sie zählen wie Tiere in die Kategorie der moralischen Empfänger und dürften somit genauso wie Tiere behandelt werden (Regan 2004:152ff; Pluhar 1990:148). 


\subsubsection{Anthropozentrismus}

Im Anthropozentrismus steht der Mensch mit seinen Fähigkeiten und Bedürfnissen im Mittelpunkt (Sztybel 2000:182). Die Natur und Tiere sind im Sinne des Anthropozentrismus Ressourcen, die der Bedürfnisbefriedigung des Menschen dienen und keinen Eigenwert haben (Mahlke 2014).

Taylor ist der Meinung, dass es überflüssig ist, Tieren moralische Rechte zuzusprechen. Moralische Rechte sollten allein Menschen vorbehalten sein, da Träger von Rechten Respekt vorm eigenen Leben und vor dem von Anderen haben müssen. Fehlt dieses Bewusstsein kann kein Anspruch auf Rechte erhoben werden. Tiere sind nicht in der Lage aktive Entscheidungen im Sinne von Interessensabwägungen zu treffen, daher ist es unnötig ihnen Rechte zuzusprechen, die sie aufgrund ihrer Veranlagung niemals nutzen könnten. Tiere können weder Einspruch noch Beschwerde einlegen, das können nur andere für sie übernehmen, die die Belange und das Wohlbefinden der Tiere respektieren und danach handeln (Taylor 2003: 249).

Holand ist der Meinung, dass Lebewesen nur dann moralisch zählen, wenn sie autonom, rational und selbstbewusst sind. All diese Charakteristika treffen auf Tiere nicht zu (Holand 1984). Fox wird an dieser Stelle noch detaillierter. Für das Recht auf moralische Berücksichtigung fordert er kritische Selbstwahrnehmung, die Fähigkeit komplexe Konzepte zu verstehen und anzuwenden, eine kultivierte Sprache, die Fähigkeit zu manipulieren, zu reflektieren, zu planen, etwas zu durchdenken sowie Verantwortung für das eigene Handeln zu übernehmen, einen Lebensplan zu gestalten und sich selbst zu verwirklichen (Fox 1984; Sztybel 2000).

Narveson (1983) ist der Meinung, dass Tiere aus zwei Gründen keine Rechte haben sollten: Zum einen können Tiere nicht moralisch denken und handeln. Zum anderen können mit ihnen keine Vereinbarungen getroffen werden, selbst wenn dies vom Menschen gewollt ist. In den meisten Fällen werden Vereinbarungen getroffen um daraus beiden Seiten einen Vorteil zu verschaffen. Eine Vereinbarung zwischen Menschen und Tieren, die auf ein Nutzungsverbot von Tieren durch den Menschen abzielt ist einseitig, da der Mensch für sich keine Vorteile aus der Vereinbarung ziehen könnte. Dass wir mit Kindern, Senioren oder Behinderten Vereinbarungen eingehen, obwohl diese ebenfalls einseitig sind und aus diesen meist kein Vorteil entsteht, begründet Narveson durch die Familienzugehörigkeit. Selbst wenn ein Teil 
der Gesellschaft diese Gruppen schlecht behandeln will, gibt es immer jemanden, der sich für ihr Wohlergehen einsetzt. Da bei der Nutzung von Tieren jedoch keine Konsequenzen $\mathrm{zu}$ erwarten sind, wird die Nutzung des Tieres stetig ausgebaut (Sandøe et al. 1997:5).

Peter Carruthers vertritt die Meinung, dass Tiere, egal welcher Entwicklungsstufe, über keinen moralischen Status verfügen und ihnen aus diesem Grund keine Rechte zustehen (Carruthers 2014:219). Tieren moralisches Denken zuzusprechen ist eine Form des Anthropomorphismus, also eine Vermenschlichung des Tieres (Sztybel 2001:264). Menschen, Säuglinge, Demente sowie geistig und körperlich Behinderte verfügen dagegen über einen moralischen Status (Carruthers 2014:219). Dabei bestreitet Carruthers nicht, dass Tiere leidensfähig sind, Wünsche und Ängste haben sowie Gefühle empfinden. Er zweifelt jedoch an, dass Tiere rational handeln und somit ihr Verhalten an allgemeine Regeln anpassen können.

\subsubsection{Naturbezogene Vernunftethik}

„Ich bin Leben, das leben will, inmitten von Leben, das leben will“ ist die Kernaussage von Albert Schweitzers universellen Ethik „Erfurcht vor dem Leben“ (Balsinger 2007:11). Schweitzer ist der Ansicht, dass Menschen allen Organismen, Tiere und Pflanzen eingeschlossen, ehrfürchtig begegnen müssen. Er ist prinzipiell dafür, dass alle Wesen angemessen berücksichtigt werden müssen. Obwohl Schweitzer der Meinung ist, dass Tiere fühlende Wesen sind und auch als solche behandelt werden sollten, steht ihnen dennoch nicht die gleiche Behandlung wie Menschen zu (Wibbecke 2013).

„Mit der Notwendigkeit gibt uns die Natur das Recht, niederes Leben dem höheren zu opfern. Aber indem wir davon Gebrauch machen, haben wir uns unsere Verantwortung immer gegenwärtig zu halten. “ (Schweitzer 2006:72).

Er rechtfertigt die Nutzung von Tieren in Laboren mit der Gewinnung von neuen wissenschaftlichen Erkenntnissen, durch welche einer Vielzahl an Lebewesen geholfen werden kann. Das Leid von einigen Wenigen unterliegt demnach deutlich der Verbesserung der Lebensqualität von Vielen. Unter der Berücksichtigung seines 
Leitsatzes „Ehrfurcht vor dem Leben“ postuliert Schweitzer jedoch einen bewussten Umgang mit Tieren. Die Vernunftfähigkeit des Menschen berechtigt diesen, Entscheidungen über Tiere zu treffen. Diese sollten jedoch gut überlegt und ehrfürchtig getroffen werden. Einen unüberlegten und hohen Tierverbrauch lehnt Schweitzer ab (Wibbecke 2013).

\section{Ergebnisse und Diskussion}

Die Nutzung von Tieren zur Erzeugung von Nahrungsmitteln ist ein auf ethischer Ebene intensiv diskutiertes Thema. Ältere Beiträge wie beispielsweise von Aristoteles vertreten den ethischen Standpunkt, dass Tiere nur für die Befriedigung menschlicher Bedürfnisse existieren. René Descartes verbreitete sogar die Meinung, dass Tiere nichts mehr seien als Maschinen, die weder sprechen noch fühlen können und aus diesem Grund auch nicht berücksichtigt werden müssen. Seitdem Singer, Regan und Bentham diese tierethische Diskussion aufgegriffen haben, wird vermehrt über die Leidensfähigkeit des Tieres gesprochen. Tiere sind in der Lage Freude und Leid zu empfinden. Ihre Interessen sollten aus diesem Grund auch stärker berücksichtigt werden.

Rechtlich wurde die Verpflichtung zur Berücksichtigung von Tieren bereits 1933 durch das Inkrafttreten des ersten deutschen Tierschutzgesetzes beschlossen. Seit 2002 ist der ethische Tierschutz als Staatsziel ins deutsche Grundgesetz aufgenommen worden, wodurch dem Tierschutz ein hoher Stellenwert im deutschen Rechts- und Wertesystem zugesprochen wurde. 2010 wurde schließlich im Vertrag über die Arbeitsweise der Europäischen Union eine weitere Weiche zur Verbesserung des Tierschutzes auf EU-Ebene gestellt, indem festgehalten wurde, dass dem Tier in vollem Umfang als fühlendes Wesen Rechnung getragen werden soll. Im zweiten Abschnitt wird Artikel 13 jedoch aufgeweicht, indem „Gepflogenheiten von Mitgliedsstaaten“ berücksichtigt werden. Dieser Abschnitt ist mit der Formulierung nach dem „vernünftigen Grund“ im Tierschutzgesetz gleichzusetzen (vgl. S. 8). Es wird viel Spielraum für Interpretationen und Auslegungen zugelassen, sodass viele Zustände und Verfahren mit der Rechtfertigung einer gesellschaftlichen Tradition begründet werden können. Durch die weiche und teilweise unkonkrete Formulierung geht der eigentliche Zweck, der 
Schutz des Tieres, ein stückweit verloren, da viele Situationen Auslegungssache sind. Als Beispiel ist hier das Töten der männlichen Eintagsküken von Legerassen zu nennen. Bis 2014 wurde dieses Verfahren in allen Bundesländern im Rahmen des Tierschutzgesetzes als „vernünftiger Grund“ akzeptiert, da die männlichen Küken weder für die Produktion von Eiern noch für die von Fleisch verwendet werden können. Aktuell wurde das Schreddern lebendiger männlicher Küken jedoch bereits in vier deutschen Bundesländern verboten.

In der tierethischen Diskussion um die Nutzung von Tieren zu menschlichen Zwecken gibt es zahlreiche unterschiedliche Positionen. Der von Singer mitformulierte Diskriminierungsvorwurf des Speziesismus stützt sich auf das Prinzip der Gleichheit, nach dem eine andersartige Behandlung aufgrund eines Merkmals wie beispielsweise der Hautfarbe oder dem Geschlecht unzulässig ist. In Einklang mit diesem Prinzip ist auch die andersartige Behandlung von Tieren aufgrund ihrer Spezieszugehörigkeit zu hinterfragen (Singer 2002).

Speziesismus ist in unserer Gesellschaft allgegenwärtig. Es ist jedoch erkennbar, dass diese Form des Speziesismus eine andere ist als die, die von Singer beschrieben wurde, da keine klare Trennlinie zwischen Tieren und Menschen gezogen werden kann. Haustiere werden häufig intensiv gepflegt. Sie werden geschoren, tragen Kleidung bei widrigen Wetterbedingungen und erhalten medizinische Pflege. Im Kontrast dazu stehen die Lebensbedingungen landwirtschaftlich genutzter Tiere. Sie werden nach den gesetzlichen Mindestanforderungen gepflegt und versorgt, intensivere Fürsorge wird ihnen jedoch in den meisten Fällen nicht zuteil. An dieser Stelle wird deutlich, dass aktuell eine Form des Speziesismus beim Umgang mit Tieren praktiziert wird (Spencer et al. 2006:22).

Insgesamt betrachtet wird jedoch vielmehr eine Dreiteilung deutlich. An der Spitze steht der Mensch, der ganz klar über allen anderen Lebewesen steht. Darunter folgen Heim- und Haustiere. Ihre Interessen werden zum Teil berücksichtigt, weil dieses im Interesse des Halters steht. Ganz deutlich von den Haustieren abgegrenzt stehen Nutztiere, deren Existenzgrundlage die Nahrungsmittellieferung zu sein scheint. 


\section{Abbildung 2: Dreiteilung von Speziesismus}

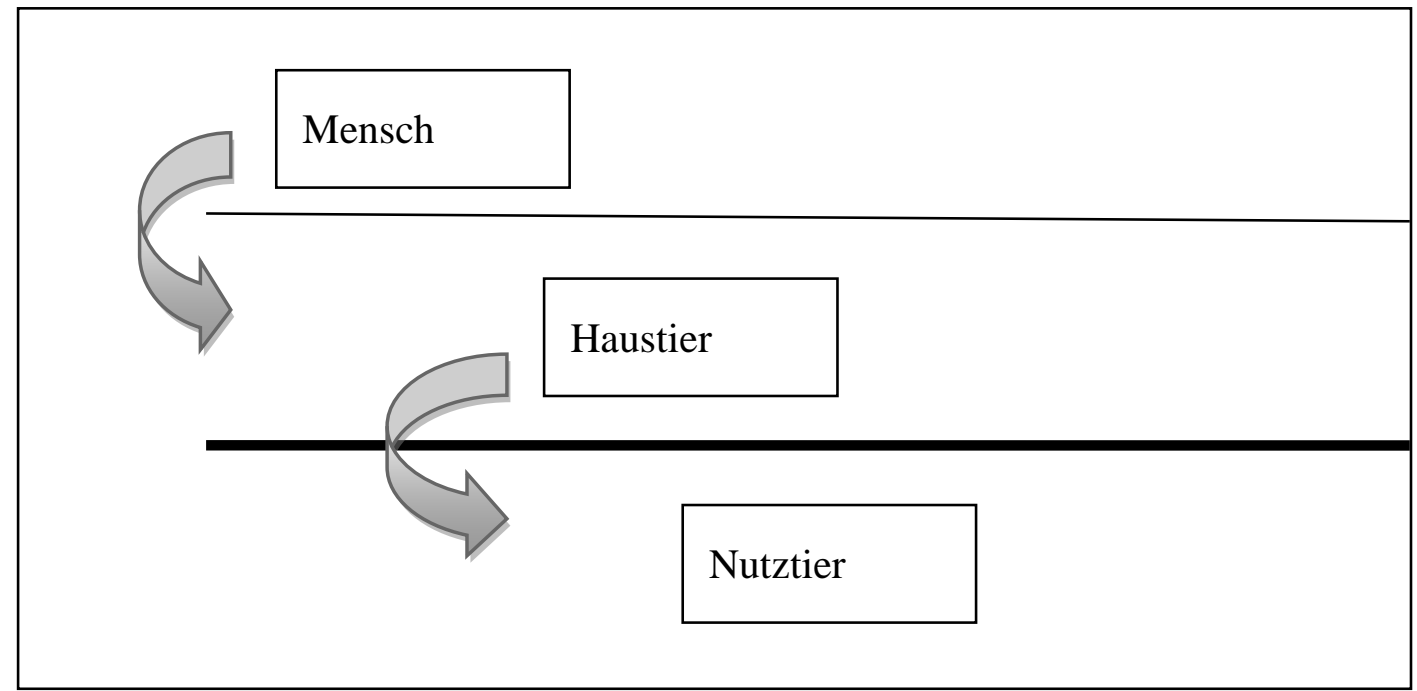

Quelle: Eigene Darstellung

Diese Form des Speziesismus könnte als „Wirtschafts- oder Nutzen- Speziesismus“ bezeichnet werden, da die Abgrenzung nicht nur anhand der Zughörigkeit der Art festgelegt wird, sondern eine weitere Einteilung nach der Wirtschaftlichkeit oder der Nutzungsform des Tieres stattfindet. Ein Pferd war vor der Modernisierung und Technisierung der Landwirtschaft beispielsweise ein wichtiges Arbeitsgerät. Nachdem landwirtschaftliche Nutzfahrzeuge immer erschwinglicher wurden, gab es diesen Verwendungszweck für das Pferd nur noch eingeschränkt. Heute wird es zum einen für sportliche Zwecke oder als Reitpferd und zum anderen nach wie vor zur Fleisch- und Lederproduktion gehalten (Gierse 2002). Es scheint somit immer notwendiger, nicht nur die Spezies zu berücksichtigen sondern vielmehr die Nutzungsform des Tieres.

Utilitaristen zielen nicht nur auf die Befriedigung von Interessen einzelner empfindungsfähiger Wesen ab. Wichtiger ist ihnen die Bedürfnisbefriedigung der Gesamtheit (Doğan 2011:274). Aufbauend auf den fünf Aspekten von Pluhar 1990 ist eine utilitaristische Argumentation ungeeignet um insbesondere die Interessen von Tieren zu schützen. Nach diesen Grundsätzen wäre die moderne Nutztierhaltung im Sinne des Utilitarismus zu rechtfertigen, da die Tiere allein zu dem Zweck der Nahrungsmittelgewinnung gezüchtet werden. Ohne diesen Zweck würden die Tiere 
nicht existieren. Durch die modernen Schlachtungsprozesse wird eine schmerzfreie und schnelle Tötung in den meisten Fällen sichergestellt und auch nahestehende Tiere, wie beispielsweise Nachwuchs, werden weiterversorgt. Die Bedingung ein erfülltes Leben des Tieres zu gewährleisten ist jedoch kritisch zu hinterfragen, da sie nicht allgemein beantwortbar ist. Jedes Individuum hat eigene Ansprüche an ein erfülltes Leben. Ob das Leben für das Individuum erfüllt war, kann für das Tier nicht beantwortet werden, da es sich hierzu nicht äußern kann. Wenn diese Bedingung abgeschwächter formuliert werden würde, sodass lediglich Schmerz-, Angst- und Leidensfreiheit vorausgesetzt werden, dann wären die Kriterien zur Nutzung von Tieren im Sinne des Utilitarismus weniger angreifbar. Bislang ist die Theorie des Utilitarismus auf Seiten gegen die moderne Nutztierhaltung einzugliedern, da die Voraussetzung, dass das Tier ein erfülltes und schmerzfreies Leben hatte, in der modernen Nutztierhaltung nicht erfüllt ist.

Die utilitaristische Theorie scheint aus einem weiteren Grund ungeeignet um ein Nutzungsverbot von Tieren zu erwirken: Bei den aktuellen Fleischverzehrszahlen ist es nicht zu leugnen, dass landwirtschaftliche Nutztiere momentan vorwiegend zur Bedürfnisbefriedigung des Menschen gehalten werden. 2012 wurden in Deutschland 63,1 Millionen Tiere geschlachtet. Demgegenüber stehen abzüglich der $9 \%$ Vegetarier und Veganer 73,3 Millionen Menschen, die Fleisch verzehren. Diese enorme Menge an Schlachtvieh bedeutet im Sinne des Utilitarismus eine signifikante Verschlechterung der Bedürfnisbilanz aller empfindungsfähigen Wesen. Sie bleibt dennoch positiv, da mehr Menschen Fleisch konsumieren als Tiere geschlachtet werden. Erst wenn eine Gewichtung der Bedürfnisse stattfindet, indem dem relativ geringen Bedürfnis nach Fleisch das große Bedürfnis nach Überleben gegenübergestellt wird, verändert sich die Gesamtbilanz zugunsten der Tiere. Würden Menschen ihren Fleischkonsum jedoch derart einschränken, dass auf Seiten der Tiere ein noch geringerer Verlust auftritt als positive Effekte auf Seiten des Menschen, so wäre die Gesamtbilanz erneut positiv. Dadurch wäre, wenn auch eingeschränkt, die Nutzung von Tieren akzeptabel. Eine solche Reduktion des Fleischkonsums wäre sogar denkbar, da der aktuelle Verzehr tierischer Produkte in der menschlichen Ernährung den natürlichen Bedarf übersteigt.

Tierrechtler wie Henry Salt stehen dafür ein, Tieren genau wie Menschen Rechte anzuerkennen, da sie genau wie Menschen über einen Charakter, Vernunft und 
Individualität verfügen. Peter Singer konstatiert, dass es im Kampf um mehr Rechte für Tiere nicht darum geht, diesen die gleichen Rechte wie uns Menschen anzuerkennen. Ein Schwein könne beispielsweise nicht wählen, es ist demnach sinnlos über ein Wahlrecht für das Schwein zu diskutieren, so Singer. Das sei vergleichbar mit der Forderung nach einem Abtreibungsrecht für Männer. Vielmehr geht es in dieser Bewegung um die gleiche Berücksichtigung von Interessen (Singer 1977:15).

Die Nutzung von Tieren durch einen höheren Grad an Rationalität, Intelligenz, Autonomie oder moralischer Sensitivität des Menschen dem Tier gegenüber zu rechtfertigen erscheint angreifbar (Pluhar 1990). Es wird im Tierreich immer wieder Arten geben, die dem Menschen hinsichtlich einiger Fähigkeiten überlegen sind. Die Nase eines Hundes ist beispielsweise um ein Vielfaches feiner als die des Menschen und die Fähigkeit zu fliegen haben uns alle Vögel und Insekten voraus. Dies stellt das in der Tierethik genutzte Grenzfallargument dar. Es beschreibt, dass zwischen Menschen und Tieren keine scharfe Trennlinie gezogen werden kann, da viele Fähigkeiten, wie auch das Schmerzempfinden, nicht exklusiv menschlich sind. Somit können diese auch nicht als Argument für eine andersartige Behandlung verwendet werden. Unter Menschen gibt es genauso wie unter den verschiedenen Tierarten Unterschiede in ihren Fähigkeiten. Es gibt kein Merkmal, das ausnahmslos auf alle Menschen zutrifft. Aus diesem Grund können unterschiedliche Fähigkeiten auch nicht als Begründung für eine ungleiche Interessensberücksichtigung von Menschen und Tieren verwendet werden.

Die Annahme, dass Tiere kein moralisches Empfinden haben gibt nicht gleichzeitig vor, dass Tiere im menschlichen moralischen Verhalten keine Berücksichtigung finden sollten. Als „Moral agents“ obliegt es der Pflicht der Menschen das Glück zu maximieren und Schmerzen und Leid zu minimieren. Es kann nicht davon ausgegangen werden, dass menschliche mentale Erfahrungen die einzigen sind die existieren. Wie viele andere angeborene Fähigkeiten sind jedoch auch diese bei den betroffenen Arten unterschiedlich stark in ihrer Ausprägung und Komplexität (Asquith 2011:240).

Die aktuelle Tierschutzpolitik ist nicht eindeutig in eine der hier dargestellten tierethischen Positionen einzuordnen. Es können anthropozentrische Merkmale 
identifiziert werden, da der Mensch in der deutschen Gesetzgebung im Mittelpunkt steht und hauptsächlich die Interessen des Menschen vertreten und gesichert werden.

In jüngster Zeit wurden jedoch einige tierethische Theorien, die auf der Annahme aufbauen, dass es sich beim Tier um ein empfindungsloses Wesen handelt zunehmend verworfen. Die Analyse von tierethischen Theorien und moraltheoretischen Ansätzen zeigt, dass diese mehrheitlich darin übereinstimmen, dass Tiere als empfindungsfähige Mitgeschöpfe rücksichtsvoll behandelt werden sollten (Ach 2013:36). Diese neuen Erkenntnisse werden zunehmend in die Gesetzgebung intergiert (TschG 2006).

Je lauter die Diskussion um den Tierschutz, die Tierhaltung und Schlachtung werden, desto leiser werden die Argumente, die die Nutzung von Tieren zu menschlichen Zwecken rechtfertigen. Grundvoraussetzung für die Nutzung von Tieren für den menschlichen Zweck ist es, die tierischen Belange unter diejenigen des Menschen zu stellen. In den heutigen Kulturkreisen ist es gesellschaftlich akzeptiert, dass die menschlichen Interessen Priorität haben. Somit ist die Voraussetzung dafür geschaffen, dass Theorien, die die Nutzung von Tieren befürworten, nach wie vor eine große Bedeutung haben.

Ein Kompromiss scheint Schweitzers naturbezogene Vernunftethik zu sein, nach der Tiere zwar für menschliche Zwecke genutzt werden dürfen, dies jedoch ehrfürchtig und überlegt ablaufen sollte. Die Frage, ob Tiere von Menschen genutzt werden dürfen oder ob dies moralisch nicht zu rechtfertigen ist, kann nicht eindeutig und allgemein beantwortet werden. Problematisch scheint zum aktuellen Zeitpunkt vor allem, dass Nutztiere in modernen Tierhaltungssystemen leiden.

\section{Fazit}

Seitdem sich in Europa Lebensmittelskandale häufen, ist vor allem das Vertrauen in das sensible Produkt Fleisch aber auch in andere tierische Produkte geschwächt. Dazu haben nicht nur Krankheiten wie BSE und die Vogelgrippe beigetragen, sondern in jüngster Zeit auch immer häufiger ein ethisch moralisch zweifelhafter Umgang mit Tieren. Auf rechtlicher Ebene werden die Interessen von Tieren zwar zunehmend geschützt, dennoch steht deren Nutzung zur Befriedigung menschlicher Bedürfnisse vor dem Gesetz außer Frage. Unter Tierethikern besteht eine intensive Diskussion um die Nutzung des Tieres zu menschlichen Zwecken. 
Die Analyse verschiedener tierethischer Theorien, die sich mit der Nutzung von Tieren beschäftigen hat ergeben, dass der Begriff des Speziesismus neu gefasst werden muss. Unter Speziesismus wird die Diskriminierung von Individuen aufgrund ihrer Zugehörigkeit zu einer Spezies verstanden. Bei den aus Wildtieren domestizierten Nutz- und Haustieren kann jedoch nicht mehr von einer Speziesdiskriminierung gesprochen werden. Haustiere werden von vielen Tierhaltern im Vergleich zu Nutztieren bevorzugt behandelt. Aus diesem Grund sollte eher von „Wirtschafts-“ oder „Nutzen- Speziesismus“ gesprochen werden, da die Diskriminierung des Tieres nicht nur anhand der Spezieszugehörigkeit erfolgt, sondern auch durch die Nutzungsform.

Des Weiteren wurde herausgearbeitet, dass kein Argument in den hier betrachtenden Theorien ein Verbot der Nutzung des Tieres zu menschlichen Zwecken hinreichend begründen kann. In den meisten tierethischen Theorien wird jedoch der Umstand kritisiert, dass Tiere in den modernen Tierhaltungsbedingungen physisch und psychisch leiden müssen. Diesem Zustand sollte durch eine langfristige Verbesserung der Haltungsbedingungen von Nutztieren entgegengewirkt werden. 


\section{Literaturverzeichnis}

Ach, J., Lüttenberg, B., Grouls, N. 2013: Tiere in der Lebensmittelproduktion: Welche allgemeinen ethischen Schutzkriterien lassen sich begründen?, CfD Drucksache, Münster

Anderson, E. 2014: Tierrechte und die verschiedenen Werte nichtmenschlichen Lebens, in: Tierethik Grundlagentexte, Hrsg:. Schmitz, Suhrkamp Verlag, S. 287- 320

Asquith, P. 2011: Of bonds and boundaries: What is the modern role in Anthropomorphism in primatological studies? American Journal of Primatology, Vol. 73, S. 238- 244

Balsiger, M. 2007: Albert Schweitzers Ethik des Lebendigen „, Leben inmitten von Leben“, Theologischer Verlag Zürich

Baranzke, H. 2002: Würde der Kreatur? Die Idee der Würde im Horizont der Bioethik, Verlag Königshausen und Neumann, Würzburg

Bentham, J. 1780: An introduction to the principles of morals and legislation, in: The Collected Works of Jeremy Bentham, Hrsg.: Burns, J.; Hart, I., Oxford University Press

BMELV 2011: Tierversuchszahlen 2010, URL: http://www.bmel.de/SharedDocs/Downloads/Landwirtschaft/Tier/Tierschutz/2 010-TierversuchszahlenGesamt.pdf?_blob=publicationFile, Abrufdatum 24.10.2014

BMELV 2012: Tierversuchszahlen 2011: http://www.bmelv.de/SharedDocs/Downloads/Landwirtschaft/Tier/Tierschutz/ 2010-TierversuchszahlenGesamt.pdf?_blob=publicationFile, Abrufdatum 24.10.2014

Bundesamt für Veterinärwesen (BVET) 2009: Auslaufvorschriften für Pferde Nr. 11.5

Buschka, S., Rouabma, J. 2013: Hirnloser Affe, blöder Hund? Geist als sozial konstruiertes Unterscheidungsmerkmal, in: Gesellschaft und Tiere- 
soziologische Analysen zu einem ambivalenten Verhältnis, Hrsg.: PfauEffinger, Buschka, Springer Verlag

Carruthers, P. 2014: Warum Tiere moralisch nicht zählen, in: Tierethik Grundlagentexte, Hrsg.: Schmitz, Suhrkamp Verlag, S. 219- 243

Caspar, J. 1998: Anthropozentrismus versus Pathozentrismus - zur Stellung des Tierschutzes im System des grundrechtlichen Freiheitsschutzes, Altex, Vol. 15, S. $205-208$

Caspar, J. 1999: Tierschutz im Recht der modernen Industriegesellschaft. Eine rechtliche Neukonstruktion auf philosophischer und historischer Grundlage, Band 31, Forum Umweltrecht, Nomos Verlagsgesellschaft

Coeckelbergh, M., Gunkel, D. 2014: Facing Animals: A relational, other-oriented approach to moral standing, Journal of Agricultural and Environmental Ethics, Vol. 27, Issue 5, S. 715-733

Deutscher Bundestag 2002: Drucksache 14/8860, Gesetzentwurf der Fraktionen SPD, CDU/CSU, BÜNDNIS 90/DIE GRÜNEN, FDP: Entwurf eines Gesetzes zur Änderung des Grundgesetzes (Staatsziel Tierschutz)

Doğan, A. 2011: A Defense of animal rights, Journal of Agricultural and Environmental Ethics, Vol.24, S. 473-491

Duncan, I. 2005: Science-based assessment of animal welfare: farm animals, Revue Scientifique et Technique de l’Office International des Epizooties, Vol. 24, Issue 2, S. 483-492

Fox, M. 1984: Animal Experimentation: Avoiding Unnecessary suffering, in: National Symposium on Imperatives in Research Animal Use: Scientific Needs and Animal Wellfare, S.112

Gesetz über das Verbandsklagerecht und Mitwirkungsrechte für Tierschutzvereine vom 25. Juni 2013

Gierse, W. 2002: Menschen und Pferde, Eine 5000 Jahre alte Symbiose zwischen zwei Lebewesen, Books on Demand GmbH

Holand, A. 1984: On behalf of a moderate speciesism, The Journal of Applied Philosophie, Vol. 1, Issue 2, S. 281-291 
Horta, O. 2010: What is Speciesism? Journal of Agricultural and Environmental Ethics, Vol. 23, S. 243-266

Kaiser, o.A. 1934: Das Reichstierschutzgesetz, Klinische Wochenschrift, Vol. 13, Issue 21

Kant, I. 1991: Eine Vorlesung über Ethik, Hrsg.: von Gerd, G., Fischer TB Verlag

Kant, I. 2006: The metaphysics of morals (1797), in: Practical Philosophy the Cambridge edition of the works of Immanuel Kant, Cambridge University Press

Konsolidierte Fassung des Vertrages über die Arbeitsweise der Europäischen Union 30.03.2010, Amtsblatt der Europäischen Union

Kunzmann, P., Schmidt, K. 2012: Philosophische Tierethik, in: Das Tier an sich Disziplinübergreifende Perspektiven für neue Wege im wissenschaftsbasierten Tierschutz, Hrsg.: Grimm, H. und Otterstedt, C., S. 37-60 Vandenhoeck und Ruprecht Verlag

Landestierschutzbeauftragte Hessen 2014: Millionenfache Tötung von männlichen Eintagsküken, URL: http://www.tierschutz.hessen.de/irj/Tierschutz_Internet?cid=7785238c544ff29 0727dadd39909dd0b, Abrufdatum:15.10.2014

Lindemann, G., Lüdtke, N., Matsuzaki, H. 2010: Die Stellung des Tieres in der Entwicklung der Tierschutzgesetzgebung in Deutschland, Japan und den USA, Diskussionspapier der Arbeitsgruppe für soziologische Theorie, Carl von Ossietzky Universität Oldenburg

Maehle, A.-H. 1999: Vom rechtlosen Geschöpf zum Träger von Rechten? Die historischen Wurzeln unserer Tierethik im Denken der Aufklärung, in: Tiere ohne Rechte?, Schriftenreihe des Interdisziplinären Zentrums für Ethik, Europa- Universität Viadrina Frankfurt (Oder), S. 1-11

Mahlke, S. 2014. Das Machtverhältnis zwischen Mensch und Tier im Kontext sprachlicher Distanzierungsmechanismen, Anthropozentrismus, Speziesismus und Karnismus in der kritischen Diskusanalyse, Diplomica Verlag

Narveson, J. 1983: Animal rights revisited, in: Ethics and Animals, Hrsg.: Miller, Harlan und Williams, Humana Press, S. 45-60 
Pluhar, E. 1990: Utilitarian killing, replacement and rights, Journal of Agricultural Ethics, Vol. 3, S. 147- 171

Pollack, U. 2007: Die städtische Mensch-Tier-Beziehung - Ambivalenzen, Chancen und Risiken, Berlin Universitätsverlag

Regan, T. 2004: The case of animal rights, University of California Press

Röcklingsberg, H. 2001: Das seufzende Schwein, Harald Fischer Verlag

Sandøe, P., Crisp, R., Holtug, N. 1997: Ethics, in: Animal Welfare, Hrsg.: Appleby, M. Hughes, B., S. 3-17

Sauerberg, A. und Wierzbitza, S. 2013: Das Tierbild in der Agrarökonomie. Eine Diskursanalyse zum Mensch-Tier Verhältnis, in: Gesellschaft und Tieresoziologische Analysen zu einem ambivalenten Verhältnis, Hrsg.: PfauEffinger, Buschka, Springer Verlag

Schweitzer, A. 2006: Ehrfurcht vor den Tieren, Verlag C.H. Beck, München

Sezgin, H. 2014: Artgerecht ist nur die Freiheit, Eine Ethik für Tiere oder warum wir umdenken müssen, Verlag C.H. Beck

Sidgwick, H. 1907: The methods of ethics, 7. Auflage, Hackett Publishing

Singer, P. 1977: Alle Tiere sind gleich, in: Naturethik, Grundtexte der gegenwärtigen Tier- und ökoethischen Diskussion, Hrsg.: Krebs, A., Suhrkamp

Singer, P. 1997: Befreiung der Tiere- Eine neue Ethik zur Behandlung der Tiere, Hirthammer Verlag, München

Singer, P. 2002: Animal Liberation, New York, Harper Collins

Singer, P. 2006: Practical Ethics, Cambridge University Press

Spencer, S., Decypere, E., Aerts, S., De Tavernier, J. 2006: History and ethics of keeping pets: comparison with farm animals, Journal of Agricultural and Environmental Ethics, Vol. 19, S. 17-25

Spiller, A., Schulze, B. 2008: Zukunftsperspektiven der Fleischwirtschaft Verbraucher, Märkte, Geschäftsbeziehungen, Universitätsverlag Göttingen

Statistisches Bundesamt 2013: Land- und Forstwirtschaft Schlachtungen und Fleischerzeugung 4. Vierteljahr und Jahr 2012, Fachserie 3, Reihe 4.2.1, Wiesbaden 
Sztybel, D. 2000: Taking humanism seriously: “Obligatory” Anthropocentrism, Journal of Agricultural and Environmental Ethics, Vol.13, S. 181-203

Sztybel, D. 2001: Animal Rights: Autonomy and Redundancy, Journal of Agricultural and Environmental Ethics, Vol. 14, S. 259-273

Taylor, A. 2003: Animals and ethics, Broadview Press

Tierschutzgesetz (TSchG) in der Fassung der Bekanntmachung vom 18. Mai 2006

Vertrag über die Arbeitsweise der Europäischen Union vom 30.03.2010

Wibbecke, A. 2013: Tierethik, Tierrechte und Moraltheorien, in: Tier- Mensch Pädagogik, , Springer Fachmedien, Wiesbaden, S. 15-27

Wolf, U. 2012: Ethik der Mensch-Tier Beziehung, Klostermann, Frankfurt am Main 


\section{Einsatz von Präferenz- und Motivationstests in der Nutztierhaltung}

\section{-Verbraucherakzeptanz und Zahlungsbereitschaftsanalyse}

\section{Inhaltsverzeichnis}

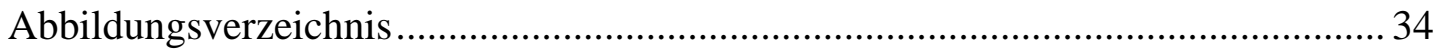

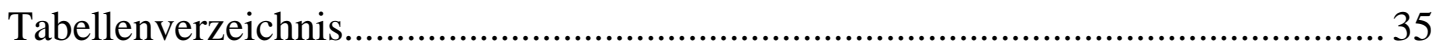

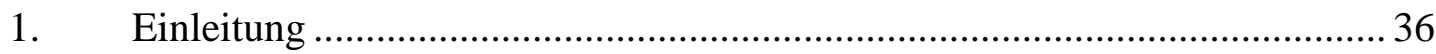

2. Definition tierischen Wohlbefindens ……………………………………........ 37

3. Der Präferenz- und Motivationstest ............................................................... 42

3.1. Hintergrundtheorie zu Präferenz- und Motivationstests .............................. 42

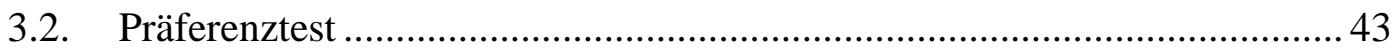

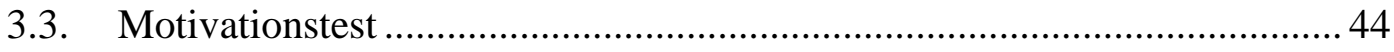

3.4. Motivationstest mit Nerzen ...................................................................... 45

3.5. Kritik an Präferenz- und Motivationstests...................................................... 46

4. Material und Methoden .............................................................................. 48

4.1. Forschungsziel................................................................................... 48

4.2. Fragebogenkonstruktion und Datenerhebung............................................... 49

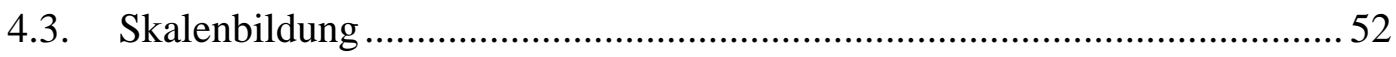

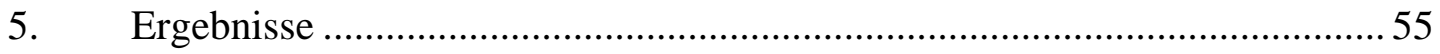

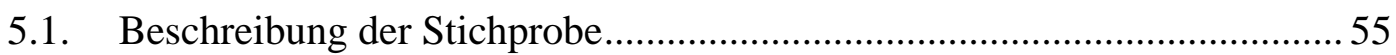

5.2. Bewusstseinszuschreibung von Menschen und Tieren .................................. 56

5.3. Einstellung zur aktuellen Tierhaltung und Fleischkonsum ............................ 58

5.4. Einschätzung und Validität von Präferenz- und Motivationstests ............... 62

5.5. Zahlungs- und Mehrzahlungsbereitschaft für tierische Produkte aus mit Präferenz- und Motivationstests optimierter Haltung ................................................ 63

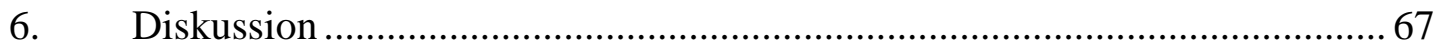

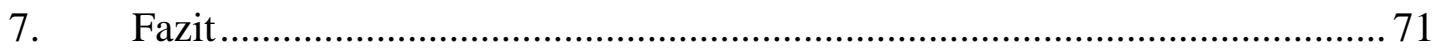




\section{Abbildungsverzeichnis}

Abbildung 1: Definitionsansatz für tierisches Wohlbefinden 40

Abbildung 2: Häufigkeiten der Studienfächer .... .55

Abbildung 3: Streudiagramm der Bewusstseinszuschreibung von Menschen und Tieren 56

Abbildung 4: Schrittweise Regressionsanalyse zur Erklärung des Fleischkonsums . 62 Abbildung 5: Zahlungsbereitschaft für Milch, Eier und Hackfleisch in Abhängigkeit vom Studienfach . .64

Abbildung 6: Mehrzahlungsbereitschaft für 1 l Milch aus mit Präferenz- und Motivationstests optimierter Haltung in Abhängigkeit vom Studienfach 65

Abbildung 7: Mehrzahlungsbereitschaft für 1 l Milch, 6 Eier und 250 g Hackfleisch aus mit Präferenz- und Motivationstests optimierter Haltung in Abhängigkeit vom Studienfach. 66 


\section{Tabellenverzeichnis}

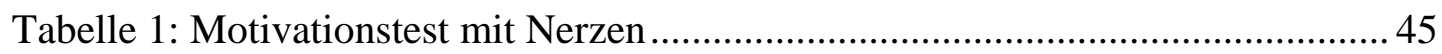

Tabelle 2: Durchschnittliche Produktpreise für Milch, Eier und Hackfleisch ............ 51

Tabelle 3: Reliabilitätsanalyse für die Skalenbildung.............................................. 53

Tabelle 4: Signifikante Mittelwertsunterschiede sowie Effektgröße nach Cohen für die "Bewusstseinszuschreibung Tier" in den Studienfächern................................... 58

Tabelle 5: Fleischkonsum in den Studienfächern .................................................... 59

Tabelle 6: Signifikante Mittelwertsunterschiede sowie Effektgröße nach Cohen für

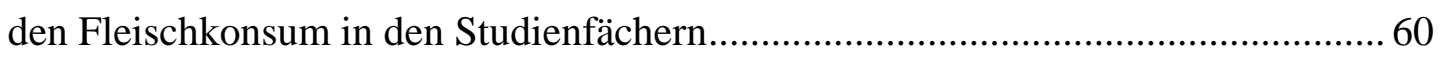

Tabelle 7: Deskriptive Statistik Einschätzung und Validität von Präferenz- und

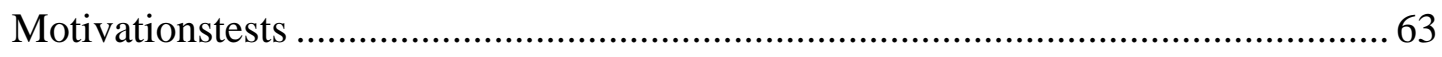

Tabelle 8: Signifikante Mittelwertsunterschiede der Zahlungsbereitschaft für 1 l Milch in Abhängigkeit vom Studienfach .............................................................. 64

Tabelle 9: Marktpreis, Zahlungsbereitschaft und Mehrzahlungsbereitschaft für Milch, Eier und Hackfleisch aus mit Präferenz- und Motivationstests optimierter Haltung. 70 


\section{Einleitung}

Die Lebensmittelindustrie ist in der heutigen Gesellschaft einer der sensibelsten Wirtschaftszweige. Kaum ein anderer Sektor ist von einer solchen Vielzahl an Reglementierungen durchzogen wie dieser. Verordnungen schützen in erster Linie die Produktqualität der tierischen Erzeugnisse. Erst seitdem sich Lebensmittelskandale in Deutschland häufen werden auch die Umstände der tierischen Produktion kontrovers und öffentlich diskutiert (vergl. Meixner et al. 2012). Im Fokus der Überlegungen stehen dabei immer häufiger tierschutzrelevante Aspekte, die nicht nur die Effizienz der Produktion sondern auch eine artgerechte Haltung thematisieren (Miele et al. 2011). Seither traten viele Novellierungen in den Verordnungen zur Nutztierhaltung, wie beispielsweise das Verbot der Anbindehaltung von Pferden, Schweinen und Rindern (Winterhalder 1994) sowie das Verbot der Legebatteriehaltung bei Hennen, in Kraft (BMELV 2012). Zukünftig werden die Haltungsbedingungen von Nutz- und Labortieren eine immer bedeutendere Stellung innerhalb des Produktionsverfahrens einnehmen. Dabei werden nicht nur notwendige Änderungen in Haltung und Umgang relevant, sondern auch Optimierungen, die das Wohlbefinden der Tiere verbessern.

Der steigende Absatz an ökologisch erzeugten Produkten kann als Indiz für das wachsende Bewusstsein des Verbrauchers für die Relevanz tierfreundlicherer Produktionsweisen in der Landwirtschaft verstanden werden. 2005 wurden beispielsweise 12.600 t Schweinefleisch, 300 Mio. Eier und 379.000 t Konsummilch ökologisch produziert. Bis 2010 war die Produktion bereits auf $24.000 \mathrm{t}$ Schweinefleisch, 621 Mio. Eier und $595.300 \mathrm{t}$ Konsummilch angestiegen. Der Absatz der drei Produkte hat sich innerhalb von fünf Jahren somit fast verdoppelt (BÖLW 2012). Das Bundesministerium für Ernährung, Landwirtschaft und Verbraucherschutz hat 2013 eine Studie veröffentlicht, in der unter anderem die Gründe für den Konsum von ökologisch erzeugten Produkten abgefragt wurden. 85 \% der Befragten gaben an, dass eine artgerechtere Tierhaltung für sie für den Kauf von Bio-Produkten mit ausschlaggebend ist (BMELV 2013).

Viele Haltungsvorschriften in der Nutztierhaltung fokussieren sich jedoch auf die Verbesserung der Produktqualität und nicht auf das Wohlbefinden von Tieren. Nur einige wenige Veränderungen, wie beispielswiese das Verbot von schnabelkürzenden Verfahren oder das Verbot der Kälberenthornung, sprechen direkt das Wohlbefinden 
des Tieres an. Die Entscheidungen, in welchem Rahmen Verbesserungen durchgesetzt werden, werden von Experten getroffen, die versuchen tiergerechte Verfahren in der Nutztierhaltung zu entwickeln. Da sich tierische Präferenzen aufgrund ihrer unterschiedlichen Sinneswahrnehmung deutlich von denen des Menschen unterscheiden können (Sandøe 1996), bietet sich die Möglichkeit an, die Präferenzen von Tieren zu unterschiedlichen Haltungssystemen über direkte Befragungen festzustellen. Diese Ergebnisse können dann für eine Verbesserung der Nutztierhaltung verwendet werden, anstatt Experten über die artgerechte Tierhaltung urteilen zu lassen.

\section{Definition tierischen Wohlbefindens}

Um Verbesserungen im Wohlbefinden des Tieres durch die Optimierung von Haltungsbedingungen durchsetzen zu können, muss zunächst definiert werden, aus welchen Aspekten sich tierisches Wohlbefinden zusammensetzt. Durch das britische Brambell Committee wurden 1965 die sogenannten „Five Freedoms“ herausgearbeitet, welche häufig verwendet werden, um Haltungsbedingungen von Nutztieren zu bewerten. Die „Five Freedoms“ setzen sich aus den folgenden Punkten zusammen.

1. "Freedom from hunger, thirst and malnutrition by ready access to fresh water and a diet to maintain full health and vigor.

2. Freedom from discomfort by providing an appropriate environment including shelter and a comfortable resting area.

3. Freedom from pain, injury or disease by prevention or rapid diagnosis and treatment.

4. Freedom to express normal behavior by providing sufficient space, proper facilities and company of the animal's own kind.

5. Freedom from fear and distress by ensuring conditions and treatment which avoid mental suffering. ”(Webster 2005:12).

Die wichtigste Grundlage dieser „Five Freedoms“ ist, dass das Committee die Annahme akzeptiert hat, dass Tiere Schmerz, Leid und Stress erfahren und 
Emotionen wie Wut, Angst, Frustration und Freude empfinden können (Gonyou 1994:2172).

Die Annahme, dass Tiere in ähnlicher Weise wie Menschen Leid und Schmerz empfinden können ist auf einen Analog- oder Homologschluss zurückzuführen. Dieser baut zum einen auf der Ähnlichkeit von höheren Tieren und Menschen in der Sensorik, Motorik und im zentralen Nervensystem auf und zum anderen auf den Erfahrungen, dass Menschen und Tiere auf Schmerzreize ähnlich reagieren (Dawkins 1982; Hassenstein 1993). Nach Wittmann und Schoberger (2010) hängt physisches Wohlbefinden beim Menschen eng mit psychischem, sozialem und kulturellem Wohlbefinden zusammen und ist keinesfalls isoliert $\mathrm{zu}$ betrachten (vgl. auch Schilling, Zeller 2007). Von Fraser et al. wurden 1997 aufbauend auf den Homologschluss, der Definition des menschlichen Wohlbefindens und den „Five Freedoms“ drei Hauptauffassungen in der „Animal Welfare“ Diskussion unterschieden.

1. „Quality of life“ Argumentation: Die Lebensqualität eines Tieres ist davon abhängig, ob es möglich ist, das Tier in Anlehnung an sein natürliches Umfeld zu halten (Carpenter 1980). Der Begriff „Quality of Life“ lässt nach Welmesfelder 2007 darauf schließen, dass tierisches Wohlbefinden mehr beinhaltet als die Abwesenheit von Schmerz und Leid. Er beinhaltet vielmehr die gesamte Verbindung des Tieres mit seiner Umwelt und die Fähigkeit sich dieser Umwelt anzupassen.

2. „Feelings-based“ Argumentation: Ein gutes Leben für Tiere wird durch die Abwesenheit von Leid im Sinne von Schmerz, Angst, Hunger oder weiteren negativen Aspekten definiert (Duncan 1993; Weber, Zárate 2005:480). Die Leidensfähigkeit von Tieren wird von Dawkins $(1988,1997)$ und Sambraus (1991) im Wesentlichen von den Analogien der menschlichen und tierischen Wahrnehmung, der Gehirnstrukturen und des limbischen Systems abgeleitet (Martin 1996). In den letzten Jahren spielen aufgrund von Haltungsbedingungen jedoch auch vermehrt Langweile, Depressionen und Stress eine bedeutende Rolle wenn es um das Wohlbefinden des Tieres geht (Benson, Rollin 2004:40). Es sollte daher nicht nur auf die Abwesenheit von negativen Gefühlen geachtet werden, sondern ebenfalls auf die Anwesenheit von positiven Gefühlen (Duncan 2005:484). 
3. „Functioning-based“ Argumentation: Das tierische Wohlbefinden ist anhand der Funktionalität des Organismus abzulesen, da tierische Gefühle nicht erforscht werden können (Broom 1991). Allein Merkmale wie Gesundheit, Wachstum, normales Verhalten und Entwicklung dienen als Indikatoren für Wohlbefinden, welches nur dann als reduziert angesehen wird, wenn das Tier physiologisch so beeinträchtig wird, dass sein Überleben oder die Reproduktion gefährdet ist (McGlone 1993, Benson, Rollin 2004). Dieser stark reduktionistische Ansatz findet sich häufig in praxisorientierter Literatur. Er konzentriert sich eher auf Leistung und Rentabilität der Produktion (Weber, Zárate 2005:477). Der große Vorteil dieser Argumentation ist, dass die zugrundeliegenden Faktoren, ganz im Gegensatz zu Gefühlen, messbar und einfach zu erfassen sind (Duncan 2005:484).

Welche der Auffassungen wann greift und welche Faktoren integriert werden hängt nach Fraser (1995) stark von der individuellen oder kulturellen Meinung, der ethischen Position und den dahinterliegenden Wertvorstellungen ab. Keine der hier dargestellten Argumentationsgrundlagen ist ausreichend, um tierisches Wohlbefinden hinreichend zu erfassen, da dieses aus einem Zusammenspiel aller genannten Aspekte resultiert. Artgerechte Haltung und tierisches Wohlbefinden sind demnach multidimensional zu erfassen. Dawkins und Duncan liefern folgende Definition für tierisches Wohlbefinden:

„We suggested, that a broad working description of 'welfare' would include the notions of the animal in complete mental and physical health, the animal in harmony with its environment, the animal being able to adapt without suffering to an artificial environment provided by human beings, and that somehow the animals feelings should be taken into account.” (Duncan 2005:484).

Die Autoren kamen jedoch zu dem Schluss, dass es nicht möglich ist, eine allgemein gültige Definition des Begriffes zu liefern, da es viele Situationen gibt, in denen das Tier physiologische Stressreaktionen zeigt, ohne dass das Wohlbefinden auf den ersten Anschein beeinflusst wird. Duncan verweist an dieser Stelle auf das hohe Stressniveau eines Hengstes beim Umwerben einer Stute kurz vor dem Deckakt. 
Obwohl deutliche Stresssymptome gezeigt werden ist es unklar, ob dies bereits zu vermindertem Wohlbefinden des Tieres führt (ibid:484). Der Gedanke der „ethologischen Bedürfnisse“ ist in der Forschung zum tierischen Wohlbefinden immer präsenter geworden. Ziel ist es, anhand von Verhaltensmustern Rückschlüsse auf das Wohlbefinden von Tieren zu ziehen (Dawkins 1983:1195).

Fraser et al. haben versucht tierisches Wohlbefinden multidimensionaler zu erfassen und zu definieren, indem sie die ethologischen Bedürfnisse der Tiere stärker berücksichtigt haben.

\section{Abbildung 1: Definitionsansatz für tierisches Wohlbefinden}

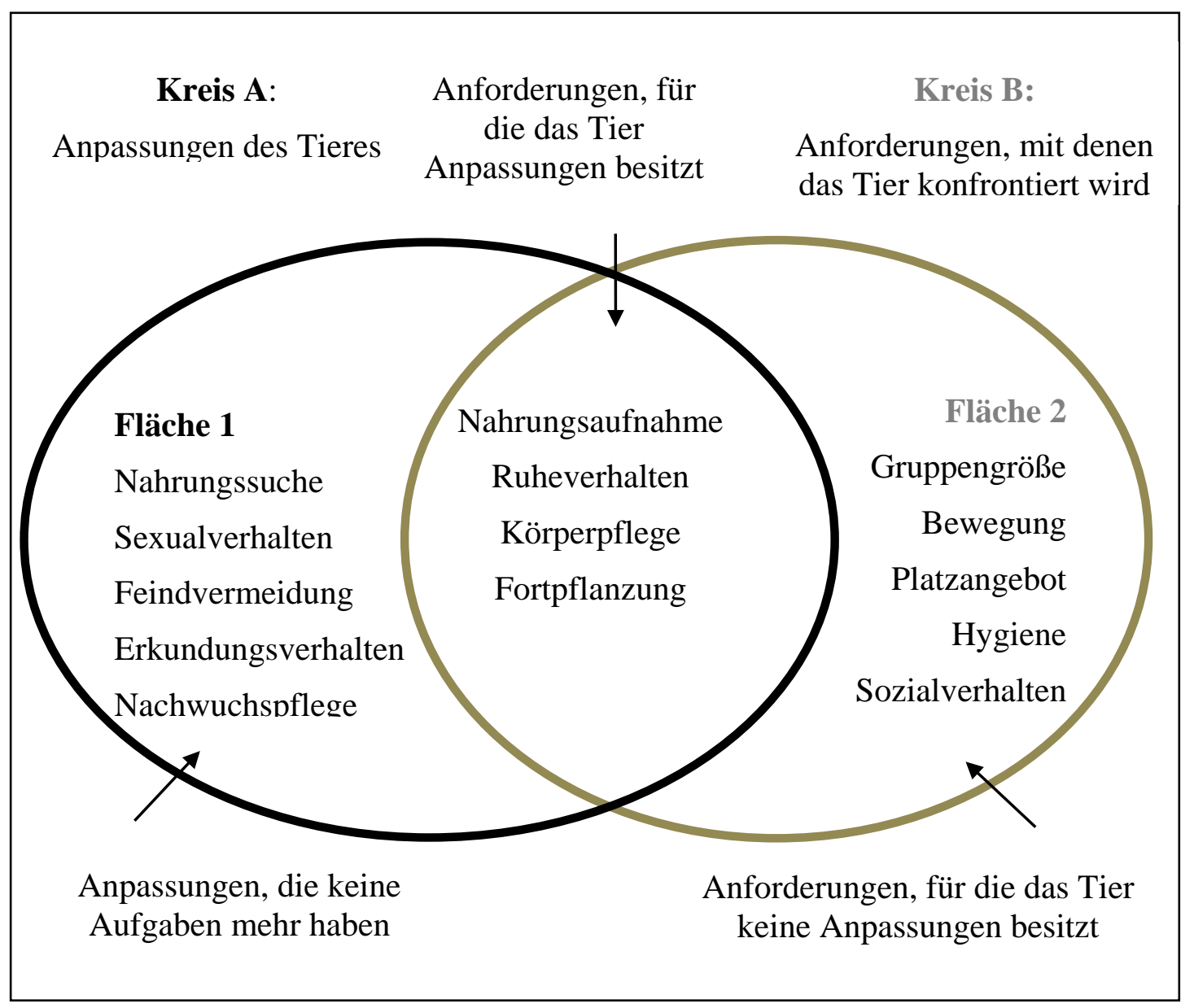


Im Definitionsansatz von Fraser et al. werden alle ethologischen Anpassungen welche sich das Tier aufgrund von Haltungsbedingungen, Umwelteinflüssen oder sonstigen Erfahrungen aneignen musste sowie jene Anpassungen, die zum artspezifischen Verhalten zählen, durch Kreis A symbolisiert. Kreis B beschreibt alle Aufgaben, mit denen das Tier aufgrund seines Haltungsumfeldes konfrontiert wird. Fläche 1 umfasst Anpassungen, die im aktuellen Umfeld keine Funktion mehr bedienen. Das Sandbaden von Hühnern ist beispielsweise eine Anpassung für die Körperpflege. Fehlt ein Sandbad im Käfig so ist diese Anpassung ohne Funktion, da das Huhn diese Verhaltensweise nicht ausführen kann. Das Tier kann sich nicht optimal seiner Umwelt anpassen, es entsteht Stress (Broom 1993). Fläche 2 repräsentiert Anforderungen, mit denen das Tier durch Haltungsbedingungen konfrontiert wird, für die es jedoch keine natürlichen Bewältigungsmechanismen besitzt. Ein Tier in einer großen Gruppe zu halten, obwohl es in der Natur kleinere Gruppen bevorzugt kann eine solche Anforderung sein. Da das Tier keine Verhaltensmechanismen besitzt, um auf diese Änderung einzugehen wird auch in diesem Fall das Wohlbefinden beeinträchtigt.

Die Schnittmenge beider Kreise stellt die Aufgaben und Umstände dar, mit denen das Tier umgehen kann und für die es Anpassungen besitzt. Dadurch führen mögliche Konfrontationen nicht zu Stress oder Einschränkungen, wodurch das Wohlbefinden des Tieres nicht beeinträchtigt wird. Van Putten (1992) bestätigt diese Theorie mit dem Argument, dass tierisches Wohlbefinden durch eine angemessene Harmonie mit der Umwelt erreicht wird. Demnach muss die Umwelt, der das Tier ausgesetzt ist qualitativ innerhalb der Anpassungsfähigkeit des Tieres liegen (Weber, Zátate 2005:479). Aus diesem Definitionsansatz kann der Schluss gezogen werden, dass das tierische Wohlbefinden in Haltungsbedingungen umso besser ist, je größer die Schnittmenge aus Anforderungen und ethologischen Anpassungen ist.

Neben der Möglichkeit theoretische Konzepte zur Bewertung, Messung und Verbesserung tierischen Wohlbefindens heranzuziehen, können auch Präferenz- und Motivationstests dazu genutzt werden, um das Wohlbefinden von Tieren zu optimieren. 


\section{Der Präferenz- und Motivationstest}

\subsection{Hintergrundtheorie zu Präferenz- und Motivationstests}

In der ökonomischen Verhaltensforschung über Konsumenten mit einer limitierten Verfügbarkeit von Einkommen spielen drei Kriterien eine besondere Rolle:

Vom Konsumenten wird eine Auswahl an Gütern getroffen, die seine Bedürfnisse sättigen sollen (Präferenzauswahl) (May 2008). Diese Bedürfnisse spiegeln die Wertvorstellungen des Individuums wider. Der Gegenstand der Präferenzentscheidung kann vielfältig sein.

„Jegliches menschliches Verhalten kann als ökonomisches Wahlhandlungsproblem interpretiert werden, unerheblich ob es sich dabei um den Kauf eines Bildbandes über Naturdenkmäler, die Spende für eine Naturschutzorganisation, die Teilnahme an einer Demonstration gegen ein Straßenbauprojekt oder einen Waldspaziergang handelt.“ (Marggraf 2005:64)

In der Ökonomie wird zudem zwischen Markt- und Nicht-Marktgütern unterschieden. Nicht-Marktgüter können in öffentliche Güter (von öffentlicher Hand zur Verfügung gestellt wie beispielsweise Sicherheit, Polizei) und in Umweltgüter (saubere Luft) unterschieden werden. Marktgüter sind hingegen alle Güter, die auf einem Markt gehandelt und erworben werden können. Die Freizeit, oder die Zeit eines Individuums allgemein, ist als Marktgut zu definieren. Das Individuum hat die Möglichkeit, je nach seinen Präferenzen die vorhandene Zeit mit Arbeit oder Freizeit zu füllen. Fällt die Entscheidung zugunsten von Freizeit aus, so kostet jede Stunde Freizeit, die das Individuum mehr nachfragt den Lohn von einer Arbeitsstunde, die dadurch weniger nachgefragt wird (ibid:64).

Der Konsument kann jedoch nicht frei zwischen allen Gütern wählen. Ein limitierender Faktor (beispielsweise das Einkommen) hindert das Individuum daran, alle Güter seiner Auswahl zu beziehen (May 2008; Marggraf 2005).

Der Konsument wählt die Güterkombination, die ihm den größten Nutzen verschafft (Nutzenmaximierer) (May 2008:19). Dabei betrachtet das Individuum alle Vor- und 
Nachteile sowie Kosten und Nutzen der Alternativen. Die Güterkombination, die die Präferenzen am besten befriedigt und damit den höchsten Nettonutzen liefert wird ausgewählt. Die Auswahl der Güter erfolgt immer unter unvollständiger Information und kann daher bei veränderter Sachlage anders ausfallen (Marggraf 2005).

Steigt beispielsweise der Preis für ein nachgefragtes Gut, so verzichtet der Konsument auf das Güterbündel, auf das er am leichtesten verzichten kann. Die Nachfrage nach diesem Gut ist dann elastisch. Die Güter, die den größten Wert für den Konsumenten haben und auch bei einer Preissteigerung noch bezogen werden, sind in ihrer Nachfrage unelastisch (Paschke 2004:26). Zusammenfassend kann der Schluss gezogen werden, dass Güter mit einer elastischen Nachfrage Luxusgüter und mit einer unelastischen Nachfrage notwendige Güter darstellen (Heertje, Wenzel 2002:141). Von diesen drei Grundbausteinen der Nachfragetheorie ausgehend können Parallelen zum Verhalten von Tieren gezogen werden (Dawkins 1983; Tullock 1971).

\subsection{Präferenztest}

Die Methodik des Präferenztests wurde durch Barry Hughes und Marian Dawkins entwickelt. Der Grundgedanke ist, dass das Tier bei der Auswahl zwischen mehreren Optionen (Präferenzauswahl) immer die Alternative wählt, die für das eigene Wohlbefinden am besten ist (Duncan 2005:486). Das Verhalten des Tieres entsteht daraus, dass es eine bestimmte Handlung ausführt und dadurch eine andere nicht ausgeführt wird. Es wählt demnach zwischen verschiedenen Verhaltensmustern und übt diejenigen aus, welche situativ zum größten Nutzen führen. Das Verhalten des Tieres ist vergleichbar mit dem des nutzenmaximierenden menschlichen Konsumenten. Das tierische Äquivalent zum limitierenden Faktor Einkommen ist die ihm zur Verfügung stehende Körperenergie (Dawkins 1983). Wählt ein Tier eine Handlung bevorzugt vor einer anderen aus, so kann die Annahme getroffen werden, dass die ausgeübte Handlung beim Tier zu einem größeren Nutzen geführt hat als die mögliche Ausübung der Handlungsalternative. Durch Präferenztests kann somit gezielt analysiert werden, welches Verhalten von welchem Tier in spezifischen Situationen bevorzugt wird. 
Unter einem Präferenztest wird die Möglichkeit verstanden, durch gezielte Versuchsanordnungen Handlungspräferenzen von Tieren zu ermitteln. Um herauszufinden, ob ein Tier beispielsweise eine bestimmte Präferenz für eine Einrichtungsalternative zeigt, werden mehrere Räume mit verschiedenen Einrichtungsgegenständen präpariert. Das Tier findet in einem Raum ein Laufrad, in den anderen Räumen einen Kratzbaum oder einen höher gelegenen Sitzplatz vor. Präferenztests laufen ohne Futterangebot ab, da dies die Ergebnisse stark beeinflussen würde. Zu Beginn des Tests wird das Tier wiederholt durch ein Leitsystem zu einer Kreuzung geführt, an der es sich zunächst frei für einen der präparierten Räume entscheiden kann. Ist das Tier mit allen Optionen vertraut, kann die Anzahl der Besuche in den unterschiedlichen Räumen für die Interpretation der Präferenz genutzt werden (Boe et al. 2011; Abou-Ismail, Mahboub 2010). Bei Präferenztests kann die dem Nutztier zur Verfügung stehende Zeit im Sinne der Angebots- und Nachfragetheorie als 'Einkommen' gewertet werden. Verbringt es $80 \%$ seiner Zeit bei einer der Einrichtungsalternativen, dann ist ihm diese Wahl $80 \%$ seines „Einkommens“ wert.

\subsection{Motivationstest}

Der Motivationstest ist ähnlich strukturiert wie der Präferenztest. Neben der Präferenz soll jedoch zusätzlich erfasst werden, wie hoch die Motivation des Tieres ist, an einen bestimmten Einrichtungsgegenstand zu gelangen. Dazu werden Türen vor die Räume montiert, welche sukzessive mit Gewichten beschwert werden. Anhand der Bereitwilligkeit des Tieres dieses Hindernis zu überwinden und dabei Körperenergie einzusetzen kann ermittelt werden, welche „Zahlungsbereitschaft“ das Tier für die jeweiligen Einrichtungsoptionen zeigt. Je höher die aufzuwendende Kraft desto höher ist die Zahlungsbereitschaft des Tieres. Ist das Tier nicht bereit viel Energie einzusetzen um in den Raum zu gelangen, so ist die Nachfrage zu diesem Objekt elastisch.

Als Alternative zu den Gewichten kann auch ein für das Tier unangenehmer Bodenbelag, ein abschreckendes Geräusch oder ein zu überquerendes Wasserbecken eingesetzt werden um die Motivation des Tieres zu messen (Seaman et al. 2008; Koistinen et al. 2008; Manser et al. 1998; Sherwin, Nicol 1996; Bubier 1996). Zur 
Absicherung der Ergebnisse kann der Zugang zu einem Raum verriegelt werden. Diese Verweigerung einer Ressource löst bei dem Tier Stress aus. Der Körper des Tieres schüttet Cortisol aus, welches im Urin nachgewiesen werden kann. Je mehr Cortisol das Tier ausschüttet, desto mehr Stress bereitet ihm das Versperren der Ressource (Mason et al. 2001).

\subsection{Motivationstest mit Nerzen}

Ein solcher Motivationstest wurde mit Nerzen durchgeführt. Nerze werden zur Gewinnung von Pelz in einfachen Drahtkäfigen häufig ohne beschäftigende Ausstattung untergebracht. Dem Deutschen Tierschutzbund zufolge werden hierzulande derzeit ca. 200.000 Nerze auf Pelztierfarmen gehalten (Deutscher Tierschutzbund 2013). Den Nerzen wurden im Präferenztest sieben Räume mit unterschiedlichen Ressourcen zur Wahl gestellt. Die Zugangstüren zu diesen Ressourcen wurden mit Gewichten beschwert, sodass die Tiere einen immer höheren Preis zur Erreichung der gewünschten Ressource zahlen mussten.

Tabelle 1: Motivationstest mit Nerzen

\begin{tabular}{lllll} 
Ressource & $\begin{array}{l}\text { Nachfrage- } \\
\text { elastizität }\end{array}$ & $\begin{array}{c}\text { Konsumenten- } \\
\text { rente (kg) }\end{array}$ & $\begin{array}{c}\text { Anzahl } \\
\text { Besuche }\end{array}$ & $\begin{array}{c}\text { Reservations- } \\
\text { preis (kg) }\end{array}$ \\
\hline \hline Schwimmbecken & $0,26 \pm 0,04$ & $81,41 \pm 9,97$ & 24,00 & $1,25 \pm 0,00$ \\
Alternatives Nest & $0,41 \pm 0,08$ & $60,72 \pm 5,67$ & 22,75 & $1,17 \pm 0,06$ \\
Neue Objekte & $0,58 \pm 0,08$ & $54,58 \pm 5,02$ & 22,50 & $1,16 \pm 0,04$ \\
Plattform & $0,57 \pm 0,07$ & $50,87 \pm 7,65$ & 22,25 & $1,14 \pm 0,06$ \\
Spielzeug & $0,62 \pm 0,05$ & $24,30 \pm 3,25$ & 21,00 & $1,06 \pm 0,07$ \\
Tunnel & $0,73 \pm 0,07$ & $21,61 \pm 1,72$ & 20,75 & $1,06 \pm 0,06$ \\
Mehr Platz & $0,77 \pm 0,06$ & $9,19 \pm 0,90$ & 17,00 & $0,84 \pm 0,07$ \\
\hline \hline
\end{tabular}

Quelle: Mason et al. 2000

Eine geringe Nachfrageelastizität zeigt am Beispiel des Schwimmbeckens, dass die Nerze die Ressource trotz steigendem Gewicht kontinuierlich aufgesucht haben. Demnach erlaubt diese Ressource den Nerzen optimal ihr natürliches Verhalten auszuüben, da für diese die geringste Preiselastizität der Nachfrage sowie die höchste 
Zahlungsbereitschaft ermittelt werden konnte. Für keine weitere Ressource wurde der Höchstpreis von 1,25 kg von den Tieren eingesetzt. Je elastischer die Nachfrage für eine Ressource ist, desto geringer ist die Zahlungsbereitschaft, die Häufigkeit der Besuche sowie der maximale Preis, der zum Erreichen der Ressource gezahlt wird. Zusammenfassend kann aus den Ergebnissen dieses Motivationstests darauf geschlossen werden, dass der Zugang zu Wasser sowie ein alternatives Nest für den Nerz notwendige Güter sind, mehr Platz und ein Tunnel hingegen Luxusgüter, da die Zahlungsbereitschaft hier geringer und die Nachfrage bei steigendem Preis elastisch war.

\subsection{Kritik an Präferenz- und Motivationstests}

Der am häufigsten kritisierte Punkt an Präferenz- und Motivationstests ist, dass adulte Tiere durch ihre bisherige Haltungsform stark beeinflusst werden. Je nach dem wie das Tier aufgezogen wurde, erzeugt Käfig- oder Freilandhaltung besonderen Stress, da die Umgebung für das Tier unbekannt ist. Ein Tier welches in einem Käfig aufgewachsen ist, wird eine starke Präferenz für ähnliche Raumstrukturen aufweisen, da diese ihm vertraut sind und so zu weniger Stress führen. Um stabilere Ergebnisse zu erzeugen wäre es ratsam, die Tiere in Anlehnung an das spätere Versuchsdesign zu halten. Dadurch sind sie bereits an das Umfeld gewöhnt und können sich auf die Einrichtungsalternativen konzentrieren. Zudem muss sichergestellt werden, dass alle Tiere, die für diesen Test eingesetzt werden, ähnliche Vorerfahrungen hinsichtlich ihrer Haltungsbedingungen haben, da das Ergebnis ansonsten stark verzerrt wird (Duncan 2005:486).

Ein weiterer Kritikpunkt ist, dass Präferenz- und Motivationstests sehr eingeschränkt durchführbar sind. Dem Nutztier können immer nur eine Reihe von Alternativen vorgestellt werden, zwischen denen es sich entscheiden muss. Diese situativ ausgewählten Haltungseinrichtungen müssen nicht per se das Wohlbefinden des Tieres positiv beeinflussen. Die Ergebnisse sind lediglich so zu deuten, dass die getroffene Auswahl unter allen vorgestellten Alternativen die bevorzugte ist. Wird einem Gast beispielsweise angeboten, zwischen einem Tee und einem Kaffee zu wählen, dann wird er sich für eine Alternative entscheiden müssen. Es kann jedoch auch sein, dass ein Glas Wasser zu einer besseren Bedürfnisbefriedigung geführt 
hätte als Kaffee oder Tee (ibid). Im Versuchsaufbau kann dies gelöst werden, indem dem Tier so viele Alternativen wie möglich vorgestellt werden, zwischen denen es wählen soll. Unter Umständen wird dadurch jedoch die Auswertung erschwert, da es die verfügbare Zeit in vielen verschiedenen Räumen verbringen kann und so keine klaren Präferenzen ersichtlich werden, wenn es mehrere für das Tier gute Alternativen geben sollte. Des Weiteren kann es vorkommen, dass alle zur Wahl gestellten Alternativen das Wohlbefinden negativ beeinflussen und das Tier lediglich jene Option wählt, die situativ am wenigsten belastend erscheint (ibid).

Dawkins identifiziert ein weiteres Problem. Bei Präferenz- und Motivationstests kann nicht unterschieden werden, ob das Wohlbefinden durch die Auswahl des Tieres nur kurzzeitig oder langfristig positiv beeinflusst wird. Es ist demnach davon abzuraten, aus den Ergebnissen von Präferenztests allgemeingültige und voreilige Schlüsse zu ziehen, da es sich bei den Beobachtungen um Kurz- oder Langzeitpräferenzen handeln kann. Äußere sowie innere Eindrücke und Gegebenheiten beeinflussen die Präferenz des Tieres stark (Dawkins 1982:83). Die durch das Tier ausgewählte Alternative ist zunächst nur so zu deuten, dass diese im seinem jetzigen Gefühls- und Daseinszustand die beste Wahl ist. Beispielhaft dient ein durchgeführter Test mit Broilern. Durch die starke züchterische Selektion haben Broiler ein ausgeprägtes Hungergefühl. Wird ihnen Futter ad libidum zur Verfügung gestellt, so fressen die Tiere so viel, dass ihr Wohlbefinden durch die hohe Gewichtszunahme langfristig nachhaltig negativ beeinträchtigt wird ${ }^{6}$ (Renema, Robinson 2004). Die Dosierung des Futters ist aus diesem Grund langfristig besser für die Gesunderhaltung des Tieres. Bei der Auswertung der Ergebnisse müssen demnach die Kenntnisse über Tierhaltung und -ernährung berücksichtigt werden um langfristig gesundheitliche Schäden am Tier zu vermeiden (Duncan 2005:486).

Trotzt dieser Kritikpunkte sind Präferenz- und Motivationstests dazu geeignet um die Präferenzen von Tieren zu verschiedenen Haltungseinrichtungen zu ermitteln und ihre Motivation festzustellen. Dies zeigen erste Ergebnisse aus Nutztierhaltungsversuchen mit Hilfe von Präferenz- und Motivationstests (Boe et al. 2011; Abou-Ismail, Mahboub 2010; Weber, Zárate 2003; Bessei et al. 2001).

\footnotetext{
${ }^{6}$ Es handelt sich zwar um ein züchterisches Problem, da die Tiere auf ein ausgeprägtes Hungergefühl selektiert wurden. In der modernen Nutztierhaltung wird jedoch in den seltensten Fällen mit ursprünglichen Rassen gearbeitet. Aus diesem Grund ist das Beispiel an dieser Stelle passend.
} 


\section{Material und Methoden}

\subsection{Forschungsziel}

Im aktuellen gesellschaftspolitischen Diskurs werden die Belange des Tieres durch den Menschen vertreten. Es liegt im Ermessen des allgemeinen menschlichen moralischen Empfindens wie mit Tieren umgegangen wird und unter welchen Haltungsbedingungen sie leben. Allein die rechtlichen Rahmenbedingungen wie beispielsweise das Tierschutzgesetz (TierSchG 2005) oder die Nutztierhaltungsverordnung (TierSchNutztV 2006) bieten Anhaltspunkte für ein Mindestmaß an moralischem und ethischen Verhalten im Umgang mit Tieren.

Häufig entscheiden ökonomische und monetäre Argumente über die Grundstruktur der modernen Nutztierhaltung. Um in Zukunft die Nutztierhaltung maßgeblich zu verbessern ist es notwendig, dass Tieren grundsätzlich ein Mindestmaß an Bewusstsein und ethischer Berücksichtigung zugeschrieben wird.

Derzeit ist unklar, wie Konsumenten von tierischen Produkten das Bewusstsein von Tieren einschätzen. Diese werden in der landwirtschaftlichen Produktion häufig unter Mindestanforderungen gehalten. Es wird postuliert, dass Tieren im Vergleich zum Menschen weniger Bewusstsein zugeschrieben wird. Wenn Tieren ein hoher Bewusstseinszustand zugeschrieben wird, dann ist den Probanden die artgerechte Tierhaltung und das empfundene Wohlbefinden des Tieres wichtig. Zudem wird erwartet, dass die Einschätzung des tierischen Bewusstseins, die Zufriedenheit mit dem aktuellen Stand der landwirtschaftlichen Nutztierhaltung sowie die individuelle Relevanz der artgerechten Tierhaltung Einfluss auf den Fleischkonsum haben, da vielen Konsumenten die Umstände der Produkterzeugung wichtig sind und sie bei mangelnder Zufriedenstellung weniger konsumieren.

Um zukünftig das Wohlbefinden eines Tieres stärker zu berücksichtigen und auch um die Wertschätzung tierischen Lebens wieder zu verbessern, könnten Ergebnisse aus aktuellen Präferenz- und Motivationstests mit in Entscheidungsverfahren integriert werden. Anhand der zuvor beschriebenen Ergebnisse von Präferenz- und Motivationstests kann die Überlegung getroffen werden, ob Tiere in Entscheidungsprozesse zu Haltungsfragen integriert werden können, da ihre spezifischen Präferenzen gezielt ermittelt werden können. Wenn akzeptiert wird, dass das Wohlbefinden von Tieren mehrdimensional ist und Abweichungen zu einer 
Beeinträchtigung ihrer Lebensqualität führen sowie dass Tiere ein Präferenzverhalten haben und dieses auch ausdrücken können, so verbleibt die Frage, wie Verbraucher Präferenz- und Motivationstests aufnehmen und einschätzen und ob eine Zahlungsbereitschaft für solche Produkte besteht. Es ist besonders wichtig diese Zahlungsbereitschaft zu ermitteln, da bei einer praktischen Umsetzung und Einbeziehung der Ergebnisse von Präferenz- und Motivationstests mit höheren Kosten auf Seiten der Erzeuger und der Konsumenten zu rechnen ist. Zudem soll analysiert werden, ob der Studienschwerpunkt der Befragten Einfluss auf die Einstellung zum Einsatz von Präferenz- und Motivationstests zur Verbesserung der Nutztierhaltung ausübt. Zwischen den Fachdisziplinen wird aufgrund des Wissensstands und der Identifikation mit der Thematik Tierhaltung eine unterschiedliche Bewertung des aktuellen Zustands der landwirtschaftlichen Tierhaltung, der Wahrnehmung des Tierleids, der Bewusstseinszuschreibung von Tieren und der wahrgenommenen Validität von Präferenz- und Motivationstests erwartet.

\subsection{Fragebogenkonstruktion und Datenerhebung}

In Anlehnung an die Charakteristika des Homo oeconomicus ${ }^{7}$ wurden Fragen zur Bewusstseinszuschreibung entwickelt, um so Menschen und Tiere als wirtschaftlich handelnde Subjekte vergleichen und in Beziehung setzen zu können. Des Weiteren wurden die Teilnehmer zu ihrer Zufriedenheit mit der konventionellen landwirtschaftlichen Praxis und ihrer Einstellung zur Relevanz des tierischen Wohlbefindens in Haltungsbedingungen befragt.

Um sicherzustellen, dass alle Befragten über Grundkenntnisse zu Präferenz- und Motivationstests verfügen, wurden diese beispielhaft an jeweils zwei Tierarten beschreiben. Es wurde zufällig entschieden ob dem Befragten eine Beschreibung mit Kühen oder Nerzen vorgelegt wurde. Die Befragten konnten dabei auch einen Präferenztest mit Kühen und einen Motivationstest mit Nerzen bekommen. Dadurch sollte sichergestellt werden, dass die Tierart keinen Einfluss auf die Einstellung und Bewertung der Testverfahren hat.

\footnotetext{
${ }^{7}$ Homo oeconomicus. Handelt rational unter Knappheit mit einer klaren Bedürfnishierarchie für die maximale Bedürfnisbefriedigung. Restriktionen schränken ihn ein (Marggraf et al. 2005:63).
} 
Neben der Einschätzung von Präferenz- und Motivationstests wurde die Zahlungsund Mehrzahlungsbereitschaft für die tierischen Produkte Milch, Eier und Hackfleisch abgefragt. Problematisch bei Zahlungsbereitschaftsanalysen ist, dass es sich um einen hypothetischen Markt handelt und keiner der Befragten um eine direkte Zahlung gebeten wird. Aus diesem Grund kann die Zahlungsbereitschaft für ein Produkt von den Probanden überschätzt werden. Ein potentieller Messfehler bei der Zahlungsbereitschaftsanalyse kann verschiedene Ursachen haben. Zum einen können unter anderem Befragungsfehler durch beispielswiese hohe Zustimmungstendenzen, Informationsverzerrungen oder soziale Erwünschtheit auftreten. Dabei wird von den Befragten aufgrund gesellschaftlicher Erwünschtheit eine Zahlungsbereitschaft angegeben, um unter anderem eine moralische Befriedigung durch die Äußerung einer Zahlungsbereitschaft zu erfahren. Zum anderen kann durch vorgegebene Orientierungswerte ein Ankereffekt auftreten, wodurch die Höhe der Zahlungsbereitschaft beeinflusst wird (Bräuer, Suhr 2005:154ff).

Die Produkte Milch und Eier wurden zum einem ausgewählt, da sie häufig auch von Vegetariern konsumiert werden. Als drittes tierisches Produkt wurde Hackfleisch ausgewählt. Bei allen drei Produkten wurde gezielt auf eine Preisangabe verzichtet, da sich die Preise von konventionell und ökologisch erzeugten Produkten zum Teil stark unterscheiden und die Befragten so von ihrer individuellen Zahlungsbereitschaft ausgehen konnten. Zur Erfassung der Mehrzahlungsbereitschaft wurden den Befragten Antwortmöglichkeiten vorgegeben. Sie konnten zwischen einer Mehrzahlungsbereitschaft von $0 €$ bis $1 €$ in Zehnerschritten auswählen. Da sich die Produkte alle in einer Preisspanne von 0,70€ bis 2,80€ positionieren kann davon ausgegangen werden, dass keine Beeinflussung der Mehrzahlungsbereitschaft durch die Antwortmöglichkeiten stattgefunden hat. Zur Interpretation der Ergebnisse werden die aktuellen Marktpreise der drei Produkte gewählt. 
Tabelle 2: Durchschnittliche Produktpreise für Milch, Eier und Hackfleisch

\begin{tabular}{lcc}
\multirow{2}{*}{ Produkt } & \multicolumn{2}{c}{ Preis in $€$} \\
\cline { 2 - 3 } & konventionell & ökologisch \\
\hline \hline 1 I Milch (1,5\%, ultrahocherhitzt) & 0,70 & 1,09 \\
$\mathbf{6}$ Eier (Bodenhaltung) & 0,95 & 1,39 \\
$\mathbf{2 5 0}$ g Hackfleisch (Schwein, Rind) & 2,19 & 2,79 \\
\hline \hline
\end{tabular}

Quelle: Eigene Daten

Nach einem qualitativen und einem quantitativen Pretest mit unabhängigen Personen wurde der Fragebogen erneut überarbeitet und schließlich zur Befragung freigeschaltet. Innerhalb eines Frageblocks wurden die Fragen randomisiert um Folgeeffekte ausschließen zu können. Die Studie richtete sich hauptsächlich an Studenten, Absolventen und Promovenden der Fachrichtungen Agrarwissenschaften, ökologische Agrarwissenschaften, Landschaftsökologie, Biologie, Betriebs- (BWL) und Volkswirtschaftslehre (VWL), Jura, Philosophie sowie Psychologie. Diese Fächer wurden aufgrund ihrer fachlichen Nähe zum Thema Tierhaltung, Fleischproduktion und Landwirtschaft ausgewählt. Grundsätzlich war die Umfrage jedoch für jeden Interessenten offen. So sollte erreicht werden, dass Daten aus verschieden Einflussbereichen auf die landwirtschaftliche Erzeugung mit in die Befragung einflossen. Obwohl diese Stichprobe einer disproportional geschichteten Zufallsstichprobe durch die Schichtung anhand des Studienfachs sehr nahe kommt, kann die vorliegende Stichprobe nicht als solche behandelt werden. Die Schichten unterscheiden sich in vielen Fällen nicht ausschlaggebend. Durch die Veränderungen im Studiensystem und vor allem durch die Umstellung auf Bachelor- und Mastersysteme sind die Studieninhalte zum Teil sehr ähnlich geworden. Zwischen den Fächern kommt es zu erheblichen Überschneidungen der Studienfachinhalte. Daher ist es nicht möglich in jedem Fall von heterogenen Schichten zu sprechen. Beispielhaft sind hier die Studienfächer Agrarwissenschaften und Betriebswirtschaftslehre zu nennen. Obwohl eine Kategorisierung durch das Fach in Schichten möglich wäre, unterscheiden sich die Inhalte der Fächer nicht immer grundlegend. Agrarwissenschaftler können sich durch ihre Schwerpunktwahl früh für eine agrarökonomische Ausbildung entscheiden. So besuchen Agrarwissenschaftsstudenten auch Vorlesungen aus dem Betriebswirtschaftsstudium 
und andersherum. Aus diesem Grund wurde darauf geachtet, dass aus jeder Fachgruppe ungefähr gleich viele Probanden befragt wurden. Dies stellte sich vor allem für die Fachbereiche Betriebs- und Volkswirtschaftslehre sowie Philosophie als schwierig heraus, da sich diese Fachbereiche inhaltlich nicht direkt mit landwirtschaftlicher Nutztierhaltung auseinandersetzen und so das Interesse an der Studie eher gering war. Obwohl die Umfrage gezielt an Orten verteilt wurde, an denen die Fachdisziplinen aktiv sind, mussten die Fächer Betriebs- und Volkswirtschaftslehre zusammengefasst werden, um eine ausreichende Gruppengröße vorweisen zu können. Nach der Angabe zu ihrem Studienfach wurden die Studenten zu ihrer individuellen Identifikation mit ihrem Studienfach befragt. Dazu wurde die vier Item Skala von Doosje et al. (1995) übernommen.

Die Umfrage wurde mit Hilfe von Unipark erstellt. Alle Teilnehmer haben online an der Umfrage teilgenommen. Verbreitet wurde sie hauptsächlich über Verteiler von Fachschaften, Semestern und Konferenzen. Des Weiteren wurden OnlinePlattenformen genutzt, wie beispielsweise das „Schwarze Brett“ von StudIP Göttingen. Der Umfragezeitraum erstreckte sich vom 11.12.2013 bis zum 06.02.2014. Es wurde ein längerer Befragungszeitraum gewählt, da die Weihnachtsfeiertage und der Jahreswechsel in diesen Zeitraum fielen. Die Beendigungsquote des Fragebogens beträgt 45,05 \%. Die gewonnenen Daten wurden unter Verwendung von Predictive Analysis Software (PASW) 22 statistisch ausgewertet.

\subsection{Skalenbildung}

Jede Skala wurde durch mindestens drei Items operationalisiert. Durch eine Reliabilitätsanalyse wurde untersucht, ob die Items zu einer Skala zusammengefasst werden können. Notwendige Voraussetzung dafür ist, dass die Items eine einheitliche Orientierung haben und die inhaltliche Bedeutung von hohen und niedrigen Werten übereinstimmt. Wenn dies nicht der Fall ist, dann müssen einzelne Items umcodiert werden. Zudem müssen sich alle Items sinnvoll zu einer Gesamtskala zusammenfassen lassen (Brosius 2013:823). Fast alle Items des Fragebogens wurden mit Hilfe einer endpunktbenannten Skala von 1 bis 5 beantwortet. Dabei war die Ziffer 1 jeweils mit „Nein, lehne ab“ und die Ziffer 5 mit 
„Ja, stimme zu“ beschriftet. Dadurch lassen sich die Items problemlos zusammenfassen. Der Vorteil einer endpunktbenannten Skala ist, dass diese wie eine Intervallskala behandelt werden kann und somit mehr statistische Verfahren zulässt als die Ordinalskala (Brosius 2013:479). Durch den einheitlichen Wertebereich der Skala kann zudem ausgeschlossen werden, dass ein Item ein anderes dominiert (ibid:823).

Ausschlaggebend für die Reliabilität der Skalen ist Cronbachs Alpha $(\alpha)$. Je stärker die Items, die zusammengefasst werden sollen, miteinander korrelieren, desto höher fällt $\alpha$ aus. Die Anzahl der Items, die für eine Skala herangezogen werden, tragen mit abnehmendem Grenznutzen zur Höhe von Cronbachs Alpha bei (Baur, Fromm 2008). Eine Skala gilt ab einem $\alpha=0,7$ als reliabel und darf somit zusammengefasst werden (Brosius 2013:826).

Tabelle 3: Reliabilitätsanalyse für die Skalenbildung

Items

Skala

Cronbachs

Alpha

Menschen ...

...verfolgen eigene Interessen.

...verhalten sich vernünftig.

...mögen manches und lehnen manches ab.

...haben einen freien Willen.

Bewusstsein

...können sich freuen und leiden.

Mensch

0,705

...haben eine eigene Persönlichkeit.

...können niedrige und hohe Lebensqualität unterscheiden.

Tiere ...

...verfolgen eigene Interessen.

...verhalten sich vernünftig.

...mögen manches und lehnen manches ab.

...haben einen freien Willen.

...können sich freuen und leiden.

Bewusstsein Tier $\quad 0,755$

...haben eine eigene Persönlichkeit.

...können niedrige und hohe Lebensqualität unterscheiden. 


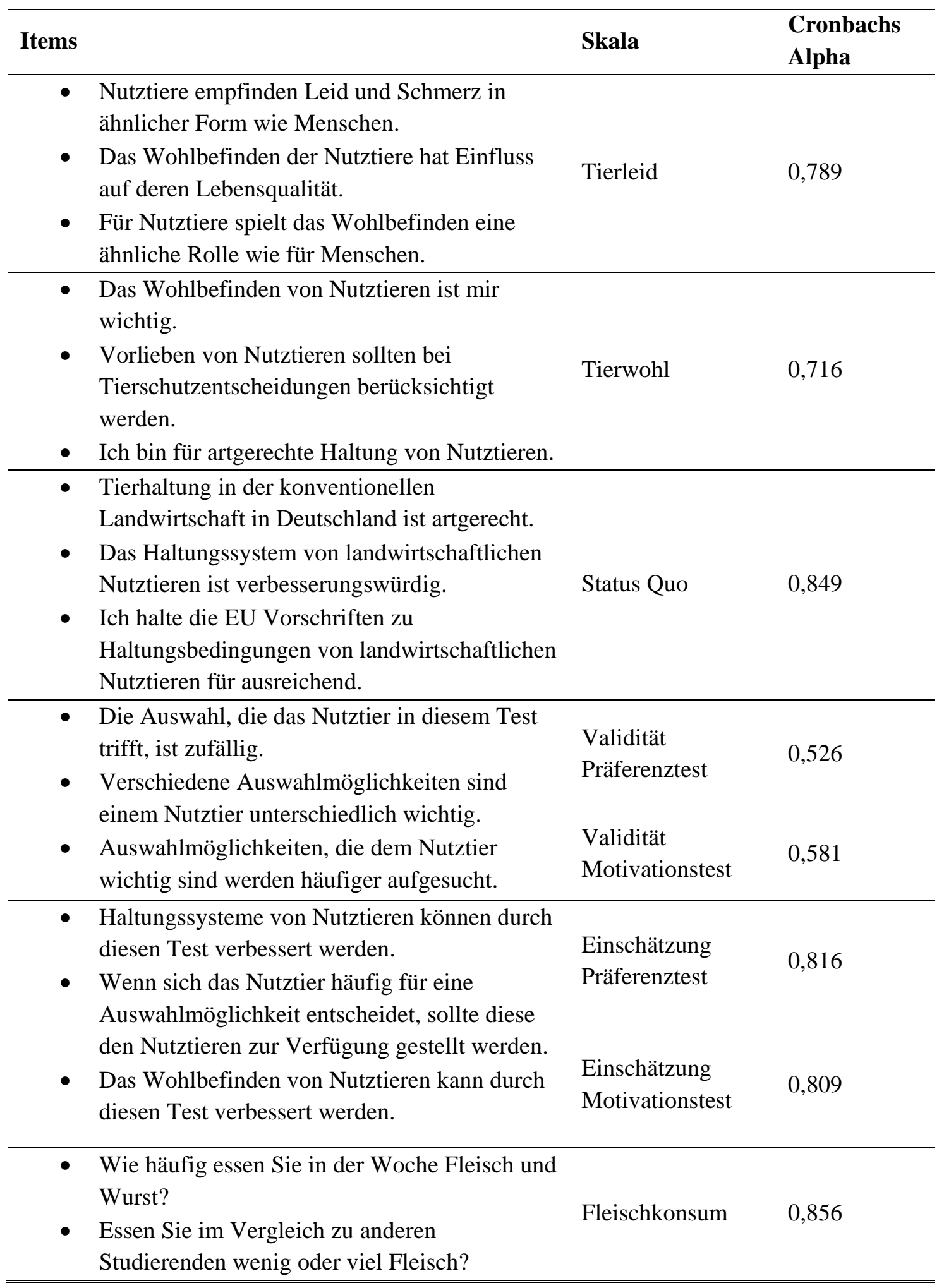

Quelle: Eigene Daten

Die Skalen Validität Präferenztest und Validität Motivationstest liegen mit einem Cronbachs Alpha von 0,526 und 0,581 unter der Grenze von 0,7. Eine höhere Anzahl an Items hätte eventuell zu einer höheren Reliabilität der Skalen führen können. Die 
Skalen Tierleid und Tierwohl korrelieren stark miteinander, aus diesem Grund wurden sie zur Skala Tierleid und -wohl zusammengefasst $(\alpha=0,853)$.

\section{Ergebnisse}

\subsection{Beschreibung der Stichprobe}

Im Rahmen der Befragung zur persönlichen Einstellung und Bewertung von Präferenz- und Motivationstests konnten 619 beantwortete Fragebögen ausgewertet werden. An der Befragung haben 22,9\% männliche und 61,9\% weibliche Studierende teilgenommen, bei 15,2 \% fehlte eine Angabe zum Geschlecht. Damit sind Frauen in dieser Umfrage stärker vertreten als Männer. Deutlich mehr Probanden haben angegeben sich im Bachelor zu befinden als im Master oder der Promotion. Das Durchschnittsalter beträgt 26,28 Jahre (SD=5,44).

Teilnehmer, die mehr als ein Fach studieren, wurden gebeten sich für ein Hauptfach zu entscheiden. Abbildung 2 stellt die Eingliederung der Teilnehmer in die ausgewählten Hauptfächer dar.

\section{Abbildung 2: Häufigkeiten der Studienfächer}

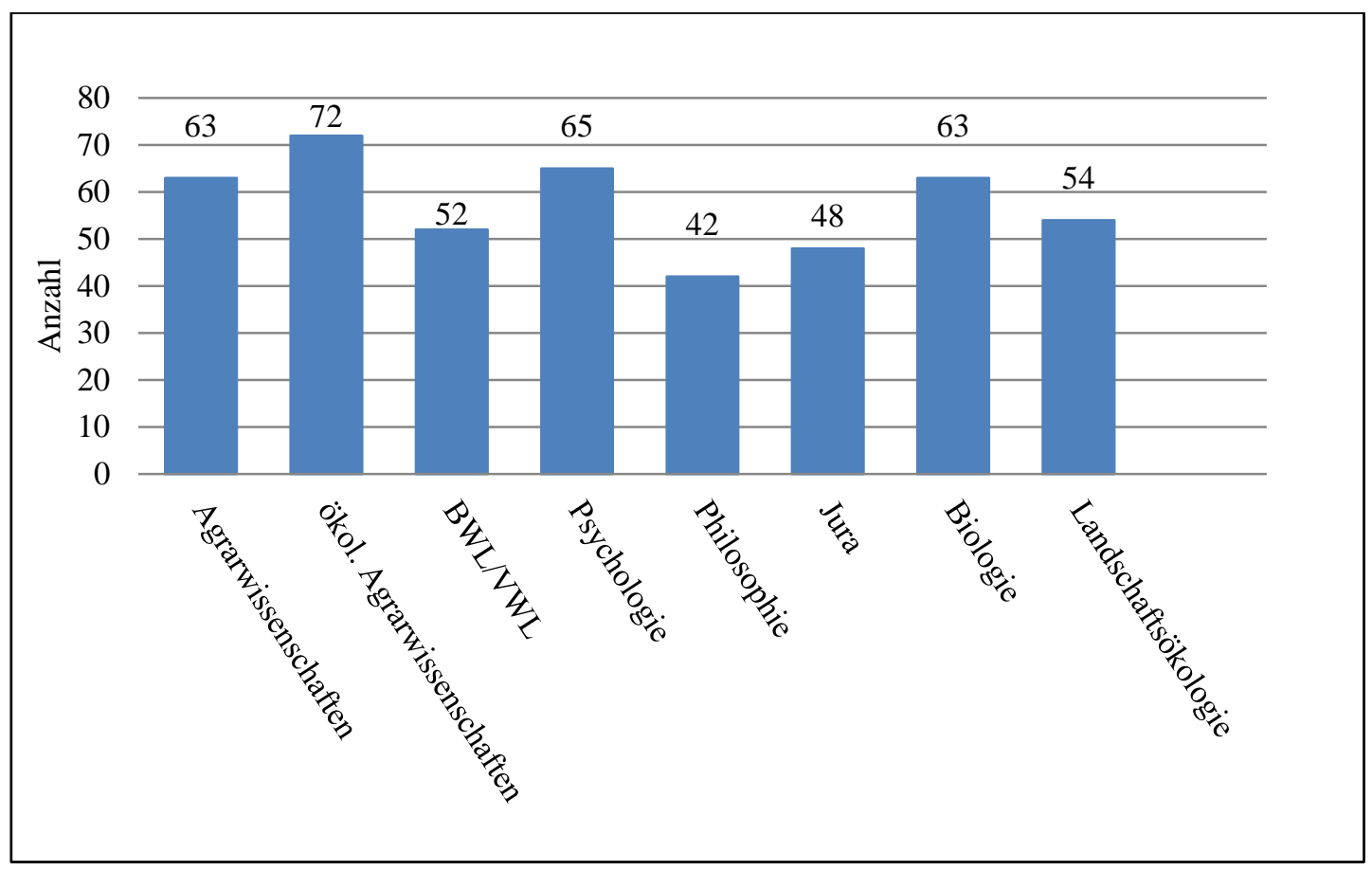

Quelle: Eigene Daten 
Die Anzahl der Befragten aus den Fachdisziplinen ist relativ ausgeglichen. Die

Fachrichtungen Philosophie und Jura bilden die jeweils kleinsten Repräsentantengruppen.

Neben der Fachgruppe wurde ebenfalls abgefragt, wie weit die Befragten in ihrem Studium fortgeschritten sind. 52,2 \% gaben an, sich im Bachelorstudium, Vordiplom oder 1. Staatsexamen zu befinden, 23,1 \% waren im Masterstudium, Diplom oder im 2. Staatsexamen. 7,6 \% hatten bereits ihren Master abgeschlossen und waren im Beruf oder mit ihrer Promotion beschäftigt.

\subsection{Bewusstseinszuschreibung von Menschen und Tieren}

Zum Einstieg in die Thematik wurden die Teilnehmer befragt, wie sie das Bewusstsein von Menschen und Tieren einschätzen.

Abbildung 3: Streudiagramm der Bewusstseinszuschreibung von Menschen und Tieren

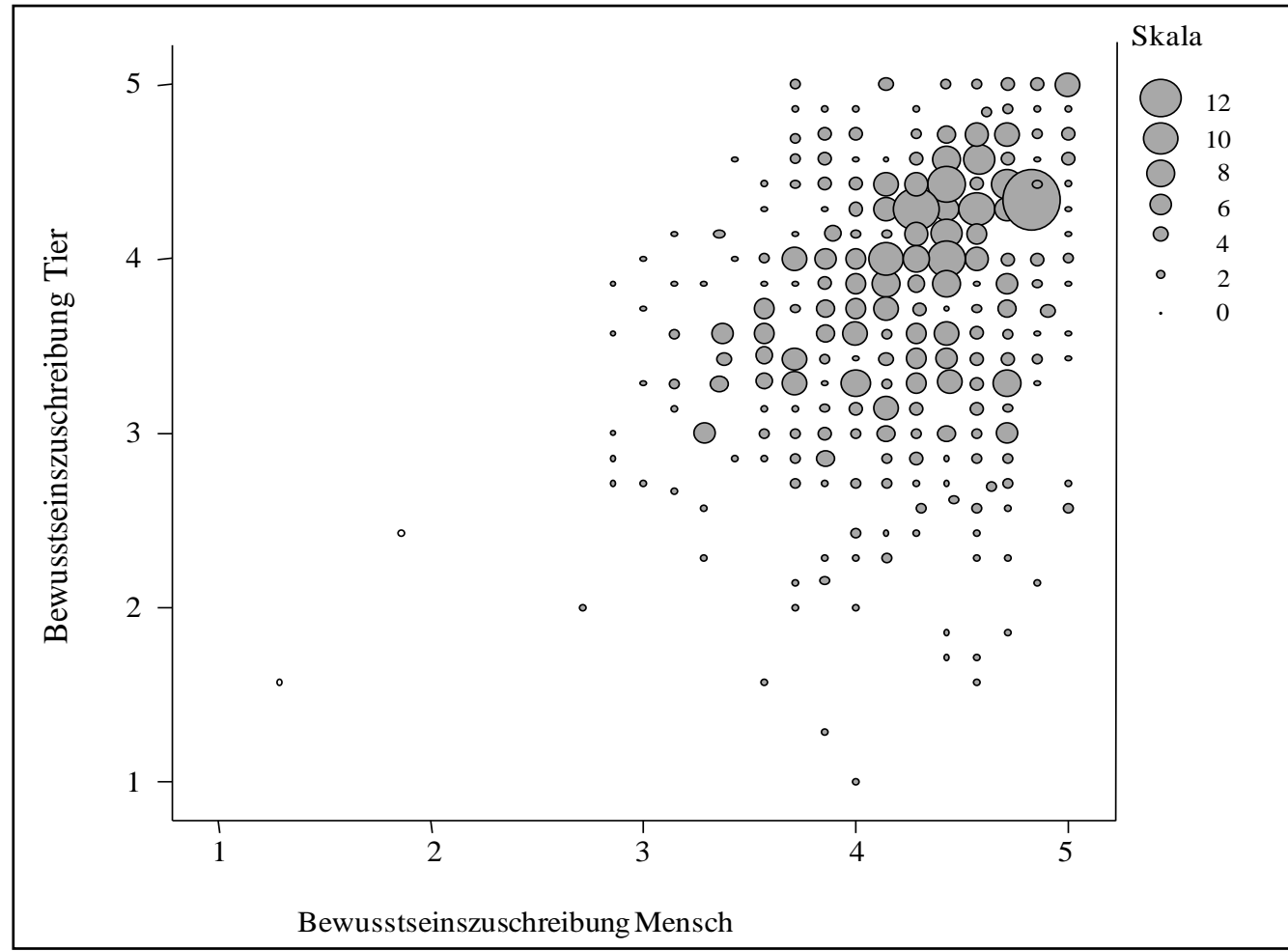

Quelle: Eigene Daten 
Das Streudiagramm zeigt, dass sowohl Menschen als auch Tieren ein hohes Bewusstsein zugeschrieben wird. Befragte, die Menschen ein hohes Bewusstsein zuschreiben haben Tieren in den meisten Fällen ebenfalls ein hohes Bewusstsein unterstellt. Die Punktwolke dehnt sich leicht nach unten aus, was bedeutet, dass einige Teilnehmer Menschen mehr Bewusstsein zuschreiben als Tieren. Einige wenige sind der Meinung, dass weder Tiere noch Menschen ein besonders hohes Bewusstsein haben. Ein T-Test zeigt, dass sich die Mittelwerte der Bewusstseinszuschreibung Mensch ( $\mathrm{MW}=4,02, \mathrm{SD}=0,48)$ auf einem Niveau von $\mathrm{p}<0,01$ hoch signifikant von der Bewusstseinszuschreibung Tier (MW= 3,75, $\mathrm{SD}=0,71$ ) unterscheiden. Mithilfe einer einfaktoriellen ANOVA wurde untersucht, ob es ebenfalls signifikante Unterschiede in der Bewusstseinszuschreibung von Menschen und Tieren zwischen den Fachdisziplinen gibt. Als Voraussetzung für eine einfaktorielle ANOVA müssen die Daten normalverteilt sein, Varianzhomogenität aufweisen, auf unabhängigen Beobachtungen basieren und Intervallskalenniveau haben. Wenn die Stichprobe groß genug und die Gruppengrößen identisch sind, ist die ANOVA stabil gegenüber Verletzungen der Normalverteilung und der Varianzhomogenität (Bühner, Ziegler 2009:373ff). Da die Varianzanalyse jedoch nur Auskunft darüber gibt, ob sich mindestens zwei der untersuchten Mittelwerte signifikant voneinander unterscheiden, wird zudem ein Post-Hoc Test durchgeführt. Dieser gibt konkret Aufschluss darüber, welche Gruppenmittelwerte sich signifikant unterscheiden. Wenn keine Varianzhomogenität angenommen werden konnte, wurde der Post-Hoc Test Tamhane-T2 und bei Varianzhomogenität der Post-Hoc Test Bonferroni durchgeführt. Bei der Bewusstseinszuschreibung von Menschen kann kein signifikanter Unterschied festgestellt werden, bei Tieren besteht jedoch ein signifikanter Unterschied (Sig.) zwischen einigen Fachdisziplinen. Tabelle 4 stellt die signifikanten Ergebnisse des Post-Hoc Tests (Tamhane-T2) dar. 
Tabelle 4: Signifikante Mittelwertsunterschiede sowie Effektgröße nach Cohen für die "Bewusstseinszuschreibung Tier" in den Studienfächern

\begin{tabular}{lllll} 
Studienfach & & \multicolumn{1}{c}{$\begin{array}{c}\text { Mittelwert- } \\
\text { differenz }\end{array}$} & \multicolumn{1}{c}{ Sig. $\begin{array}{c}\text { Effekt } \\
\mathbf{d}_{\text {Cohen }}\end{array}$} \\
\hline \hline \multirow{2}{*}{ Agrarwissenschaften } & BWL/VWL & 0,45 & 0,041 & 0,625 \\
ökologische & Landschaftsökologie & $-0,39$ & 0,016 & 0,658 \\
\cline { 2 - 5 } Agrarwissenschaften & BWL/VWL & 0,72 & 0,000 & 1,104 \\
& Jura & 0,49 & 0,007 & 0,741 \\
\cline { 2 - 6 } BWL/VWL & Psychologie & $-0,46$ & 0,029 & 0,651 \\
& Biologie & $-0,55$ & 0,001 & 0,837 \\
Psychologie & Landschaftsökologie & $-0,85$ & 0,000 & 1,325 \\
\cline { 2 - 6 } Jura & Landschaftsökologie & $-0,39$ & 0,014 & 0,66 \\
\cline { 2 - 6 } & Landschaftsökologie & $-0,61$ & 0,000 & 0,955 \\
\hline
\end{tabular}

Neben der Signifikanz wurde die Effektgröße d nach Cohen berechnet. Diese gibt an, ob die identifizierten Mittelwertsunterschiede als schwacher $(d=0,2)$, mittlerer $(d=0,5)$ oder starker $(d=0,8)$ Effekt zu bewerten sind. Die Effektstärke soll die praktische Bedeutsamkeit der Signifikanz darstellen, da sie anders als die Signifikanz kaum von der Stichprobengröße abhängig ist (Botz, Döring 2006). Demnach stellt die unterschiedliche Bewusstseinszuschreibung für das Tier zwischen den Fachdisziplinen ökologische Agrarwissenschaften und BWL/VWL, Landschaftsökologie und BWL/VWL, Biologie und BWL/VWL sowie zwischen Jura und Landschaftsökologie einen starken Effekt dar. Alle anderen signifikanten Ergebnisse sind als mittlere Effekte zu bewerten.

\subsection{Einstellung zur aktuellen Tierhaltung und Fleischkonsum}

Die Zufriedenheit mit dem aktuellen Zustand der landwirtschaftlichen Nutztierhaltung ist mit einem Mittelwert von 1,83 ( $\mathrm{SD}=0,91, \mathrm{~N}=610)$ relativ gering, wohingegen die Wahrnehmung des Tierleids und -wohls mit einem Mittelwert von 4,45 $(\mathrm{N}=610)$ bei einer Standardabweichung von 0,65 insgesamt sehr hoch ist. Bei einem Mittelwertvergleich in den verschiedenen Fachdisziplinen 
zeigt sich, dass Agrarwissenschaftler, BWL/VWLer und Juristen signifikant zufriedener mit dem Status Quo sind als Landschaftsökologen, ökologische Agrarwissenschaftler und Philosophen. Gleichzeitig sind Agrarwissenschaftlern, BWL/VWLern und Juristen das Wohlbefinden und das wahrgenommene Tierleid signifikant weniger wichtig als Landschaftsökologen, ökologischen Agrarwissenschaftlern und Biologen.

In Tabelle 5 ist die deskriptive Statistik der Variablen Fleischkonsum dargestellt.

\section{Tabelle 5: Fleischkonsum in den Studienfächern}

\begin{tabular}{lcccc} 
& N & Mittelwert & SD & Standardfehler \\
\hline \hline Agrarwissenschaften & 63 & 2,71 & 1,152 & 0,145 \\
ökologische Agrarwissenschaften & 71 & 1,98 & 0,845 & 0,100 \\
BWL/VWL & 52 & 2,90 & 1,283 & 0,178 \\
Psychologie & 65 & 2,26 & 1,253 & 0,155 \\
Philosophie & 42 & 1,67 & 0,992 & 0,153 \\
Jura & 48 & 2,46 & 1,086 & 0,157 \\
Biologie & 63 & 2,01 & 1,032 & 0,130 \\
Landschaftsökologie & 54 & 1,67 & 0,901 & 0,122 \\
\hline \hline
\end{tabular}

Quelle: Eigene Daten

Die Ergebnisse zeigen, dass in den Fachdisziplinen BWL/VWL, Agrarwissenschaften und Jura am meisten Fleisch konsumiert wird, wohingegen in den Fachdisziplinen Landschaftsökologie, Philosophie und ökologische Agrarwissenschaften vergleichsweise wenig Fleisch konsumiert wird.

Anhand einer einfaktoriellen ANOVA wurde untersucht, ob signifikante Unterschiede des Fleischkonsums zwischen den Fachdisziplinen festzustellen sind. 
Tabelle 6: Signifikante Mittelwertsunterschiede sowie Effektgröße nach Cohen für den Fleischkonsum in den Studienfächern

\begin{tabular}{llccc} 
Studienfach & Mittelwert- & Sig. & $\begin{array}{c}\text { Effekt } \\
\text { differenz }\end{array}$ & \\
\hline \hline & Ökologische & \\
Agrarwissenschaften & Philosophie & 0,73 & 0,002 & 0,73 \\
& Biologie & 1,05 & 0,000 & 0,97 \\
& Landschaftsökologie & 0,70 & 0,014 & 0,65 \\
& ökologische & 1,05 & 0,000 & 1,01 \\
\hline \multirow{3}{*}{ BWL/VWL } & Agrarwissenschaften & 0,92 & 0,001 & 0,88 \\
& Philosophie & 1,237 & 0,000 & 1,08 \\
& Biologie & 0,887 & 0,003 & 0,78 \\
Jura & Landschaftsökologie & 1,237 & 0,000 & 1,13 \\
\cline { 2 - 5 } & Philosophie & 0,791 & 0,000 & 0,77 \\
& Landschaftsökologie & 0,791 & 0,004 & 0,81 \\
\hline
\end{tabular}

Quelle: Eigene Daten

Auffällig ist, dass sich die Fachbereiche Agrarwissenschaften und BWL/VWL signifikant von den Fachdisziplinen ökologische Agrarwissenschaften, Philosophie, Biologie und Landschaftsökologie unterscheiden. Bei Jura kann ebenfalls ein signifikanter Unterschied zu Philosophie und Landschaftsökologie identifiziert werden.

Um zu analysieren in welcher Stärke und Richtung die Variablen Status Quo, Tierleid und -wohl sowie Bewusstseinszuschreibung Tier zusammenhängen wurde eine Korrelationsanalyse durchgeführt. Der Korrelationskoeffizient (r) variiert zwischen -1 und 1 . Je höher der errechnete Koeffizient ist, desto stärker ist der Zusammenhang zwischen den analysierten Variablen (Brosius 2013). Auffällig ist die stark negative Korrelation $(r=-0,648, p<0,01)$ zwischen der Zufriedenheit mit dem Status Quo in der landwirtschaftlichen Nutztierhaltung und der Einstellung zum wahrgenommenen Tierleid. Des Weiteren kann eine mittlere negative Korrelation $(\mathrm{r}=-0,449, \mathrm{p}<0,01)$ zwischen der Einstellung zum wahrgenommenen Tierleid und dem Fleischkonsum festgestellt werden. Zudem ergab sich eine mittlere positive 
Korrelation $(\mathrm{r}=0,588, \mathrm{p}<0,01)$ zwischen der Einstellung zum wahrgenommen Tierleid und der Bewusstseinszuschreibung von Tieren.

Eine Regressionsanalyse soll aufdecken, durch welche Variablen die Bewusstseinszuschreibung von Tieren erklärt werden können. Als Prädiktoren werden die Skalen Status Quo sowie Tierleid und -wohl gewählt. Da die beiden Prädiktorvariablen auf einem Niveau von $\mathrm{p}<0,01$ signifikant stark negativ miteinander korrelieren $^{8}(r=-0,648)$ liegt eine Kollinearität vor. Das bedeutet, dass der Koeffizient des einen Prädiktors unterschätzt und der des anderen überschätzt werden kann. Demnach kann die Verteilung des Einflusses der erklärenden Variablen falsch sein. Der geschätzte Gesamteinfluss beider Variablen wird jedoch korrekt geschätzt (Brosius 2013:580). Die Regressionsanalyse ergibt einen Gesamteinfluss beider Variablen auf die Bewusstseinszuschreibung von 34,4 \%.

In einer schrittweisen Regressionsanalyse wurde zudem untersucht, inwiefern der Fleischkonsum durch die Variablen Tierleid und -wohl, die Zufriedenheit mit dem Status Quo und der Bewusstseinszuschreibung von Menschen und Tieren erklärt werden kann.

${ }^{8}$ Zur Orientierung für die Auswertung einer Korrelationsanalyse dienen die Konventionen nach Cohen 


\section{Abbildung 4: Schrittweise Regressionsanalyse zur Erklärung des Fleischkonsums}

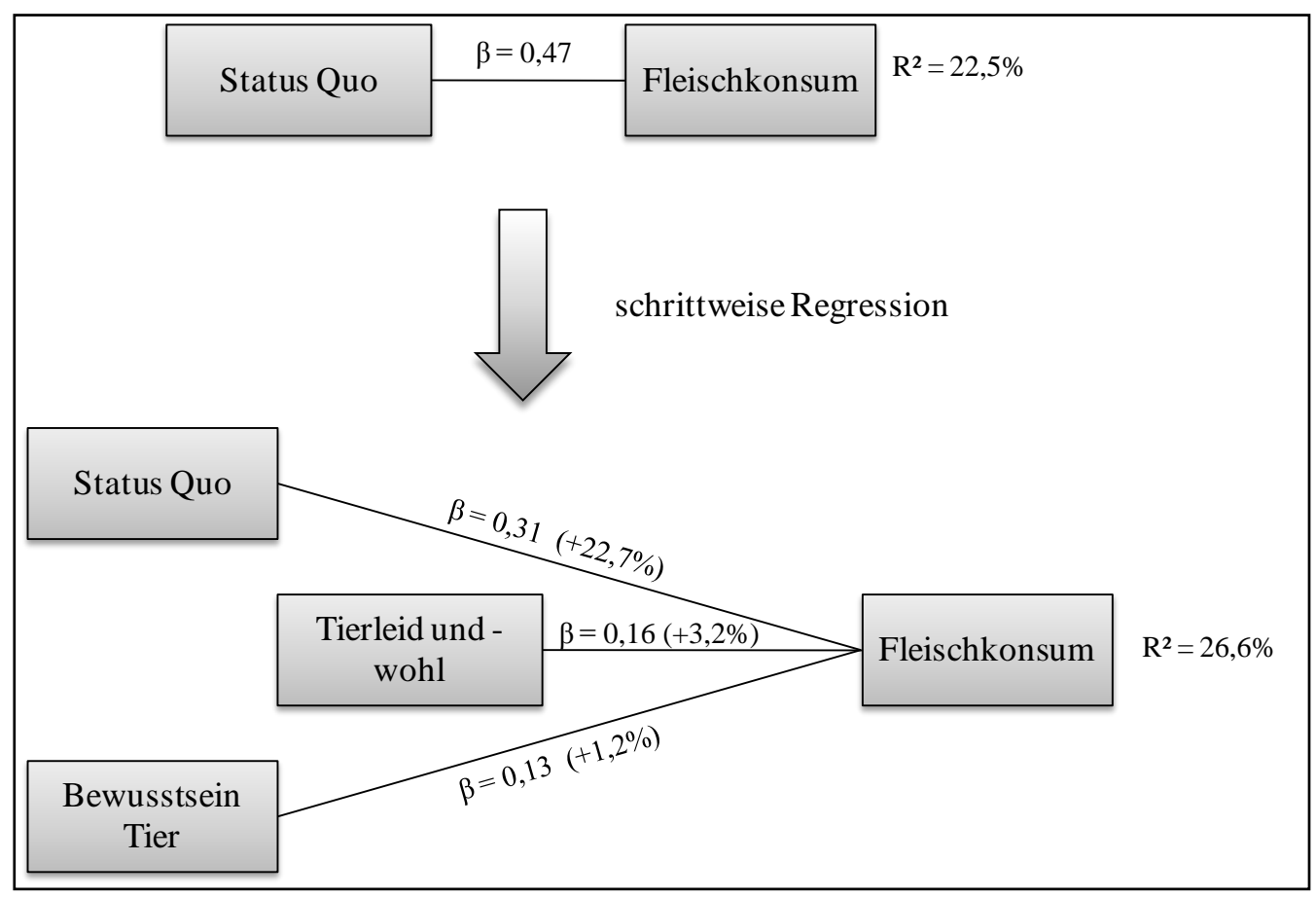

Quelle: Eigene Daten

Abbildung 4 verdeutlicht, dass der angegebene Fleischkonsum zu $\left(\mathrm{R}^{2}=\right.$ Bestimmtheitsmaß) 22,5 \% durch die alleinige Berücksichtigung des Status Quo aufgeklärt wird. Unter dem Einschluss der Variablen Tierleid und -wohl sowie der Bewusstseinszuschreibung Tier können sogar 26,6 \% der Variable Fleischkonsum erklärt werden. Durch die schrittweise Regressionsanalyse wurde die Bewusstseinszuschreibung Mensch ausgeschlossen, da diese keine zusätzliche Aufklärung des Fleischkonsums geliefert hat.

\subsection{Einschätzung und Validität von Präferenz- und Motivationstests}

Nachdem die Teilnehmenden einen Beispieltext zum Präferenz- und Motivationstest gelesen haben, wurden sie zur Einschätzung und zur Validität der Tests befragt. Die Befragten hatten auch bei diesen Fragen die Möglichkeit auf einer endpunktbenannten Intervallskala von 1 „Nein, lehne ab“ bis 5 „Ja, stimme zu“ zu wählen. 
Tabelle 7: Deskriptive Statistik Einschätzung und Validität von Präferenz- und Motivationstests

\begin{tabular}{lcccc} 
& N & Mittelwert & SD & Standardfehler \\
\hline \hline Validität Präferenztest & 455 & 4,2319 & 0,65 & 0,03 \\
Einschätzung Präferenztest & 456 & 4,1535 & 0,79 & 0,03 \\
Validität Motivationstest & 452 & 4,2813 & 0,65 & 0,03 \\
Einschätzung Motivationstest & 453 & 4,0861 & 0,81 & 0,03 \\
\hline \hline
\end{tabular}

Quelle: Eigene Daten

Die Mittelwerte der einzelnen Variablen liegen sehr dicht beieinander. Die Validität des Präferenztests wurde insgesamt mit einem Mittelwert von 4,23 (SD =0,65) bewertet, die Einschätzung von Präferenztests mit 4,15 (SD=0,79), die Validität des Motivationstests mit 4,28 (SD =0,65) und die Einschätzung von Motivationstests mit 4,08 (SD =0,81). Eine Varianzanalyse deckt auf, dass es keine Unterschiede zwischen den Fachdisziplinen und der Einschätzung und Validität von Präferenzund Motivationstests gibt.

\subsection{Zahlungs- und Mehrzahlungsbereitschaft für tierische Produkte aus mit Präferenz- und Motivationstests optimierter Haltung}

Im Rahmen des Fragebogens wurde abgefragt, wieviel die Studierenden für 1 Liter Milch, 6 Eier und $250 \mathrm{~g}$ Hackfleisch bezahlen würden. Des Weiteren wurde abgefragt, ob die Probanden das gefragte Produkt generell kaufen oder nicht. Von den Befragten gaben 18,4\% an keine Milch zu kaufen, 23,3\% keine Eier und 46,5 \% kaufen kein Hackfleisch.

Aus der Befragung ergibt sich eine mittlere Zahlungsbereitschaft von 0,99 € pro Liter Milch, 1,93 € für 6 Eier und 2,70 € für 250 g Hackfleisch. In Abbildung 5 sind die Zahlungsbereitschaften der drei Produkte in Abhängigkeit vom Studienfach dargestellt. 
Abbildung 5: Zahlungsbereitschaft für Milch, Eier und Hackfleisch in Abhängigkeit vom Studienfach

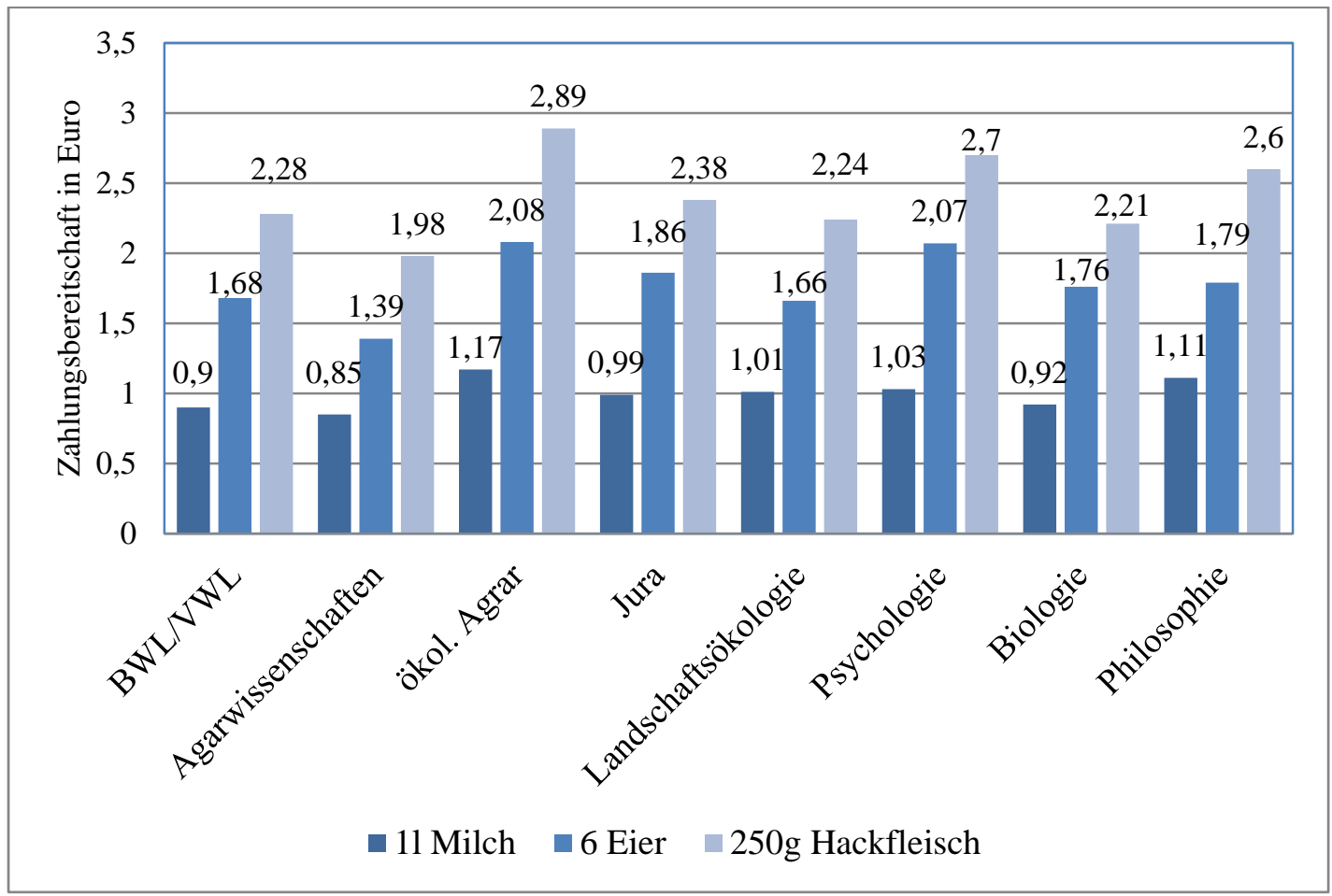

Quelle: Eigene Daten

Durch eine einfaktorielle Varianzanlayse wird untersucht, ob zwischen den Studienfächern ein signifikanter Unterschied in der Zahlungsbereitschaft besteht. In diesem Fall wurde als Post-Hoc Test der Tamhane-T2 durchgeführt, da der LeveneTest auf Varianzhomogenität ergab, dass die Daten keine Varianzhomogenität aufweisen.

Tabelle 8: Signifikante Mittelwertsunterschiede der Zahlungsbereitschaft für 1 l Milch in Abhängigkeit vom Studienfach

\begin{tabular}{llcccc} 
Studienfach & & $\begin{array}{l}\text { Mittlere } \\
\text { Differenz }\end{array}$ & SD & Sig. & $\begin{array}{l}\text { Effekt } \\
\text { d }_{\text {Cohen }}\end{array}$ \\
\hline \hline \multirow{3}{*}{ ökologische } & Agrarwissenschaften & 0,315 & 0,042 & 0,000 & 1,369 \\
Agrarwissenschaften & BWL/VWL & 0,263 & 0,048 & 0,000 & 1,185 \\
& Biologie & 0,247 & 0,046 & 0,000 & 1,028 \\
Philosophie & Agrarwissenschaften & 0,262 & 0,078 & 0,025 & 0,846 \\
\hline \hline
\end{tabular}


In Tabelle 8 ist aufgeführt, zwischen welchen Gruppen signifikante Unterschiede in der Zahlungsbereitschaft für einen Liter Milch bestehen. Ökologische Agrarwissenschaftler haben im Durchschnitt die höchste Zahlungsbereitschaft für einen Liter Milch und unterscheiden sich somit signifikant von Agrarwissenschaftlern, BWL/VWLern und Biologen, wobei letztere durchschnittlich die niedrigsten Zahlungsbereitschaften haben. Zudem unterscheidet sich die Zahlungsbereitschaft von Philosophen und Agrarwissenschaftlern signifikant. Die errechneten Effektstärken (dcohen) ergeben alle einen starken Effekt.

Neben der Zahlungsbereitschaft wurde ebenfalls ermittelt, wieviel die Teilnehmer bereit wären für einen Liter Milch mehr auszugeben, wenn die Kühe, die zur Erzeugung gehalten werden in einem Betrieb stehen, dessen Haltungsbedingungen durch die Ergebnisse aus Präferenz- und Motivationstests verbessert wurden. Abbildung 6 zeigt die Ergebnisse für die einzelnen Studienfächer.

Abbildung 6: Mehrzahlungsbereitschaft für 1 l Milch aus mit Präferenz- und Motivationstests optimierter Haltung in Abhängigkeit vom Studienfach

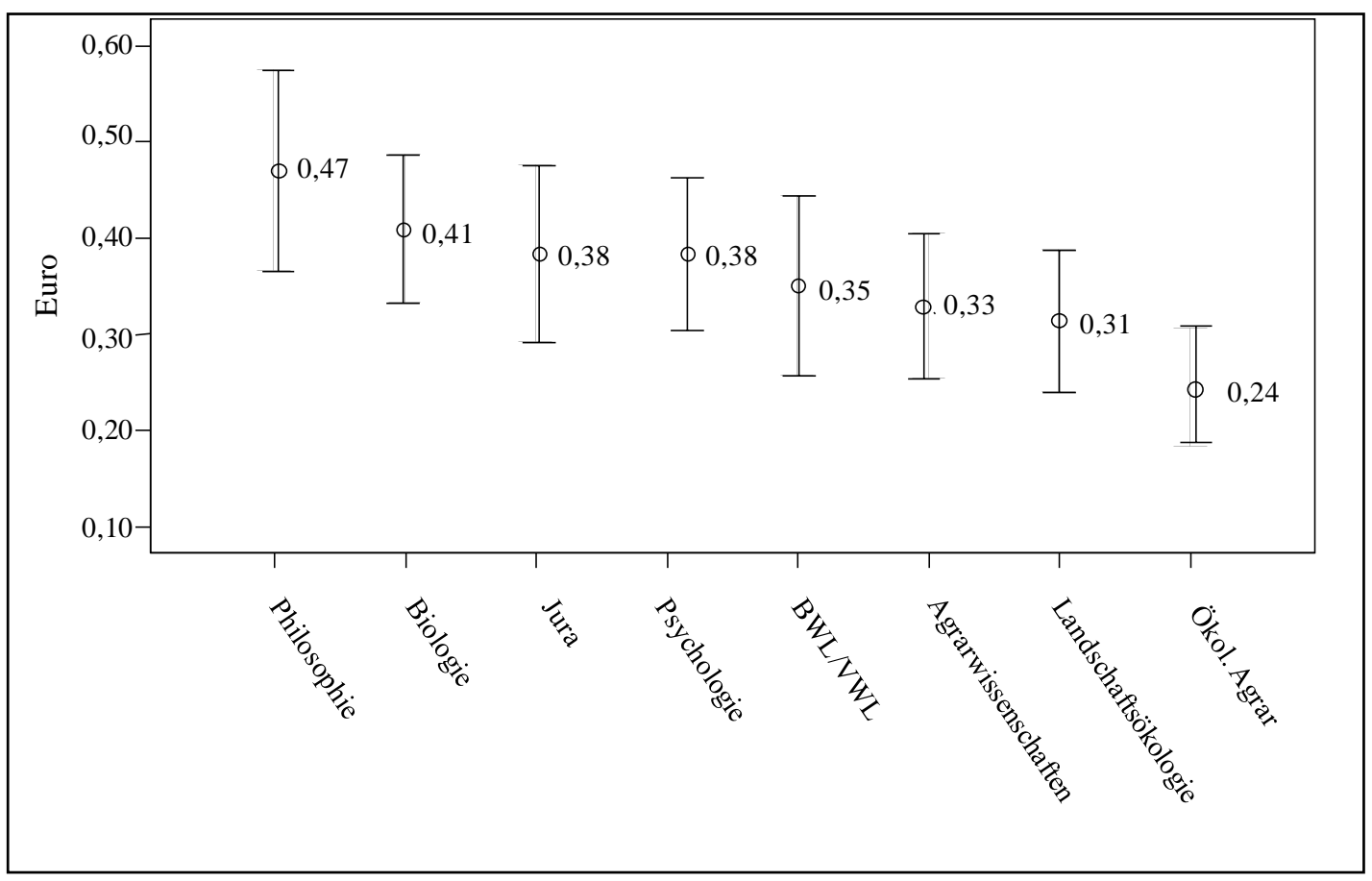

Quelle: Eigene Daten 
Die Mehrzahlungsbereitschaft der Studenten für 1 Liter Milch aus mit Präferenz- und Motivationstests optimierter Haltung beträgt durchschnittlich 35 Cent. Die höchste Mehrzahlungsbereitschaft haben Studenten der Philosophie (47 Cent) und Biologie (41 Cent), die niedrigste Mehrzahlungsbereitschaft mit 24 Cent haben ökologische Agrarwissenschaftler. Die einfaktorielle ANOVA bestätigt, dass es einen signifikanten Unterschied in der Mehrzahlungsbereitschaft der Fachdisziplinen für Milch gibt. Der Post Hoc Test identifiziert für die Mehrzahlungsbereitschaft für Milch einen signifikanten Unterschied zwischen Philosophie und Biologie zu ökologischer Agrarwissenschaft.

Für Eier und Hackfleisch kann kein signifikanter Unterschied in der Mehrzahlungsbereitschaft nachgewiesen werden. Abbildung 7 stellt die Mehrzahlungsbereitschaft für 1 l Milch, 6 Eier und 250 g Hackfleisch dar. Auffällig ist jedoch, dass die Fächer Philosophie, Biologie, Jura und Psychologie in absteigender Reihenfolge bei Milch und Eiern die höchsten Mehrzahlungsbereitschaften aufweisen.

Abbildung 7: Mehrzahlungsbereitschaft für 1 l Milch, 6 Eier und 250 g Hackfleisch aus mit Präferenz- und Motivationstests optimierter Haltung in Abhängigkeit vom Studienfach

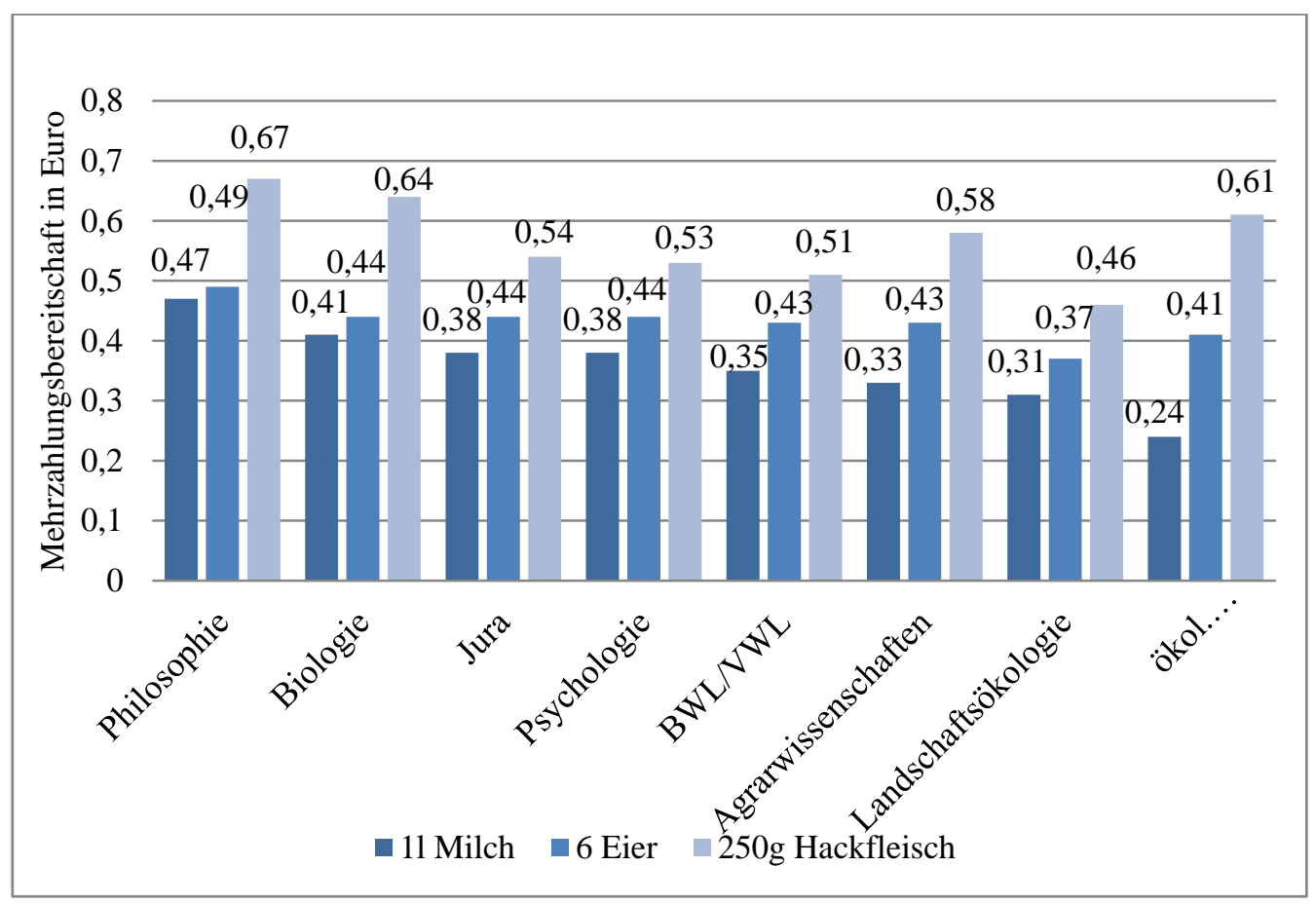

Quelle: Eigene Daten 


\section{Diskussion}

Durch die Studie konnte nachgewiesen werden, dass die Zuschreiung eines Bewusstseins für Tiere (Mittelwert= 3,75) anhand der Charakteristika des Homo oeconomicus im Vergleich zum Menschen (Mittelwert=4,20) signifikant geringer ausfällt. Dieses Ergebnis war zu erwarten, da Menschen zum einen ihre kognitiven Fähigkeiten im Rahmen einer Selbsteinschätzung leichter nachvollziehen und bewerten können. Zum anderen fällt es vielen schwer, sich in Tiere hineinzuversetzen und deren Handlungen und Emotionen einzuschätzen und zu messen. Die Differenz zwischen den Mittelwerten ist allerdings geringer als erwartet, da vor dem Hintergrund der aktuellen Haltungsstandards angenommen wurde, dass die Wahrnehmungsfähigkeit und der Bewusstseinszustand von Tieren deutlich geringer eingeschätzt werden.

Die Beurteilung tierischen Bewusstseins in den verschiedenen untersuchten Fachrichtungen weist signifikante Unterschiede auf. Studierende der Betriebs- und Volkswirtschaftslehre sprechen Tieren signifikant weniger Bewusstsein zu als konventionelle und ökologische Agrarwissenschaftler sowie Biologen. Zudem ist ein signifikanter Unterschied zwischen Jura und Landschaftsökologie festzustellen. Die signifikant geringere Zuschreibung von Bewusstsein von Tieren durch BWL/VWLer und Juristen lässt sich durch den fehlenden Kontakt dieser Fachdisziplinen zu Tieren erklären. Anders als erwartet unterscheidet sich jedoch die Bewusstseinszuschreibung durch BWL/VWLer und Agrarwissenschafter signifikant. Das könnte daran liegen, dass Agrarwissenschaftler Tiere durch den engen Kontakt weniger als reines wirtschaftliches Gut ansehen als Ökonomen. Obwohl der Mittelwert von 3,75 zwar bestätigt, dass es einen wahrgenommenen Unterschied zwischen dem Bewusstsein von Menschen und Tieren gibt, ist dieser deutlich positiv zu bewerten, da das Bewusstsein von Menschen und Tieren nicht grundlegend unterschiedlich bewertet wird.

Die Bewusstseinszuschreibung für Tiere wird zu 34,4 \% durch das wahrgenommene Tierleid und die Zufriedenheit mit den aktuellen Haltungsbedingungen erklärt. Aufgrund der stark negativen Korrelation der Skalen Tierleid und -wohl mit der Skala Status Quo $(\mathrm{r}=-0,648, \mathrm{p}<0,01)$ kann bestätigt werden, dass Probanden, denen das Wohlbefinden des Tieres und die artgerechte Haltung wichtig sind, unzufriedener mit den aktuellen Haltungsbedingungen landwirtschaftlicher Nutztiere 
sind. Aufgegliedert auf die Fachdisziplinen zeigt sich, dass Agrarwissenschaftler, BWL/VWLer und Juristen signifikant zufriedener mit dem Status Quo sind als Landschaftsökologen, ökologische Agrarwissenschaftler und Philosophen. Grundsätzlich ist die Zufriedenheit mit den aktuellen Bedingungen in der landwirtschaftlichen Nutztierhaltung mir einem Mittelwert von 1,83 (SD =0,91) in allen Fachdisziplinen eher gering.

Agrarwissenschaftlern, BWL/VWLern und Juristen sind das Wohlbefinden und das wahrgenommene Tierleid signifikant weniger wichtig als Landschaftsökologen, ökologischen Agrarwissenschaftlern und Biologen. Ökologische Agrarwissenschaftler beschäftigen sich intensiv mit artgerechter Tierhaltung und alternativem Umgang mit Tieren, aus diesem Grund stehen sie den aktuellen Tierhaltungsbedingungen skeptischer gegenüber als Betriebs- und Volkswirte, die zunächst mehr auf den wirtschaftlichen Aspekt der Tierhaltung fokussiert sind.

Bei der Analyse des Fleischkonsums konnte herausgearbeitet werden, dass Studierende aus den Fachbereichen BWL/VWL, Agrarwissenschaften und Jura mehr Fleisch konsumieren als Probanden aus den anderen untersuchten Disziplinen.

Des Weiteren wurde untersucht ob die Faktoren Bewusstseinszuschreibung, Zufriedenheit mit den landwirtschaftlichen Haltungsbedingungen und die Einstellung zum wahrgenommenen Tierleid und dem Wohlbefinden von Nutztieren Einflüsse auf den Fleischkonsum der Befragten haben. Eine schrittweise Regressionsanalyse zeigt, dass die Zufriedenheit mit den aktuellen Haltungsbedingungen in der landwirtschaftlichen Nutztierhaltung $\left(\mathrm{R}^{2}=22,7 \%\right)$ den Haupteffekt auf den Fleischkonsum ausübt. Der Wunsch nach artgerechter Haltung und einem hohen Wohlbefinden für die Tiere mit einem Bestimmtheitsmaß von $\mathrm{R}^{2}=3,2 \%$ sowie die Bewusstseinszuschreibung für Tiere mit $\mathrm{R}^{2}=1,2 \%$ haben dagegen weniger Einfluss auf die Höhe des Fleischkonsums. Das Ergebnis deckt sich mit der Studie des Bundesministeriums für Ernährung, Landwirtschaft und Verbraucher, die 2013 ebenfalls herausfand, dass eine artgerechte Tierhaltung Einfluss auf den Fleischkonsum hat. Der geringe Einfluss der Skala Tierleid und -wohl ist darauf zurückzuführen, dass der Wunsch nach einer artgerechteren Haltung und mehr tierischem Wohlbefinden die Zufriedenheit mit dem Status Quo stark beeinflusst. Eine Erklärungsmöglichkeit ist, dass den Konsumenten von Fleisch andere Aspekte der Nutztierhaltung wichtiger sind als die Artgerechtheit und die Berücksichtigung 
tierischen Wohlbefindens. Eine Forsa Umfrage ergab 2014, dass sich Verbraucher bei Lebensmitteln primär Sorgen um eine Schadstoffbelastung der Lebensmittel machen. Erst an zweiter Stelle folgen Bedenken über eine nicht artgerechte Haltung (Verbraucherzentrale Bundesverband 2014). Demnach könnten Lebensmittelskandale wie beispielsweise Dioxin in Eiern, Pferdefleisch in Lebensmitteln ohne entsprechende Kennzeichnung oder Antibiotika im Fleisch einen höheren Einfluss auf den sinkenden Fleischkonsum haben als Aspekte einer artgerechteren Nutztierhaltung.

Da die Haltungsbedingungen Einfluss auf das Konsumverhalten und auf die Zufriedenheit der Konsumenten mit der landwirtschaftlichen Tierproduktion ausüben, wurde die Akzeptanz bei Verbrauchern untersucht, die Haltungsbedingungen konkret durch die Einbeziehung von Tieren mittels Präferenzoder Motivationstests zu verbessern. Ergebnis der Befragung war, dass die positive Einstellung zu und die eingeschätzte Validität von Präferenz- und Motivationstests in allen Fachbereichen sehr hoch und auch keine Unterschiede zwischen den Fachbereichen feststellbar sind, obwohl erwartet wurde, dass thematisch weiter entfernte Fächer wie Jura oder BWL/VWL den Testverfahren skeptischer gegenüberstehen könnten. Dieses Ergebnis zeigt, dass der Einsatz von Präferenz- und Motivationstests Zustimmung beim Konsumenten findet und auch ihre Ergebnisse anerkannt werden.

Dass die artgerechte Haltung von Tieren für immer mehr Verbraucher eine Rolle spielt wurde bereits durch die Forsa Umfrage der Verbraucherzentrale Bundesverband (2014) und durch die Studie des Bundesministeriums für Landwirtschaft, Ernährung und Verbraucher (2013) bestätigt. Somit deckt sich das Ergebnis dieser Studie mit den bisherigen Forschungsergebnissen. Eine durch die Ergebnisse von Präferenz- und Motivationstests verbesserte Haltung könnte bei Verbrauchern das Bedürfnis nach mehr Artgerechtheit in der Tierhaltung befriedigen, da Tiere direkt in die Entscheidungsfindung zu den optimalen Haltungsbedingungen integriert werden.

Ziel der Befragung war es einerseits herauszufinden, ob Verbraucher aus verschiedenen Fachdisziplinen die Ergebnisse von Präferenz- und Motivationstests als valide einschätzen und andererseits, wie hoch ihre Mehrzahlungsbereitschaft für Produkte ist, die auf Betrieben erzeugt wurden, auf denen die Haltungsbedingungen 
durch die Ergebnisse von Präferenz- und Motivationstests verbessert wurden. In Tabelle 9 sind die Preise für konventionell und ökologisch erzeugte Produkte sowie die von den Studenten angegebene Zahlungs- (ZB) und Mehrzahlungsbereitschaft (MZB) aufgelistet. Der hier angegebene Endpreis für Produkte aus mit Präferenz(PT) und Motivationstests verbesserter Haltung ergibt sich aus der Summe von Zahlungs- und Mehrzahlungsbereitschaft.

Tabelle 9: Marktpreis, Zahlungsbereitschaft und Mehrzahlungsbereitschaft für Milch, Eier und Hackfleisch aus mit Präferenz- und Motivationstests optimierter Haltung

konventionell ökologisch ZB $\quad$ MZB Endpreis

PT/MT

\begin{tabular}{|c|c|c|c|c|c|}
\hline $\begin{array}{l}1 \text { I Milch } \\
\text { (1,5\%, ultrahocherhitzt) }\end{array}$ & $0,70 €$ & $1,09 €$ & $0,99 €$ & $0,35 €$ & $1,34 €$ \\
\hline 6 Eier (Bodenhaltung) & $0,95 €$ & $1,39 €$ & $1,93 €$ & $0,42 €$ & $2,35 €$ \\
\hline $\begin{array}{l}250 \text { g Hackfleisch (Schwein, } \\
\text { Rind) }\end{array}$ & $2,19 €$ & $2,79 €$ & $2,70 €$ & $0,57 €$ & $3,26 €$ \\
\hline
\end{tabular}

Quelle: Eigene Daten

Für 1 Liter Milch wurde eine mittlere Zahlungsbereitschaft von 0,99€ angegeben und eine Mehrzahlungsbereitschaft von durchschnittlich 0,35 € für einen Liter aus mit Präferenz- und Motivationstests optimierter Haltung. Daraus ergibt sich eine prozentuale Mehrzahlungsbereitschaft von 35,35 \% für Milch, 21,76 \% für Eier und 20,71 \% für Hackfleisch. Diese Ergebnisse sind jedoch vorsichtig zu interpretieren, da aufgrund der Thematik höhere Zahlungs- und Mehrzahlungsbereitschaften aus sozialer Erwünschtheit angegeben worden sein könnten.

Die anfangs aufgestellte Hypothese, dass eine höhere Zahlungs- und Mehrzahlungsbereitschaft in den Fachgruppen festgestellt werden kann je unzufriedener die Befragen mit dem aktuellen Stand der landwirtschaftlichen Nutztierhaltung sind und je wichtiger ihnen das Wohlbefinden von Tieren ist kann nicht angenommen werden. Obwohl Landschaftsökologen das Wohlbefinden und die artgerechte Haltung von Nutztieren am wichtigsten sind und sie die höchste Unzufriedenheit mit den aktuellen Haltungsbedingungen in der Nutztierhaltung 
aufweisen, ist ihre Zahlungsbereitschaft für Milch, Eier und Hackfleisch aus mit Präferenz- und Motivationstests verbesserter Nutztierhaltung relativ gering. Ähnlich verhält es sich mit ökologischen Agrarwissenschaftlern, die jedoch eine etwas höhere Zahlungs- und Mehrzahlungsbereitschaft für die Produkte aufweisen.

\section{Fazit}

Die landwirtschaftliche Tierproduktion ist in den vergangen Jahren häufig aufgrund unzureichender Tierhaltungsbedingungen aufgefallen. Rechtliche Rahmenbedingungen liefern Mindeststandards welche sich vermehrt auf die Sicherstellung und Verbesserung der Produktqualität fokussieren. Nur vereinzelt wurden in den letzten Jahren Änderungen zugunsten des tierischen Wohlbefindens in den Tierhaltungsverordnungen durchgesetzt. Ziel der Studie war es einerseits herauszufinden, ob Verbraucher aus verschiedenen Fachdisziplinen die Ergebnisse von Präferenz- und Motivationstests als valide einschätzen und andererseits wie hoch ihre Mehrzahlungsbereitschaft für Produkte ist, die auf Betrieben erzeugt wurden, auf denen die Haltungsbedingungen durch die Ergebnisse von Präferenz- und Motivationstests verbessert wurden.

Unter einem Präferenztest wird die Möglichkeit verstanden, durch gezielte Versuchsanordnungen Handlungspräferenzen von Tieren zu ermitteln. Um herauszufinden, ob ein Tier beispielsweise eine bestimmte Präferenz für eine Einrichtungsalternative zeigt, werden mehrere Räume mit verschiedenen Einrichtungsgegenständen präpariert. Je nach dem wie häufig das Tier den Einrichtungsgegenstand aufsucht oder wieviel oder wenig Zeit es bei diesem verbringt, desto wichtiger oder unwichtiger ist ihm dieser. Der Motivationstest ist ähnlich strukturiert wie der Präferenztest. Neben der Präferenz soll jedoch zusätzlich erfasst werden, wie hoch die Motivation des Tieres ist, an einen bestimmten Einrichtungsgegenstand zu gelangen. Dazu werden Türe vor die Räume montiert, welche sukzessive mit Gewichten beschwert werden. Durch die Bereitwilligkeit des Tieres dieses Hindernis zu überwinden und dabei Körperenergie einzusetzen kann ermittelt werden, welche „Zahlungsbereitschaft“ das Tier für die jeweiligen Einrichtungsoptionen zeigt. Ist das Tier mit allen Optionen vertraut, kann die Anzahl 
oder Länge der Besuche in den unterschiedlichen Räumen für die Interpretation der Präferenz genutzt werden (Boe et al. 2011; Abou-Ismail, Mahboub 2010).

Die vorliegende Studie basiert auf einer Umfrage unter Studierenden $(\mathrm{N}=619)$ verschiedener Fachrichtungen mit dem Fokus auf ökologischen und konventionellen Agrarwissenschaften, Betriebs- und Volkswirtschaftslehre, Jura, Philosophie Psychologie, Biologie sowie Landschaftsökologie. Durch die Fokussierung auf ein hohes Bildungsniveau der Teilnehmer kann sichergestellt werden, dass diese über Grundkenntnisse der Lebensmittelproduktion, Tierhaltung und Produktqualität verfügen.

Die Ergebnisse der Studie zeigen, dass die Befragten unzufrieden mit dem aktuellen Zustand der landwirtschaftlichen Nutztierhaltung sind und sich artgerechtere Haltungsbedingungen für Nutztiere wünschen. Der Wunsch nach einer artgerechteren Haltung hat jedoch keinen primären Einfluss auf die Höhe des Fleischkonsums. Es werden vielmehr Verbraucherunsicherheiten durch Skandale und Schadstoffbelastungen der Lebensmittel als Ursache für den Verzicht auf Fleisch vermutet.

Innerhalb der berücksichtigten Studienfächer ist eine hohe Mehrzahlungsbereitschaft für Produkte festzustellen, die von Betrieben stammen, deren Haltungsbedingungen durch Präferenz- und Motivationstests verbessert wurden. Für einen Liter Milch konnte eine Mehrzahlungsbereitschaft von 35,35 \%, für Eier eine MZB von 21,76 \% und eine MZB von 20,71 \% für Hackfleisch gegenüber den aktuellen Marktpreisen ermittelt werden. Dies kann einerseits als Hinweis auf das hohe Bewusstsein für die Missstände in der konventionellen landwirtschaftlichen Nutztierhaltung angesehen werden und andererseits als Bereitschaft die Verbesserungen in der Nutztierhaltung durch höhere Verbraucherpreise zu unterstützen und voranzutreiben.

Der Wunsch nach einer artgerechteren Tierhaltung und die Bereitschaft für bessere Haltungsbedingungen höhere Verbraucherpreise in Kauf zu nehmen, kann als eine Distanzierung der Studierenden von den aktuellen Umständen der landwirtschaftlichen Produktion verstanden werden. Dies könnte den Akteuren der Landwirtschaft als Impuls dienen, die Umstände der Produktion tierischer Erzeugnisse an die ethischen und moralischen Ansprüche der Konsumenten schrittweise anzunähern. 
In der durchgeführten Studie konnte eine hohe Akzeptanz für Präferenz- und Motivationstests nachgewiesen werden. Demnach könnten diese Testverfahren dazu eingesetzt werden, die Haltungsbedingungen von landwirtschaftlichen Nutztieren nachhaltig zu verbessern. 


\section{Literaturverzeichnis}

Abou-Imail, U., Mahboub, H. 2011: The effects of enriching laboratory cages using varios physical structures on multiple measures of welfare in singly-housed rats, Laboratory Animals, Vol. 45, S. 145-153

Baur, N., Fromm, S. 2008: Datenanalyse mit SPSS für Fortgeschrittene, Ein Arbeitsbuch, 2. Auflage, Verlag für Sozialwissenschaften, Wiesbaden

Benson, J., Rollin, B. 2004: The well-being of farm animals: challenges and solutions, Blackwell Publishing

Bessei, W., Tinz, J., Reiter, K. 2001: Die Präferenz von Mastkaninchen für Kunststoffgitter und Tiefstreu bei unterschiedlichen Temperaturen, 12. Arbeitstagung über Haltung und Krankheiten der Kaninchen, Pelztiere und Heimtiere, Deutsche Veterinärmedizinische Gesellschaft, S. 133-140

Boe, K., Ehrlenbruch, R., Andresen, I. 2011: The preference for water nipples vs. bowls in dairy goats, Acta Veterinaria Scandinavica, Vol. 53, S. 50-55

Bortz, J., Döring, N. 2006: Forschungsmethoden und Evaluation für Human- und Sozialwissenschaftler. 4. Auflage, Springer, Berlin, Heidelberg

Bräuer, I., Suhr, A. 2005: Ergebnisse von Zahlungsbereitschaftsanalysen Interpretation und Verwendung, in: Marggraf, R., Bräuer, I., Fischer, A., Menzel, S., Stratmann, U., Suhr, A.: Ökonomische Bewertung bei umweltrelevanten Entscheidungen, Einsatzmöglichkeiten von Zahlungsbereitschaftsanalysen in Politik und Verwaltung, Metropolis Verlag, Marburg, S. 149-183

Broom, D. 1991: Animal welfare: Concepts and measurement, Journal of Animal Science, Vol. 69, S .4167-4175

Broom, D. 1993: A usable definition of animal welfare, Journal of Agricultural and Environmental Ethics, Vol. 6, S. 15-25

Brosius, F. 2013: SPSS 21, mitp Verlag, Heidelberg, München

Bubier, N. 1996: The behavioural priorities of laying hens: the effect of cost/ no cost multi-choice tests on time budget, Behavioral Processes, Vol. 37, S. 225-238 
Bühner, M., Zigeler, M. 2009: Statistik für Psychologen und Sozialwissenschaftler, Pearson, München

Bund ökologische Lebensmittelwirtschaft (BÖLW) 2012: Zahlen, Daten, Fakten-Die Bio- Branche 2012, URL: http://www.boelw.de/uploads/pics/ZDF/ZDF_Endversion_120110.pdf, Abrufdatum: 03.12.2014

Bundesministerium für Ernährung, Landwirtschaft und Verbraucherschutz (BMELV) 2012: Mehr Tierschutz in der Legehennenhaltung, URL: http://www.bmelv.de/SharedDocs/Standardartikel/Landwirtschaft/Tier/Tierhalt ung/HaltungLegehennen.html, Abrufdatum 10.12.2012

Bundesministerium für Landwirtschaft, Ernährung und Verbraucherschutz 2013: Ökobarometer 2013 Repräsentative Bevölkerungsbefragung, URL: http://www.bmel.de/SharedDocs/Downloads/Ernaehrung/Oekobarometer_2013 .pdf?_blob=publicationFile, Abrufdatum:03.12.2014

Carpenter, E. 1980: Animals and Ethics- A report of the working party convened by Edward Carpenter, Watkins \& Dulverton, London

Dawkins, M. 1982: Leiden und Wohlbefinden bei Tieren: ein Beitrag zu Fragen der Tierhaltung und des Tierschutzes, Ulmer Fachbuch, Hohenheim

Dawkins, M. 1983: Battery hens name their price: Consumer Demand Theory and the measurement of ethological needs, Animal Behavior, Vol. 31, S. 11951205

Dawkins, M. 1988: Animal Suffering. The science of animal welfare, Chapman Hall, London, New York

Dawkins, M. 1997: D.G.M. Wood-Gush memorial lecture: Why has there not been more progress in animal welfare research?, Applied Animal Behavior Science, Vol. 53, S. 59-73

Deutscher Tierschutzbund 2013: Pelztierfarmen in Deutschland, URL: http://www.tierschutzbund.de/pelztierfarmen.html, Abrufdatum: 30.01.2013

Doosje, B., Ellemers, N., Spears, R. 1995: Perceived intragroup variability as a function of group status and identification, Journal of Experimental Social Psychology, Vol. 31, S. 410-436 
Duncan, I. 1993: Welfare is to do with what animals feel, Journal of Agricultural and Environmental Ethics, Vol. 6, S. 8-14

Duncan, I. 2005: Science-based assessment of animal welfare: farm animals, Revue scientifique et technique International Office of Epizootics, Vol. 24, Issue 2, S. 483-492

Fraser, D., Weary, D., Pajor, E., Milligan, B. 1997: A scientific conception of animal welfare that reflects ethical concerns, Animal Welfare, Vol. 6, S. 187-205

Gonyou, H. 1994: Why the study of animal behavior is associated with the animal welfare issue, Journal of Animal Science, Vol. 72, S. 2171-2177

Hassenstein, B. 1993: Zur Erkennbarkeit von Leiden bei Tieren, in: Leiden und Verhaltensstörungen bei Tieren, Tierhaltung Band 23, Birkhäuser Verlag Berlin, S. 85-92

Heertje, A., Wenzel, H. 2002: Grundlagen der Volkswirtschaftslehre, 6. Auflage, Springer, Berlin

Koistinen, T., Orjala, H., Mononen, J., Korhonen, H. 2008: Position of operant cost affects blue foxes' time budget between sand floor and mesh floor. Applied Animal Behavior Science, Vol. 116, S. 266-272

Manser, C., Broom, D., Overend, P., Morris, T. 1998: Operant studies to determine the strength of preference in laboratory rats for nest-boxes and nesting materials, Laboratory Animals, Vol. 32, S. 36-41

Marggraf, R. 2005: Ökonomische Grundlagen der Umweltbewertung, in: Marggraf, R., Bräuer, I., Fischer, A., Menzel, S., Stratmann, U., Suhr, A.: Ökonomische Bewertung bei umweltrelevanten Entscheidungen, Einsatzmöglichkeiten von Zahlungsbereitschaftsanalysen in Politik und Verwaltung, Metropolis Verlag, Marburg

Martin, G. 1996: Zur naturwissenschaftlichen Erfassbarkeit von Leiden bei Tieren eine Einführung, Tierärztliche Umschau, Vol. 51, S. 131-136

Mason, G. J., Coopert, J., C. Clarebrough 2001: Frustrations of fur-farmed mink, Nature, Art. 410, S. 35-36 
Mason, J., Wang, S., Yehuda, R., Riney, S., Charney, D., Southwick, S. 2001: Psychogenic lowering of urinary cortisol levels linked to increased emotional numbing and a shame-depressive syndrome in combat-related posttraumatic stress disorder, Psychosom Med, Vol. 63, S. 387-401

May, H. 2008: Handbuch zur ökonomischen Bildung, 9. Auflage, Oldenbourg Verlag McGlone, J. 1993: What is animal welfare?, Journal of Agricultural an Environmental Ethics, Vol. 6, S. 26-36

Meixner, O., Pöchtrager, S., Schwarzbauer, A. 2012: Coporate Social Responsibility in der Agrar- und Ernährungswirtschaft, Springer, Berlin

Miele, M., Veissier, I., Evans, A., Botreau, R. 2011: Animal welfare: Establishing a dialogue between science and society, Animal Welfare, Vol. 20, S. 103- 117

Paschke, D. 2004: Grundlagen der Volkswirtschaftslehre, 4. Auflage, PD-Verlag, Heidenau

Renema, R., Robinson, F. 2004: Defining normal: Comparison of feed, restriction and full feeding of female broiler breeders, World's Poultry Science Journal, Vol. 60, Issue 4, S. 508-522

Sambraus, H. 1991: Tierschutz, Naturwissenschaft und Ethologie, Archiv Tierzucht Dummerstorf, Vol. 35, S. 181-192

Sandøe, P. 1996: Animal and Human Welfare- are they the same kind of thing? Acta Agriculturae Scandinavica, Section A (Animal Science), Supplement 27, S. 1115

Schilling, J., Zeller, S. 2007: Soziale Arbeit, Geschichte, Theorie, Profession, 3. Auflage, UTB Verlag

Seaman, S., Waran, N., Mason, G., D‘Eath, R. 2008: Animal economics: assessing the motivation on female laboratory rabbits to reach a platform, social contact and food, Animal Behaviour, Vol. 75, S. 31-41

Sherwin, C., Nicol, C. J. 1996: Reorganisation of behaviour in laboratory mice, mus musculus, with varying cost of access to resources, Animal Behavior, Vol. 51, S. 1087-1093

Tierschutzgesetz (TSchG) in der Fassung der Bekanntmachung vom 18. Mai 2006 
Tierschutznutztierverordnung 2006: Verordnung zum Schutz landwirtschaftlicher Nutztiere und anderer zur Erzeugung tierischer Produkte gehaltener Tiere bei ihrer Haltung URL: http://www.gesetze-im-internet.de/tierschnutztv/, Abrufdatum 17.01.2013

Tullock, G. 1971: The coal tit as a careful shopper, The American Naturalist, Vol. 105, Issue: 941 , S. $77-80$

Van Putten, G. 1992: Forschungsergebnisse und Erkenntnisse zur tiergerechten Schweinehaltung, Züchtungskunde 64 (3/4), S. 209-216

Verbraucherzentrale Bundesverband 2014: Lebensmittel und ihre Umweltauswirkungen, URL: 2014http://www.vzbv.de/cps/rde/xbcr/vzbv/ Lebensmittel-Umwelt-Umfrage-kurzfassung-vzbv-forsa-2014.pdf, Abrufdatum: 08.10.2014

Weber, R., Zárate, A. 2005: Der Begriff Wohlbefinden in der NutztierhaltungDiskussion aktueller Definitionsansätze als Grundlage für praxisorientierte Forschung am Beispiel Mastschweinehaltung, Archiv Tierzucht Dummerstorf, Vol. 48, S. 475-489

Webster, J. 2005: Animal Welfare: Limping towards Eden, UFAW Animal Welfare Series, Blackwell Publishing

Welmesfelder, F. 2007: How animals communicate quality of life: the qualitative assessment of behavior, Animal Welfare, Vol. 16, S. 25-31

Winterhalder, K 1994: Bald neue Haltungsverordnung, Landwirtschaftliches Wochenblatt, Organ des Landesbauernverbandes in Baden Württemberg, Vol. 161, Issue 6, S. 30-31

Wittmann, K., Schoberger, R. 2010: Der Mensch in Umwelt, Familie und Gesellschaft, 8. Auflage, Facultas Verlag 


\section{Fachdisziplinen im Wertekreis}

\section{-Einstellungen zur landwirtschaftlichen Nutztierhaltung}

\section{Inhaltsverzeichnis}

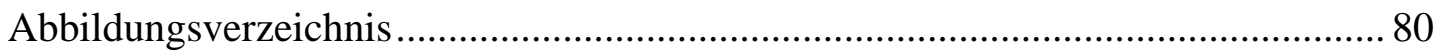

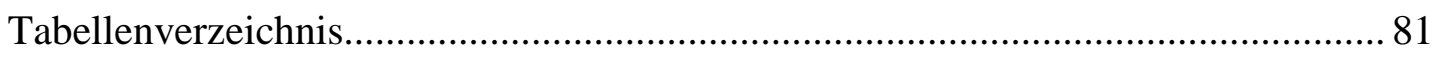

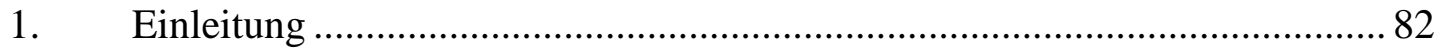

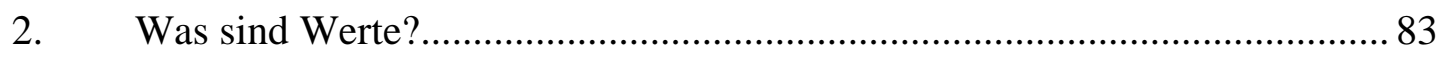

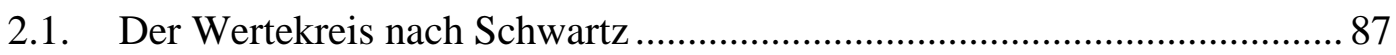

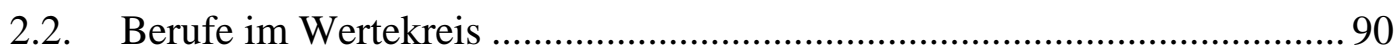

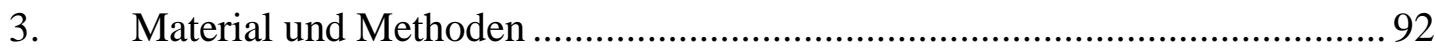

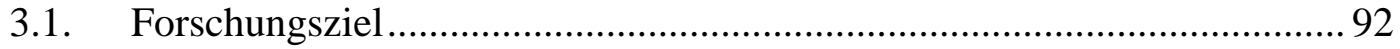

3.2. Fragebogenkonstruktion und Datenerhebung.......................................... 92

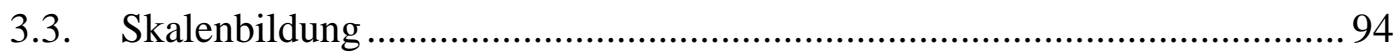

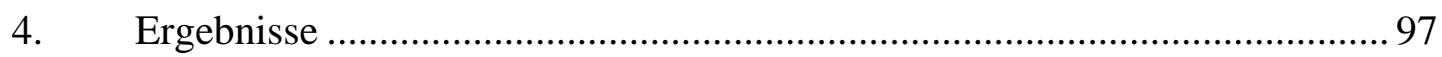

4.1. Beschreibung der Stichprobe................................................................ 97

4.2. Einstellungen zur landwirtschaftlichen Nutztierhaltung in verschiedenen

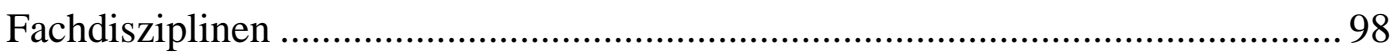

4.3. Studenten unterschiedlicher Fachbereiche im Wertekreis ........................ 101

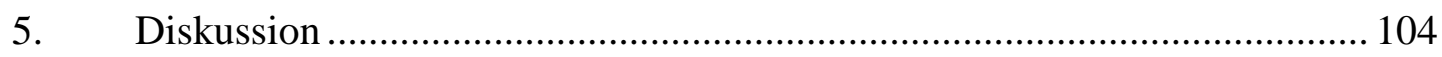

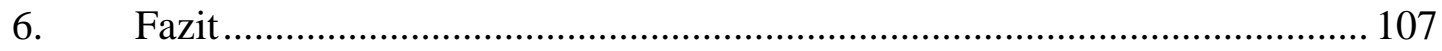

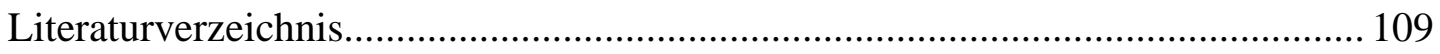




\section{Abbildungsverzeichnis}

Abbildung 1: Der Wertekreis nach Schwartz 88

Abbildung 2: Berufsgruppen im Wertekreis . 91

Abbildung 3: Häufigkeit der Studienfächer. 97

Abbildung 4: Positionierung der Stichprobe im Wertekreis 101

Abbildung 5: Studienfächer im Wertekreis 102

Abbildung 6: Identifikation mit dem Studienfach konventioneller und ökologischer Agrarwissenschaftler im Wertekreis 103 


\section{Tabellenverzeichnis}

Tabelle 1: Die zehn Wertetypen nach Schwartz ..................................................... 86

Tabelle 2: Reliabilitätsanalyse für die Skalenbildung.............................................. 95

Tabelle 3: Signifikante Mittelwertsunterschiede sowie Effektgröße nach Cohen für die "Bewusstseinszuschreibung Tier" in den Studienfächern.................................... 98

Tabelle 4: Fleischkonsum in den Studienfächern ..................................................... 99

Tabelle 5: Signifikante Mittelwertsunterschiede sowie Effektgröße nach Cohen für

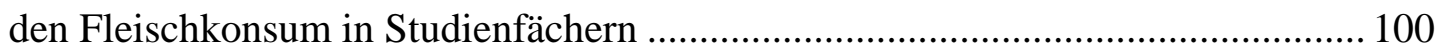




\section{Einleitung}

Nahrungsmittel dienen primär der Ernährung und damit der Aufrechterhaltung von Leben. Sie sind jedoch ebenfalls eng mit kultureller Identität und gesellschaftlicher Zugehörigkeit verbunden und können als Indikatoren für den sozialen Status herangezogen werden. Der technische Fortschritt, die Umstrukturierung der Agrarpolitik und die Globalisierung der Märkte haben einen Wandel in der grundsätzlichen Orientierung, in Lebensstilen und Wertehaltungen hervorgerufen (Wiesinger 2005).

1960 wurden in Deutschland noch 38 \% der Konsumausgaben für Nahrungsmittel, Getränke und Tabakwaren verwendet, 2013 waren es nur noch 15,2 \% (Statistisches Bundesamt 2014) und das obwohl Lebensmittel aufgrund von zunehmender Konkurrenz um Anbauflächen und steigenden Produktions- und Transportkosten insgesamt immer teurer werden. Das ist auf einen im Vergleich zu den Lebensmittelpreisen stärkeren Anstieg der Einkommen zurückzuführen. Beim Erzeuger kommt jedoch immer weniger an. 2009 betrug der Anteil der landwirtschaftlichen Verkaufserlöse an den Verbraucherausgaben für Nahrungsmittel $21 \%$. Anfang der 70er Jahre war der Anteil mit 47,5 \% dagegen noch fast doppelt so hoch (Deutscher Bauernverband 2011).

Der sinkende Erlös führt dazu, dass in der landwirtschaftlichen Produktion immer weniger Ressourcen für Umstrukturierungen und Verbesserungen in der Tierhaltung zur Verfügung stehen. Langfristig muss aus diesem Grund die Ernährung und damit das Bewusstsein für qualitativ hochwertige Lebensmittel und ihre Preise wieder an Bedeutung gewinnen. Dieses Ziel kann nur dann erreicht werden, wenn es zu einer Neuorientierung der Werte auf Seiten der Konsumenten und Produzenten in der Landwirtschaft kommt.

„Es ist nicht schwierig Entscheidungen zu treffen, wenn man seine Werte kennt“ Roy Disney ${ }^{9}$

\footnotetext{
${ }^{9}$ u.a. zitiert in Covey, Merrill 2009:84
} 


\section{Was sind Werte?}

In der heutigen Gesellschaft wird durch Richtlinien, Gesetze und Verordnungen vorgegeben, in welchem Rahmen sich Handlungen und Verhaltensweisen bewegen sollten. Diese Normen werden für alle Lebensbereiche festgelegt. Normwerte existieren nicht nur, um technische oder mechanische Standards zu definieren, sondern auch um gesellschaftliche oder soziale Normen festzulegen. Normen sind konkrete verhaltensorientierte Regeln, in denen situationsabhängig erwünschtes Verhalten determiniert wird. Sie liefern einen eindeutigen Rahmen für gesellschaftlich akzeptiertes bzw. abgelehntes Verhalten und daraus resultierende Konsequenzen. Es existieren jedoch keine weltweit einheitlichen Normen, sie unterschieden sich zum Teil in den unterschiedlichen Gesellschaften und Kulturkreisen enorm und lassen sich darauf zurückführen, was in der jeweiligen Gesellschaft von der Mehrheit als erstrebenswert und richtig eingestuft wird (Scherr 2013). Demnach entwickeln sich Normen aus einer Zusammenfassung von Werten. In Anlehnung an Kluckhohn (1951) und Rokeach (1973) definiert Schwartz den Begriff Werte wie folgt:

“...human values are defined as desirable goals, varying in importance, that serve as guiding principles in people‘s lives. “ (Schwartz 1994:88).

Werte sind nicht nur dazu geeignet um kulturelle Gruppen, Gesellschaften oder Individuen zu charakterisieren, sondern auch um die Motivation von Einstellungen und Verhalten zu erklären (Schwartz 2012:3). Jedes Individuum hat eine Vielzahl an Werten mit einer unterschiedlichen persönlichen Gewichtung. Die individuellen Werte einer Person sind in jeder Lebenssituation allgegenwärtig und beschreiben was dieser wichtig ist. Ist einem Individuum beispielsweise „Leistung“ besonders wichtig, so ist dieser Wert in allen Lebensbereichen wie Schule, Beruf und Freizeit präsent (Bardi, Schwartz 2003:1208). Folgende Aussagen werden von Schwartz über Werte getroffen: 
- $\quad$ Werte sind Überzeugungen, die Handlungen untrennbar beeinflussen.

- Werte beziehen sich auf wünschenswerte Ziele und motivieren Handlungen.

- Werte transzendieren Handlungen und Situationen, sie sind nicht nur in bestimmten Situationen relevant sondern allgegenwärtig.

- $\quad$ Werte dienen als Standards oder Kriterien. Sie leiten Entscheidungen oder Einschätzungen von Handlungen der Politik, von Personen und Events.

- $\quad$ Werte werden individuell nach ihrer Bedeutung zueinander geordnet.

- Werte beeinflussen das Handeln wenn sie im Kontext für den Handelnden relevant sind. Die Bedeutung von Werten ist situationsabhängig (Schwartz 2012:4).

Schwartz ist der Meinung, dass die Ausprägungen der Werte eines Individuums auf die Bedeutung der Werte innerhalb der kulturellen Gesellschaft zurückzuführen sind. Normen, Verfahren und Institutionen helfen den Menschen das für die Gesellschaft angemessene Verhalten $\mathrm{zu}$ zeigen und so entsprechende Werteprioritäten $\mathrm{zu}$ entwickeln (Schwartz 1994:92). Wenn in einer Gesellschaft beispielsweise beruflicher Erfolg, Reichtum und Leistung hoch angesehen sind, so werden die Mitglieder dieser Gesellschaft versuchen diese Ideale zu erfüllen. Hält sich eine gesellschaftliche Gruppe an die gleichen Normen, Verfahren und Institutionen, so werden ähnliche Werteprioritäten ausgebildet.

Dies muss jedoch nicht unbedingt auf eine räumlich zusammenhängende Gesellschaft beschränkt werden, denn beispielsweise arbeiten, handeln und interagieren Individuen unterschiedlicher Berufsgruppen ebenfalls nach spezifischen Maximen. Durch die Inhalte und Werte der verschiedenen Schwerpunkte werden erneut Normen und Verfahren aufgestellt, an denen sich das Individuum orientieren muss. Nach Schwartz müssten auch die hier aufgestellten Ideale das Verhalten einer Fächergesellschaft beeinflussen. Dabei ist an dieser Stelle nicht relevant, ob sich Individuen mit bestimmten Werten für eine Fachrichtung entscheiden oder ob die Lehrinhalte die Gewichtung der Werte von den Studierenden beeinflussen (Bardi, Schwartz 2003:1209). Die Werte eines Individuums bleiben während seiner Entwicklung vom Kind bis hin zum Erwachsenen sehr stabil und verändern sich bis 
ins hohe Alter nicht mehr grundlegend (Bardi, Schwartz 2003:1208). Einzig die Gewichtung der einzelnen Werte kann sich verändern (Knafo, Sagiv 2004:256).

Individuen fühlen sich immer denjenigen Gruppen und Organisationen zugehörig, die kompatibel mit dem eigenen Charakter sind und es erlauben die individuelle Persönlichkeit zu entfalten sowie Ziele zu erreichen (Schneider 1987). Nach diesem Muster wählen Studenten auch ihre Hauptfächer entsprechend ihren Interessen, Fähigkeiten und Motivationen aus. Knafo und Sagiv postulieren, dass die individuellen Werte durch eine Umstrukturierung der Hierarchie an das Arbeitsumfeld angepasst werden können, damit diese besser zu den hier gültigen Werten passen. Individuen innerhalb eines Fachgebietes entwickeln sich aus diesem Grund hinsichtlich ihrer Werte sehr ähnlich (Knafo, Sagiv 2004:256).

Nach Schwartz können Werte zu zehn motivational unterschiedlichen Wertetypen zusammengefasst werden, die aus drei universellen Bedürfnissen menschlicher Existenz (biologische Bedürfnisse, Notwendigkeit koordinierter sozialer Interaktion, Bedürfnis nach Funktionsfähigkeit und Überleben von Gruppen) abgeleitet werden können (Schwartz 1992). 
Tabelle 1: Die zehn Wertetypen nach Schwartz

\begin{tabular}{|c|c|c|}
\hline Wertetyp & Definition & Eingeschlossene Werte \\
\hline Universalismus & $\begin{array}{l}\text { Verständnis, } \\
\text { Wertschätzung, Toleranz } \\
\text { und Schutz für das } \\
\text { Wohlbefinden aller } \\
\text { Menschen und der Natur }\end{array}$ & $\begin{array}{l}\text { Gleichheit, Einheit mit der } \\
\text { Natur, Weisheit, soziale } \\
\text { Gerechtigkeit, Weltoffenheit, } \\
\text { die Umwelt schützen, eine Welt } \\
\text { in Frieden }\end{array}$ \\
\hline Selbstbestimmung & $\begin{array}{l}\text { Unabhängiges Denken und } \\
\text { Handeln, erforschen }\end{array}$ & $\begin{array}{l}\text { Freiheit, Neugierde, Kreativität, } \\
\text { Unabhängigkeit, eigene Ziele } \\
\text { wählen, Selbstrespekt }\end{array}$ \\
\hline Stimulation & $\begin{array}{l}\text { Abwechslung, Neuheiten, } \\
\text { Herausforderungen }\end{array}$ & $\begin{array}{l}\text { Aufregendes Leben, Wagemut, } \\
\text { Abwechslung, sich trauen }\end{array}$ \\
\hline Hedonismus & $\begin{array}{l}\text { Vergnügen und sinnliche } \\
\text { Befriedigung }\end{array}$ & Vergnügen, das Leben genießen \\
\hline Leistung & $\begin{array}{l}\text { Persönlicher Erfolg gemäß } \\
\text { den sozialen Standards }\end{array}$ & $\begin{array}{l}\text { Ambitionen, Einfluss, Können, } \\
\text { Erfolg, Intelligenz, } \\
\text { Selbstrespekt }\end{array}$ \\
\hline Macht & $\begin{array}{l}\text { Sozialer Status, Dominanz } \\
\text { über Menschen und } \\
\text { Ressourcen, Prestige }\end{array}$ & $\begin{array}{l}\text { Soziale Macht, Besitz, Autorität, } \\
\text { das Gesicht in der Öffentlichkeit } \\
\text { wahren, soziale Anerkennung }\end{array}$ \\
\hline Sicherheit & $\begin{array}{l}\text { Sicherheit und Stabilität der } \\
\text { Gesellschaft, der Beziehung } \\
\text { und des eigenen Selbst, } \\
\text { Harmonie }\end{array}$ & $\begin{array}{l}\text { Nationale Sicherheit, } \\
\text { Reziprozität von erwiesenen } \\
\text { Gefallen, familiäre Sicherheit, } \\
\text { Zugehörigkeitsgefühl }\end{array}$ \\
\hline Konformität & $\begin{array}{l}\text { Unterdrückung von } \\
\text { Handlungen und Aktionen, } \\
\text { die andere verletzen und } \\
\text { soziale Erwartungen } \\
\text { erzwingen }\end{array}$ & $\begin{array}{l}\text { Eltern und ältere Leute in Ehren } \\
\text { halten, Gehorsam, } \\
\text { Selbstdisziplin, Höflichkeit, }\end{array}$ \\
\hline Tradition & $\begin{array}{l}\text { Respekt und Verpflichtung } \\
\text { gegenüber den kulturellen } \\
\text { oder religiösen Bräuchen } \\
\text { und Ideen }\end{array}$ & $\begin{array}{l}\text { Tradition respektieren, Hingabe, } \\
\text { Mäßigkeit, meine Stellung im } \\
\text { Leben akzeptieren, } \\
\text { Bescheidenheit, }\end{array}$ \\
\hline Benevolenz & $\begin{array}{l}\text { Erhaltung und Förderung } \\
\text { des Wohlergehens von } \\
\text { nahestehenden Menschen }\end{array}$ & $\begin{array}{l}\text { Hilfsbereitschaft, } \\
\text { Verantwortungsbewusstsein, } \\
\text { Vergebung, Ehrlichkeit, } \\
\text { Loyalität, reife Liebe, Treue, } \\
\text { Freundschaft }\end{array}$ \\
\hline
\end{tabular}

Quelle: Mohler, Wohn 2005; Schmidt et al.2007; Schwartz 2012:5ff 


\subsection{Der Wertekreis nach Schwartz}

Aus diesen Wertetypen entwickelte der amerikanisch-israelische Sozialpsychologe Shalom Schwartz den Wertekreis, welcher in dieser Form erstmals 1992 publiziert wurde. Der Wertekreis besteht aus den zehn Wertetypen, welche kreisförmig angeordnet werden. Die kreisförmige Anordnung der Werte stellt das Schlüsselkonzept des Wertekreises dar (Bardi, Schwartz 2003). Wertetypen, die sich ähnlich sind, liegen im Kreisdiagramm nah beieinander, wohingegen sich konfligierende Wertetypen gegenüberstehen (Mohler, Wohn 2005). Das Ausleben von bestimmten Werten hat psychologische, praktische und soziale Auswirkungen, die im Einklang oder im Konflikt mit anderen Werten stehen (Bardi, Schwartz 2003). Angewendet auf den Wertekreis bedeutet das: Je näher sich zwei Werte im Wertekreis stehen, desto ähnlicher ist die zu Grunde liegende Motivation hinter diesen Werten. Je mehr Distanz zwischen den Werten im Wertekreis liegt desto mehr unterscheidet sich die Motivation (Schwartz 2012:10). 


\section{Abbildung 1: Der Wertekreis nach Schwartz}

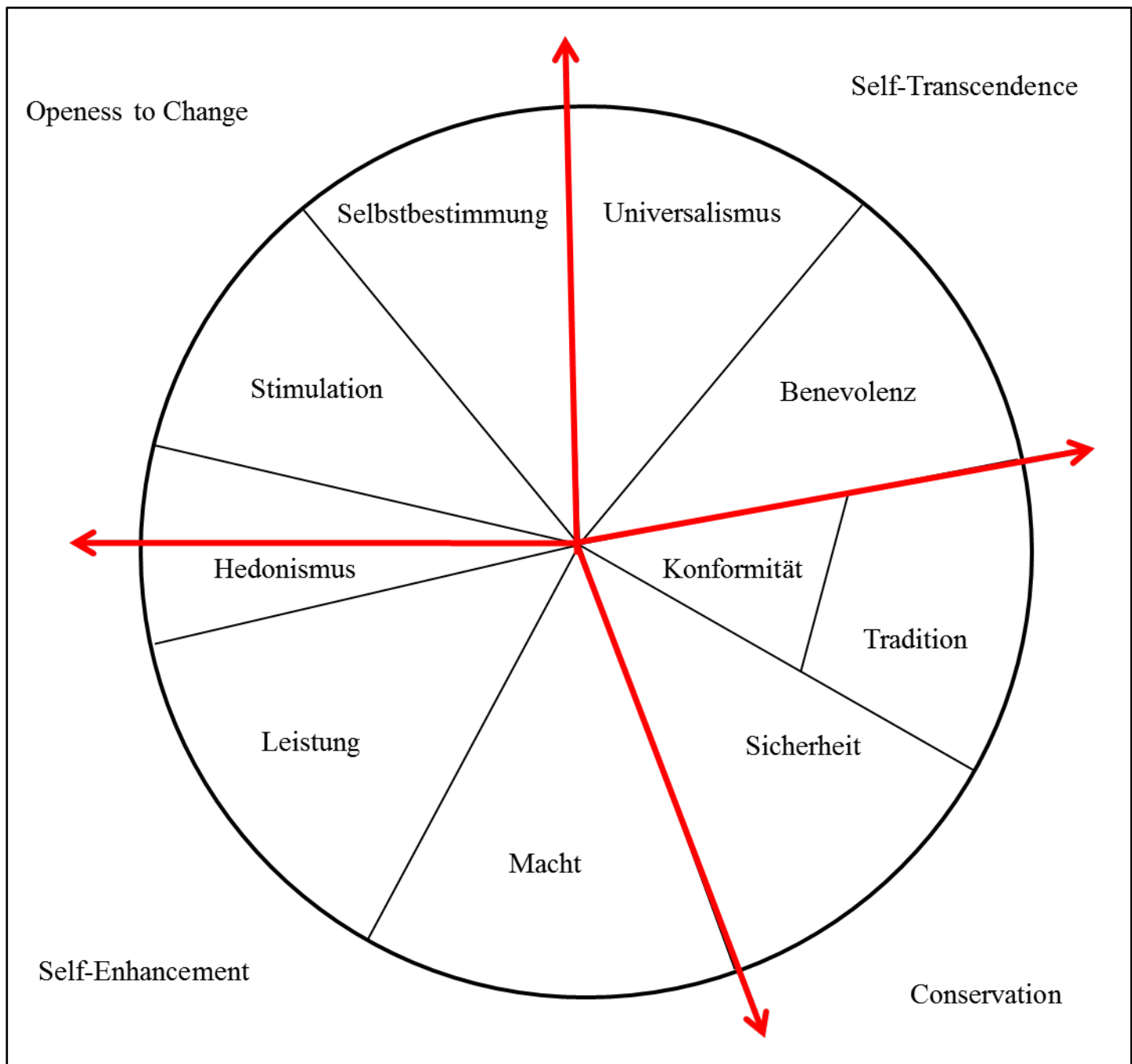

Quelle: Schwartz, Sagie 2000:470

In einem zweiten Schritt werden die zehn Wertetypen auf zwei bipolaren Dimensionen, welche durch vier Wertetypen höherer Dimension erklärt werden, organisiert. In Abbildung 1 wird dies durch die rot eingefärbten Achsen dargestellt. Die Wertetypen „Openess to Change“ und „Conservation“ spannen die erste Dimension auf. Hier stehen Werte wie unabhängiges Denken, Freiheit und Kreativität Werten wie Gehorsam, Selbstdisziplin, Tradition und Sicherheit gegenüber. Die zweite Dimension wird von den Wertetypen höherer Ordnung „SelfEnhancement“ und „Self-Transcendence“ aufgespannt, hier stehen auf der einen Seite Werte wie Erfolg, Macht und Anerkennung und auf der anderen Seite Werte wie Toleranz, Loyalität und Weltoffenheit. 
Die zehn Wertetypen werden in dem 56-Items langen „Schwartz Value Survey“ (SVS) abgefragt und überprüft. Die Fragen des SVS sind so ausgelegt, dass der Befragte beispielhaft eine Person beschrieben bekommt und dann auf einer Skala einschätzen soll, wie ähnlich oder unähnlich ihm diese Person ist (Schwartz 2012).

Zwischen 1988 und 1992 wurde durch den SVS in 18 Studien in 38 Nationen empirisch überprüft, ob die 10 Wertetypen in unterschiedlichen Kulturen identifiziert werden können (Schwartz 1992; Schwartz 1994). Die Ergebnisse der Studien konnten die Annahme gleicher Wertetypen in unterschiedlichen Kulturen bestätigen, wodurch die Wertetheorie von Schwartz als universell gültig anerkannt wurde (Schwartz 1992; Schwartz 1994:118). Inzwischen bauen viele Studien von Schwartz auf seiner Wertetheorie auf, wodurch sie sich zu einer der bedeutendsten Theorien der aktuellen Werteforschung entwickelt hat (Schwartz 1992; Bilsky, Schwartz 1994; Schwartz, Sagie 2000; Schwartz, Bardi 2001; Schwartz et al. 2001; Bardi, Schwartz 2003; Schmidt et al. 2007).

Aus dem SVS wurde 2005 der PVQ (Portraits Value Questionnaire) entwickelt. Er war ursprünglich für die Befragung von Kindern gedacht, ist jedoch genauso bei Erwachsenen anwendbar. Den PVQ gibt es in zwei Versionen, mit 40 oder mit 21 Items. Der PVQ-21 wurde speziell für längere Fragebögen konzipiert um die Befragungsdauer kurz zu halten. Obwohl durch die Kürzung des SVS die Reliabilität der Messung komprimiert wurde, liefert der PVQ-21 eine ausreichende Validität der Ergebnisse (Davidov et al. 2008). Der PVQ-21 wird beispielsweise im European Social Survey (ESS) abgefragt und ist Teil einiger Studien in der Werteforschung (Mohler, Wohn 2005; Cieciuch, Davidov 2012).

Mohler und Wohn konnten 2005 die von Schwartz entwickelte Struktur des Wertekreises nicht replizieren. Einige Wertetypen konnten gar nicht oder an einer anderen Position im Wertekreis nachgewiesen werden. Dieses abweichende Ergebnis wurde jedoch von Strack et al. 2008 widerlegt. Sie bestätigen, dass sich der Wertekreis mit Hilfe des PVQ-21 operationalisieren lässt (Strack et al. 2008:100). 


\subsection{Berufe im Wertekreis}

Wenn Gruppen aufgrund von gemeinsamen Merkmalen zusammengefasst werden, wird in der Sozialpsychologie von sozialen Kategorien gesprochen (Pendry 2007:116; Klauer 2008:23). Innerhalb dieser Kategorien können sich Stereotype entwickeln. Unter Stereotypen versteht Eckes:

„kognitive Strukturen, die sozial geteiltes Wissen über die charakteristischen Merkmale von Angehörigen sozialer Kategorien enthalten.“ (Eckes 2008:92).

Diese Überzeugungen über Attribute einer Gruppe können allen Mitgliedern der Gruppe gleichermaßen zugeschrieben werden (Aschmore, Del Boca 1981).

Nach Knafo und Sagiv (2004) und Strack (2010) können verschiedene Berufsgruppen an unterschiedlichen Positionen im Wertekreis angeordnet werden, da Werte einen entscheidenden Einfluss auf berufliches Verhalten ausüben (Knafo, Sagiv 2004:255).

Individuen schließen sich Gruppen an, die kompatibel mit dem eigenen Charakter sind und die es erlauben, die eigene Persönlichkeit zu entfalten und gesteckte Ziele zu erreichen. Auch Studierende wählen das jeweilige Hauptfach entsprechend ihrer Interessen, Fähigkeiten und Motivation aus, wobei die Motivation und damit die Hierarchie der dahinterstehenden Werte auch angepasst werden können (Knafo, Sagiv 2004:256). 


\section{Abbildung 2: Berufsgruppen im Wertekreis}

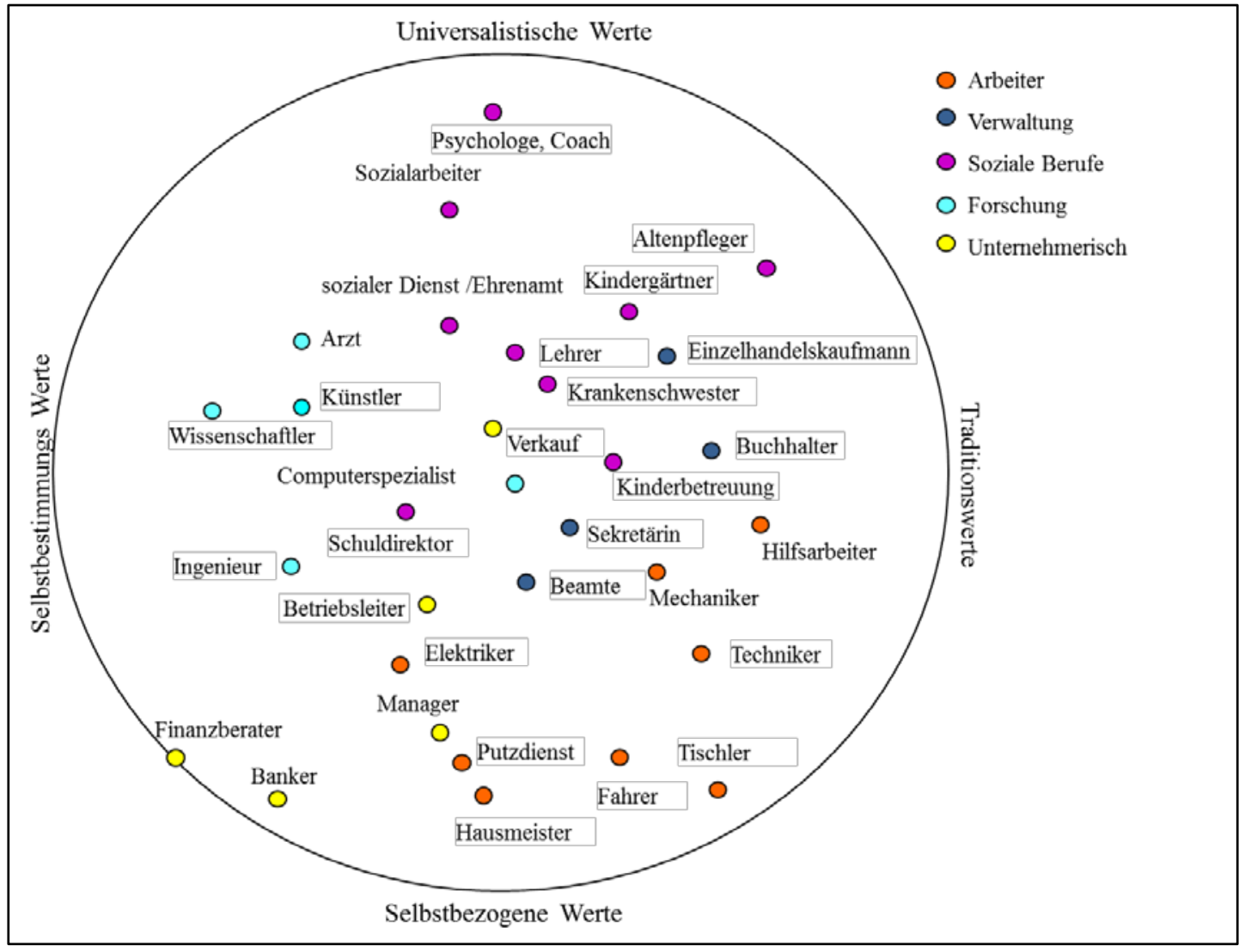

Quelle: Eigene Darstellung nach Strack, Boos 2010 und Knafo, Sagiv 2004:263

Anhand der Abbildung 2 wird deutlich, dass ähnliche Berufsbilder innerhalb des Wertekreises nah zueinander angeordnet werden können. Vergleichbar mit der Interpretation des Wertekreises haben diese nah beieinander stehenden Berufsgruppen ähnliche Werte wohingegen distanziertere Berufsgruppen gegensätzliche Wertvorstellungen aufweisen.

Strack identifiziert in Abbildung 2 fünf Berufsgruppen: Arbeiter (Hausmeister, Tischler, Mechaniker) können im Wertekreis zwischen Traditionswerten und selbstbezogenen Werten lokalisiert werden, Verwaltungsberufe (Sekretärin, Beamte) bei Traditionswerten, soziale Berufe (Psychologe, Lehrer) zwischen Traditionswerten und universalistischen Werten, Forschung (Arzt, Wissenschaftler) bei Selbstbestimmungswerten und die unternehmerische Berufsgruppe (Banker, Manager) zwischen Selbstbestimmungswerten und selbstbezogenen Werten. 


\section{Material und Methoden}

\subsection{Forschungsziel}

Im Rahmen der Studie soll untersucht werden, ob sich die Werte in unterschiedlichen Studienfächern voneinander unterscheiden und inwiefern ein Unterschied der Werte zwischen Studienanfängern (Bachelor, 1. Staatsexamen und Vordiplom) und Fortgeschrittenen (Master, 2. Staatsexamen, Diplom und Promotion) erkennbar ist.

Bei der Positionierung der Fachdisziplinen im Wertekreis wird erwartet, dass sich ökologische Agrarwissenschaften, Landschaftsökologie und Biologie in den Wertetypen Universalismus und Selbstbestimmung wiederfinden, da sie sich stark mit dem Schutz und dem Respekt vor der Natur auseinandersetzen. In Anlehnung an die Ergebnisse von Strack wird Psychologie im Wertetyp Universalismus und BWL/VWL zwischen den Wertetypen Hedonismus, Leistung und Macht erwartet. Konventionelle Agrarwissenschaftler werden zwischen BWL/VWLern und ökologischen Agrarwissenschaftlern in den Wertetypen Universalismus und Selbstbestimmung vermutet, da sie sich einerseits mit ökonomischen Aspekten und anderseits mit der schützenswerten Natur befassen. Juristen werden als sehr traditionell und konservativ eingeschätzt, aus diesem Grund werden sie in den Wertetypen Tradition und Konformität erwartet.

Des Weiteren soll analysiert werden, ob Studienfächer mit ähnlichen Wertvorstellungen auch Parallelen hinsichtlich ihrer Einstellungen zur landwirtschaftlichen Nutztierhaltung aufweisen.

\subsection{Fragebogenkonstruktion und Datenerhebung}

Die Umfrage fand unter Studierenden unterschiedlicher Fachdisziplinen statt. Es wurde bewusst darauf verzichtet die Umfrage unter Konsumenten im Lebensmitteleinzelhandel durchzuführen, da hier zu viele unterschiedliche Branchen aufeinandertreffen, sodass das herausarbeiten von Gemeinsamkeiten und Unterschieden erschwert wäre. In Anlehnung an die Charakteristika des Homo oeconomicus $^{10}$ wurden Fragen zur Bewusstseinszuschreibung entwickelt, um so Menschen und Tiere als wirtschaftlich handelnde Subjekte vergleichen und in

\footnotetext{
${ }^{10}$ Homo oeconomicus. Handelt rational unter Knappheit mit einer klaren Bedürfnishierarchie für die maximale Bedürfnisbefriedigung. Restriktionen schränken ihn ein (Marggraf et al. 2005:63).
} 
Beziehung setzen zu können. Des Weiteren wurden die Teilnehmer zu ihrer Zufriedenheit mit der konventionellen landwirtschaftlichen Praxis und ihrer Einstellung zur Relevanz des tierischen Wohlbefindens in Haltungsbedingungen befragt.

Nach der Angabe ihres Studienfachs wurden die Studenten zu ihrer individuellen Identifikation mit ihrem Studienfach befragt. Dazu wurde die vier Item Skala von Doosje et al. (1995) übernommen.

Um die Werte der Befragten zu erfassen wurde der PVQ-21 am Ende des Fragebogens integriert. Nachdem Schmidt et al. bestätigt haben, dass sowohl der SVS als auch der PVQ die gleichen motivationalen Wertetypen messen und somit als gleichwertige Alternativen angesehen werden können (Schmidt et al. 2007:271), wurde gezielt der kürzere PVQ-21 ausgewählt, da dieser extra für sehr lange Fragebögen konzipiert wurde und so die Befragungsdauer beschränkt werden konnte.

Zur Beantwortung des PVQ-21 standen den Befragten die Kategorien „Ist mir überhaupt nicht ähnlich“, „Ist mir nicht ähnlich“, „Ist mir nur ein kleines bisschen ähnlich“, „Ist mir etwas ähnlich“, „Ist mir ähnlich“ und „Ist mir sehr ähnlich“ zur Auswahl. Um die gewonnenen Daten im Wertekreis darstellen zu können, wurden die Items des Wertekreises ipsatiert. Beim Ipsatieren wird der Mittelwert aller 21 PVQ Items des jeweiligen Befragten von jedem Item abgezogen. Dadurch werden die Rohwerte von der individuellen Zustimmungstendenz des Befragten bereinigt (Horowitz et al. 2000:13). Die Gewichtung der ipsatierten Items des PVQ-21 wurde von Dobewall und Strack (2011) übernommen (siehe Anhang 1).

Nach einem Pretest mit 25 unabhängigen Personen wurde der Fragebogen erneut überarbeitet und schließlich zur Befragung freigeschaltet. Innerhalb eines Frageblocks wurden die Fragen randomisiert um Folgeeffekte ausschließen zu können. Die Studie richtete sich hauptsächlich an Studenten, Absolventen und Promovenden, was dem Befragungsmuster von Schwartz nahe kommt (vergl. Schwartz 1994:91). Der Fokus lag dabei auf den Fachrichtungen Agrarwissenschaften, ökologische Agrarwissenschaften, Landschaftsökologie, Biologie, Betriebs- und Volkswirtschaftslehre, Jura, Philosophie sowie Psychologie. Diese Fächer wurden aufgrund ihrer fachlichen Nähe zum Thema Tierhaltung, Fleischproduktion und Landwirtschaft ausgewählt. Grundsätzlich war die Umfrage 
jedoch für jeden Interessenten offen. So sollte erreicht werden, dass Daten aus den verschiedenen Einflussbereichen auf die landwirtschaftliche Erzeugung mit in die Befragung einflossen. Es wurde darauf geachtet, dass aus jeder Fachgruppe ungefähr gleich viele Probanden befragt wurden. Dies stellte sich vor allem für die Fachbereiche Betriebs- und Volkswirtschaftslehre sowie Philosophie als schwierig heraus, da sich diese Fachbereiche inhaltlich nicht direkt mit landwirtschaftlicher Produktion auseinandersetzen und so das Interesse an der Umfrage eher gering war. Obwohl die Umfrage gezielt an Orten verteilt wurde, an denen die verschiedenen Fachdisziplinen aktiv sind, mussten die Fächer Betriebs- und Volkswirtschaftslehre zusammengefasst werden, um eine ausreichende Gruppengröße vorweisen zu können.

Die Umfrage wurde mit Unipark erstellt, alle Teilnehmer haben online an der Umfrage teilgenommen. Verbreitet wurde sie hauptsächlich über Verteiler von Fachschaften, Semestern und Konferenzen. Des Weiteren wurden OnlinePlattformen genutzt wie beispielsweise das „Schwarze Brett“ von StudIP Göttingen. Der Umfragezeitraum erstreckte sich vom 11.12.2013 bis zum 06.02.2014. Es wurde ein längerer Befragungszeitraum gewählt, da die Weihnachtsfeiertage und der Jahreswechsel in diesen Zeitraum fielen. Die Beendigungsquote des Fragebogens beträgt 45,05 \%. Die gewonnenen Daten wurden unter Verwendung von Predictive Analysis Software (PASW) 22 statistisch ausgewertet.

\subsection{Skalenbildung}

Jede Skala wurde durch mindestens drei Items operationalisiert. Durch eine Reliabilitätsanalyse wurde untersucht, ob die Items zu einer Skala zusammengefasst werden können. Notwendige Voraussetzung dafür ist, dass die Items eine einheitliche Orientierung haben und die inhaltliche Bedeutung von hohen und niedrigen Werten übereinstimmt. Wenn dies nicht der Fall ist, dann müssen einzelne Items umcodiert werden. Zudem müssen sich alle Items sinnvoll zu einer Gesamtskala zusammenfassen lassen (Brosius 2013:823). Fast alle Items des Fragebogens wurden mit Hilfe einer endpunktbenannten Skala von 1 bis 5 beantwortet, dadurch lassen sich die Items problemlos zusammenfassen. Dabei war die Ziffer 1 jeweils mit „Nein, lehne ab“ und die Ziffer 5 mit „Ja, stimme zu“ beschriftet. Der Vorteil einer endpunktbenannten Skala ist, dass diese wie eine 
Intervallskala behandelt werden kann und somit mehr statistische Verfahren zulässt als die Ordinalskala (ipid:479). Durch den einheitlichen Wertebereich der Skala kann zudem ausgeschlossen werden, dass ein Item ein anderes dominiert.

Ausschlaggebend für die Reliabilität der Skalen ist Cronbachs Alpha ( $\alpha$ ). Je stärker die Items, die zusammengefasst werden sollen, miteinander korrelieren, desto höher fällt $\alpha$ aus. Die Anzahl der Items, die für eine Skala herangezogen werden, tragen mit abnehmendem Grenznutzen zur Höhe von Cronbachs Alpha bei (Baur, Fromm 2008:319). Eine Skala gilt ab einem $\alpha$ von 0,7 als reliabel und darf somit zusammengefasst werden (Brosius 2013:826).

Tabelle 2: Reliabilitätsanalyse für die Skalenbildung

Items

Skala

Cronbachs

Alpha

Menschen ...

...verfolgen eigene Interessen.

...verhalten sich vernünftig.

...mögen manches und lehnen manches ab.

...haben einen freien Willen.

...können sich freuen und leiden.

$\begin{array}{ll}\text { Bewusstsein } & 0,705\end{array}$

...haben eine eigene Persönlichkeit.

...können niedrige und hohe Lebensqualität

unterscheiden.

Tiere ...

...verfolgen eigene Interessen.

...verhalten sich vernünftig.

...mögen manches und lehnen manches ab.

...haben einen freien Willen.

...können sich freuen und leiden.

Bewusstsein $\quad 0,755$

...haben eine eigene Persönlichkeit.

...können niedrige und hohe Lebensqualität

unterscheiden.

- Nutztiere empfinden Leid und Schmerz in ähnlicher Form wie Menschen.

- Das Wohlbefinden der Nutztiere hat Einfluss auf deren Lebensqualität.

Tierleid

0,789

- Für Nutztiere spielt das Wohlbefinden eine ähnliche Rolle wie für Menschen. 


\begin{tabular}{|c|c|c|c|}
\hline \multicolumn{2}{|c|}{ Items } & Skala & $\begin{array}{l}\text { Cronbachs } \\
\text { Alpha }\end{array}$ \\
\hline • & $\begin{array}{l}\text { Das Wohlbefinden von Nutztieren ist mir } \\
\text { wichtig. } \\
\text { Vorlieben von Nutztieren sollten bei } \\
\text { Tierschutzentscheidungen berücksichtigt werden. } \\
\text { Ich bin für artgerechte Haltung von Nutztieren. }\end{array}$ & Tierwohl & 0,716 \\
\hline & $\begin{array}{l}\text { Tierhaltung in der konventionellen } \\
\text { Landwirtschaft in Deutschland ist artgerecht. } \\
\text { Das Haltungssystem von landwirtschaftlichen } \\
\text { Nutztieren ist verbesserungswürdig. } \\
\text { Ich halte die EU Vorschriften zu } \\
\text { Haltungsbedingungen von landwirtschaftlichen } \\
\text { Nutztieren für ausreichend. }\end{array}$ & Status Quo & 0,849 \\
\hline & $\begin{array}{l}\text { Wie häufig essen Sie in der Woche Fleisch und } \\
\text { Wurst? } \\
\text { Essen Sie im Vergleich zu anderen Studierenden } \\
\text { wenig oder viel Fleisch? }\end{array}$ & Fleischkonsum & 0,856 \\
\hline & $\begin{array}{l}\text { Ich sehe mich selbst als typisches Mitglied dieses } \\
\text { Studiengangs. } \\
\text { Ich bin froh, dieses Fach gewählt zu haben. } \\
\text { Ich fühle eine starke Bande zu dem Berufsbild } \\
\text { dieses Fachs. } \\
\text { Ich identifiziere mich mit anderen, die dieses } \\
\text { Fach studiert haben. }\end{array}$ & $\begin{array}{l}\text { Identifikation } \\
\text { Studienfach }\end{array}$ & 0,756 \\
\hline
\end{tabular}

Quelle: Eigene Daten

Die Skalen Tierleid und Tierwohl korrelieren stark miteinander, aus diesem Grund wurden sie zur Skala Tierleid und -wohl zusammengefasst $(\alpha=0,853)$. 


\section{Ergebnisse}

\subsection{Beschreibung der Stichprobe}

Im Rahmen der Befragung zur persönlichen Einstellung zur landwirtschaftlichen Nutztierhaltung und der Messung von individuellen Werten konnten 619 beantwortete Fragebögen ausgewertet werden. An der Befragung haben 22,9 \% männliche und 61,9 \% weibliche Studierende teilgenommen, bei 15,2 \% fehlte eine Angabe zum Geschlecht. Damit sind Frauen in dieser Umfrage stärker vertreten als Männer. Deutlich mehr Probanden haben angegeben sich im Bachelor zu befinden als im Master oder der Promotion. Das Durchschnittsalter beträgt 26,28 Jahre $(\mathrm{SD}=5,44)$.

Teilnehmer, die mehr als ein Fach studieren, wurden gebeten sich für ein Hauptfach zu entscheiden. Abbildung 3 stellt die Eingliederung der Teilnehmer in die angegebenen Hauptfächer dar.

\section{Abbildung 3: Häufigkeit der Studienfächer}

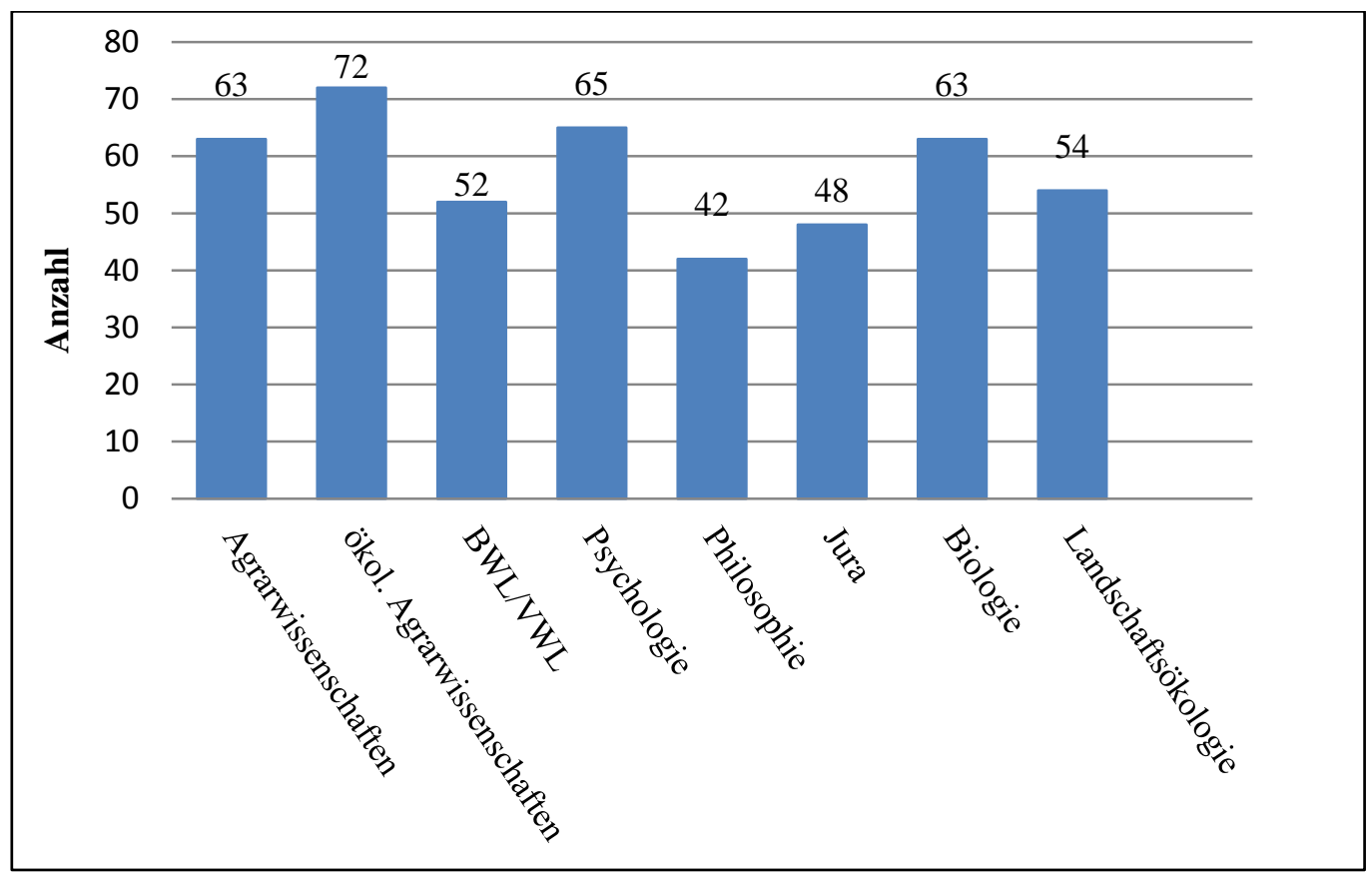

Quelle: Eigene Daten

Die Anzahl der Befragten in den Studierendengruppen ist relativ ausgeglichen. Die Fachrichtungen Philosophie und Jura bilden die jeweils kleinsten Repräsentantengruppen. 
Neben der Fachgruppe wurde ebenfalls abgefragt, wie weit die Befragten in ihrem Studium fortgeschritten sind. 52,2 \% gaben an, sich im Bachelorstudium, Vordiplom oder 1. Staatsexamen zu befinden, 23,1 \% waren im Masterstudium, Diplom oder im 2. Staatsexamen. 7,6 \% hatten bereits ihren Master abgeschlossen und waren im Beruf oder mit ihrer Promotion beschäftigt. Bei 17,1\% fehlt eine Angabe zum Studienabschnitt.

\subsection{Einstellungen zur landwirtschaftlichen Nutztierhaltung in verschiedenen Fachdisziplinen}

Mithilfe einer einfaktoriellen ANOVA wurde untersucht, ob es signifikante Unterschiede in der Zuschreibung von Bewusstsein für Menschen und Tiere zwischen den Fachdisziplinen gibt. Bei der Bewusstseinszuschreibung für Menschen kann kein signifikanter Unterschied festgestellt werden, bei Tieren besteht jedoch ein signifikanter Unterschied zwischen einigen Fachdisziplinen.

Tabelle 3: Signifikante Mittelwertsunterschiede sowie Effektgröße nach Cohen für die "Bewusstseinszuschreibung Tier" in den Studienfächern

\begin{tabular}{llccc} 
Studienfach & & $\begin{array}{c}\text { Mittelwert- } \\
\text { differenz }\end{array}$ & Sig. & $\begin{array}{c}\text { Effekt } \\
\mathbf{d}_{\text {Cohen }}\end{array}$ \\
\hline \hline \multirow{2}{*}{ Agrarwissenschaften } & BWL/VWL & 0,45 & 0,041 & 0,625 \\
\multirow{3}{*}{$\begin{array}{l}\text { okologische } \\
\text { Agrarwissenschaften }\end{array}$} & Landschaftsökologie & $-0,39$ & 0,016 & 0,658 \\
\cline { 2 - 5 } & BWL/VWL & 0,72 & 0,000 & 1,104 \\
BWL/VWL & Jura & 0,49 & 0,007 & 0,741 \\
\cline { 2 - 5 } & Psychologie & $-0,46$ & 0,029 & 0,651 \\
Psychologie & Biologie & $-0,55$ & 0,001 & 0,837 \\
Jura & Landschaftsökologie & $-0,85$ & 0,000 & 1,325 \\
\hline & Landschaftsökologie & $-0,39$ & 0,014 & 0,66 \\
\cline { 2 - 6 } & Landschaftsökologie & $-0,61$ & 0,000 & 0,955 \\
\hline
\end{tabular}

Zur Bewertung der signifikanten Ergebnisse wurde die Effektgröße nach Cohen berechnet. Diese gibt an, ob die identifizierten Mittelwertsunterschiede als schwacher $(d=0,2)$, mittlerer $(d=0,5)$ oder starker $(d=0,8)$ Effekt zu bewerten sind. Demnach stellt die unterschiedliche Bewusstseinszuschreibung für das Tier zwischen den 
Fachdisziplinen ökologische Agrarwissenschaften und BWL/VWL, Landschaftsökologie und BWL/VWL, Biologie und BWL/VWL sowie zwischen Jura und Landschaftsökologie einen starken Effekt dar.

Bei einem Mittelwertevergleich zwischen den verschiedenen Fachdisziplinen (einfaktorielle ANOVA) für die Skalen Status Quo sowie Tierleid und -wohl zeigt sich, dass Agrarwissenschaftler, BWL/VWLer und Juristen signifikant zufriedener mit dem Status Quo der landwirtschaftlichen Nutztierhaltung sind als Landschaftsökologen, ökologische Agrarwissenschaftler und Philosophen. Gleichzeitig sind Agrarwissenschaftlern, BWL/VWLern und Juristen das Wohlbefinden und das wahrgenommene Tierleid signifikant weniger wichtig als Landschaftsökologen, ökologischen Agrarwissenschaftlern und Biologen.

Im Anschluss wurde analysiert, ob ebenfalls Unterschiede zwischen den Fachdisziplinen hinsichtlich des Fleischkonsums identifiziert werden können. Tabelle 4 stellt die deskriptive Statistik der Variablen Fleischkonsum dar.

\section{Tabelle 4: Fleischkonsum in den Studienfächern}

\begin{tabular}{llclc} 
Studienfach & N & Mittelwert & SD & Standardfehler \\
\hline \hline Agrarwissenschaften & 63 & 2,71 & 1,152 & 0,145 \\
ökologische Agrarwissenschaften & 71 & 1,98 & 0,845 & 0,100 \\
BWL/VWL & 52 & 2,90 & 1,283 & 0,178 \\
Psychologie & 65 & 2,26 & 1,253 & 0,155 \\
Philosophie & 42 & 1,67 & 0,992 & 0,153 \\
Jura & 48 & 2,46 & 1,086 & 0,157 \\
Biologie & 63 & 2,01 & 1,032 & 0,130 \\
Landschaftsökologie & 54 & 1,67 & 0,901 & 0,122 \\
\hline Gesamtsumme & 458 & 2,22 & 1,146 & 0,053 \\
\hline \hline
\end{tabular}

Die Ergebnisse zeigen, dass in den Fachdisziplinen BWL/VWL, Agrarwissenschaften und Jura am meisten Fleisch und Wurstwaren konsumiert werden, wohingegen in den Fachdisziplinen Landschaftsökologie, Philosophie und ökologische Agrarwissenschaften vergleichsweise wenig Fleisch konsumiert wird. 
Anhand einer einfaktoriellen ANOVA wurde untersucht, ob signifikante Unterschiede des Fleischkonsums zwischen den Fachdisziplinen festzustellen sind.

Tabelle 5: Signifikante Mittelwertsunterschiede sowie Effektgröße nach Cohen für den Fleischkonsum in Studienfächern

\begin{tabular}{|c|c|c|c|c|}
\hline Studienfach & & $\begin{array}{l}\text { Mittelwert } \\
\text {-differenz }\end{array}$ & Sig. & Effekt d Cohen \\
\hline \multirow{4}{*}{ Agrarwissenschaften } & $\begin{array}{l}\text { ökologische } \\
\text { Agrarwissenschaften }\end{array}$ & 0,73 & 0,002 & 0,73 \\
\hline & Philosophie & 1,05 & 0,000 & 0,97 \\
\hline & Biologie & 0,70 & 0,014 & 0,65 \\
\hline & Landschaftsökologie & 1,05 & 0,000 & 1,01 \\
\hline \multirow{4}{*}{ BWL/VWL } & $\begin{array}{l}\text { ökologische } \\
\text { Agrarwissenschaften }\end{array}$ & 0,92 & 0,001 & 0,88 \\
\hline & Philosophie & 1,237 & 0,000 & 1,08 \\
\hline & Biologie & 0,887 & 0,003 & 0,78 \\
\hline & Landschaftsökologie & 1,237 & 0,000 & 1,13 \\
\hline \multirow{2}{*}{ Jura } & Philosophie & 0,791 & 0,000 & 0,77 \\
\hline & Landschaftsökologie & 0,791 & 0,004 & 0,81 \\
\hline
\end{tabular}

Auffällig ist, dass sich die Fachbereiche Agrarwissenschaften und BWL/VWL signifikant von den Fachdisziplinen ökologische Agrarwissenschaften, Philosophie, Biologie und Landschaftsökologie unterscheiden. Für Jura kann ebenfalls ein signifikanter Unterschied zu Philosophie und Landschaftsökologie identifiziert werden. 


\subsection{Studenten unterschiedlicher Fachbereiche im Wertekreis}

Zunächst wurden die mit Hilfe des PVQ-21 gewonnenen Daten im Wertekreis dargestellt.

\section{Abbildung 4: Positionierung der Stichprobe im Wertekreis}

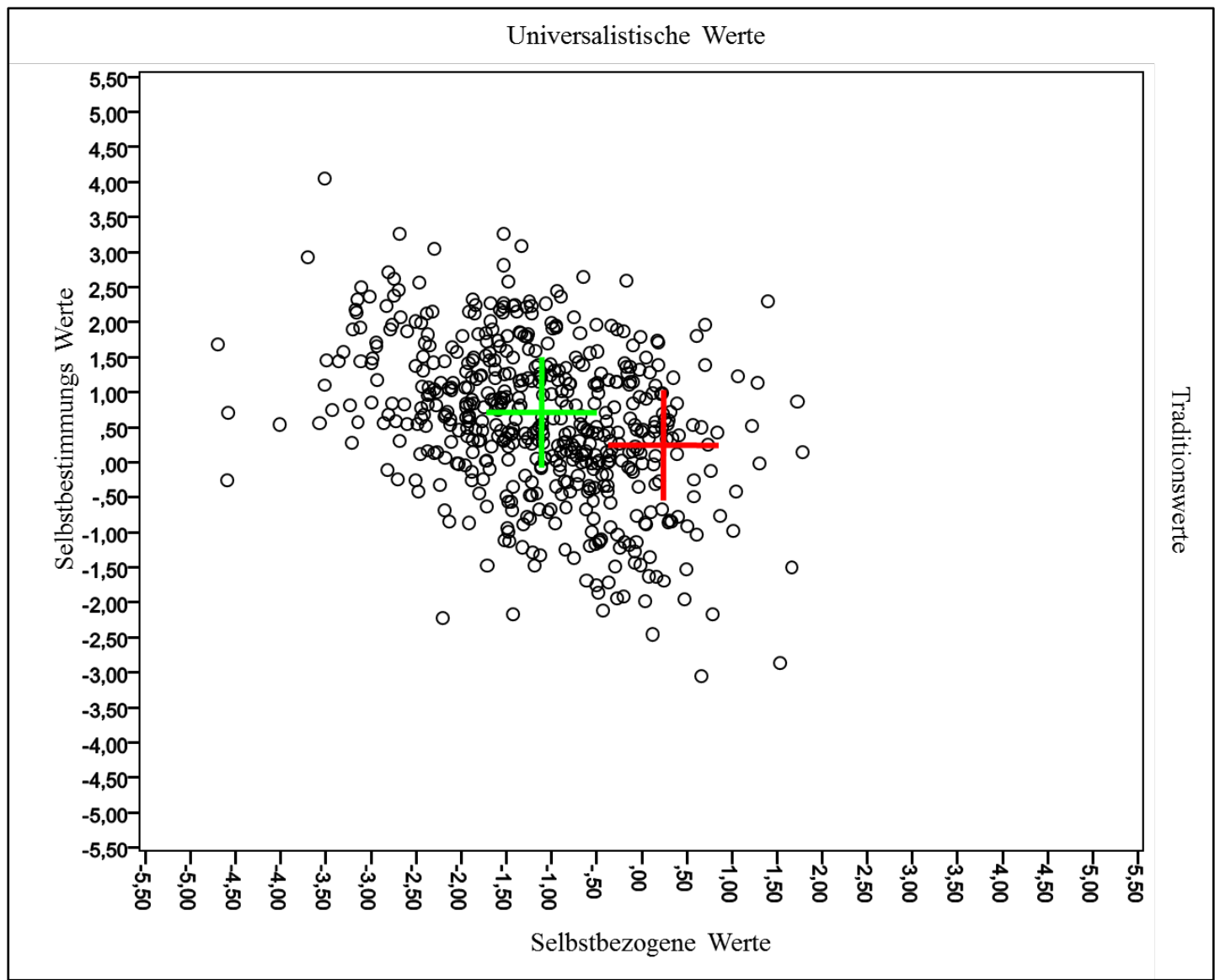

Quelle: Eigene Daten

Der Schnittpunkt des roten Kreuzes stellt den deutschen Mittelwert aus einer Studie von Strack und Dobewall (2012) dar (-0,383/0,364). Der Schnittpunkt des grünen Kreuzes stellt die Mittelwerte der Stichprobe dar ${ }^{11}$. Auffällig ist, dass der Mittelwert der Stichprobe deutlich nach links in Richtung Universalismus und Selbstbestimmung verschoben ist. In Abbildung 5 wird dargestellt, wie sich die einzelnen Studienfächer im Wertekreis positionieren.

11 Universalistische Werte Mittelwert 0,5664 bei einer Standardabweichung von 1,09 bei N 517, Traditionswerte Mittelwert -1.074 bei einer Standardabweichung von 1,074 bei N 517 


\section{Abbildung 5: Studienfächer im Wertekreis}

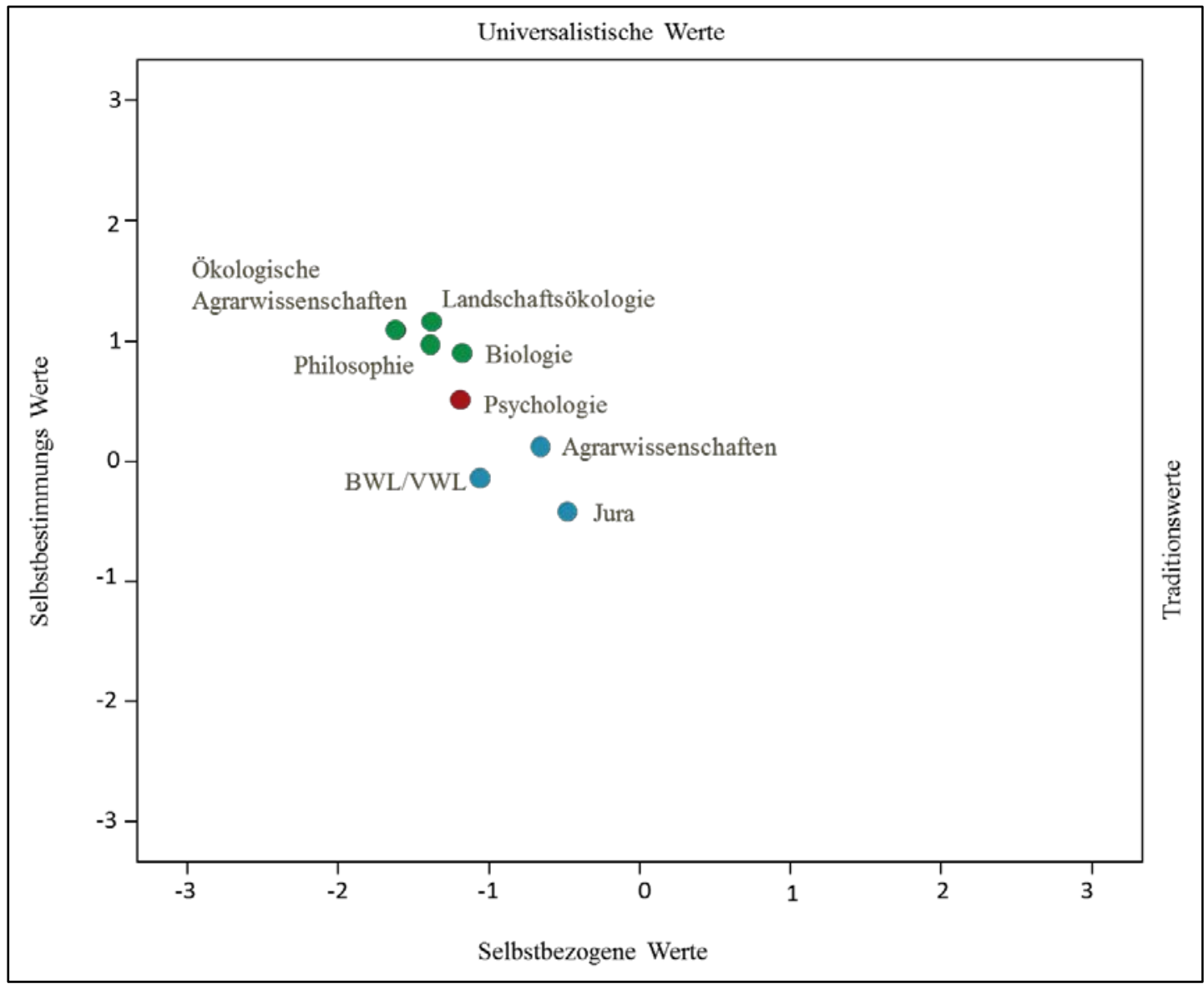

Quelle: Eigene Daten

Es wird deutlich, dass ökologische Agrarwissenschaften, Landschaftsökologie, Philosophie und Biologie im Wertekreis eine andere Position einnehmen als BWL/VWL, Agrarwissenschaften und Jura. Die beschriebene stärkere Betonung der Wertetypen Universalismus und Selbstbestimmung wird durch die Positionierung der Befragten aus den Fachdisziplinen ökologische Agrarwissenschaften, Landschaftsökologie, Philosophie und Biologie hervorgerufen. Besonders auffällig ist, dass sich konventionelle und ökologische Agrarwissenschaftler in der Tendenz gegenüberliegen.

Durch das Allgemeine Lineare Modell wurde untersucht inwiefern das Studienfach, die Studienphase und die Identifikation mit dem Studienfach die Werte aufklären. Die Interaktion der drei genannten Variablen erklären die universalistischen Werte zu $31 \%$ und die Traditionswerte zu $17 \%$. Das Fach hat mit $25 \%$ bei universalistischen Werten und $11 \%$ bei Traditionswerten den meisten Einfluss auf die Werte. 
Abbildung 6: Identifikation mit dem Studienfach konventioneller und ökologischer Agrarwissenschaftler im Wertekreis

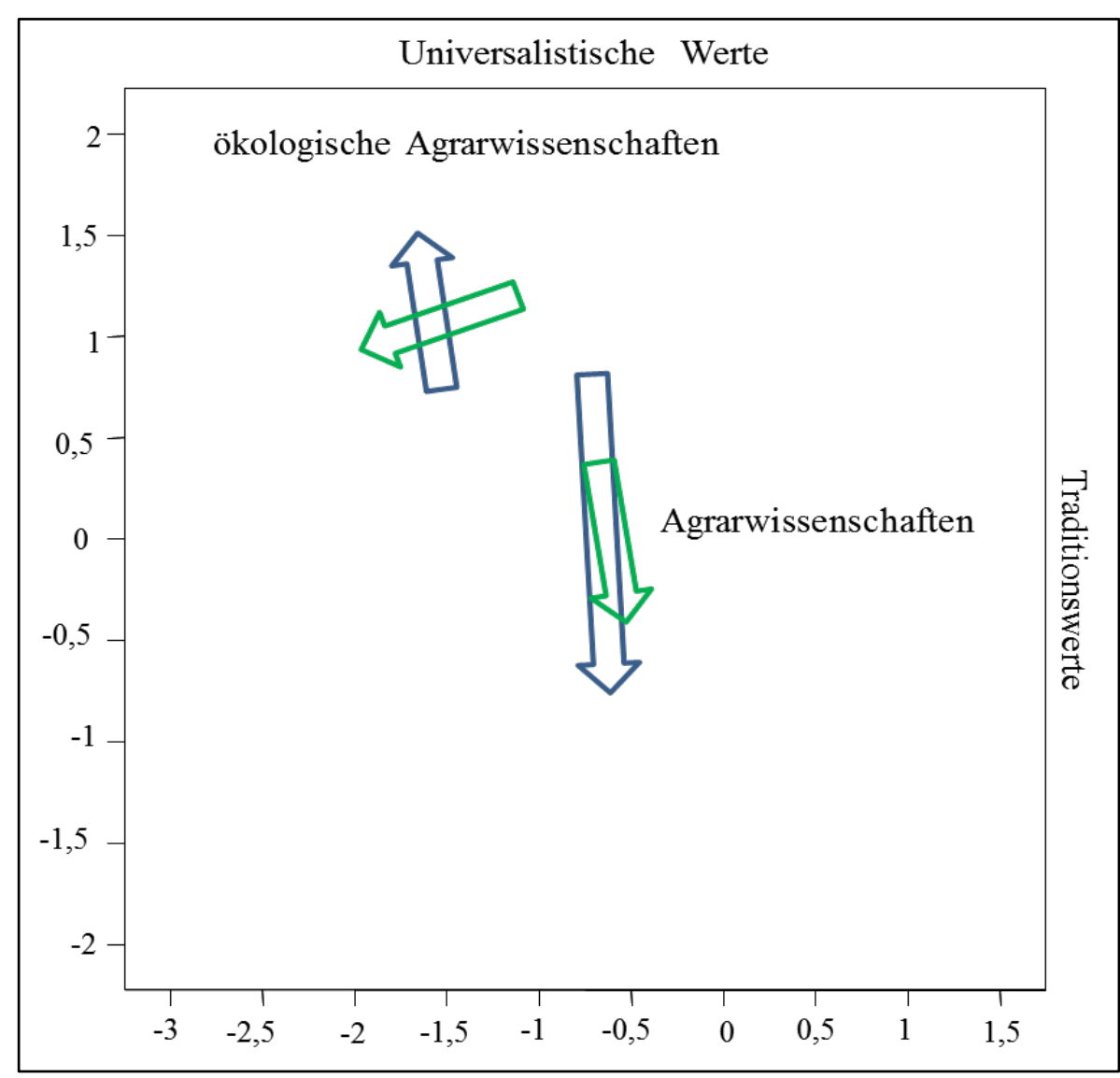

Quelle: Eigene Darstelllung

Die Abbildung 6 verdeutlicht exemplarisch für alle untersuchten Fachdisziplinen die Identifikation mit dem Studienfach für Agrarwissenschaften und ökologische Agrarwissenschaften, aufgeteilt auf die Studienphasen, im Wertekreis. Die blauen Pfeile stellen die Antworten von Studenten aus dem Bachelor, 1. Staatsexamen oder dem Vordiplom dar, die grünen Pfeile symbolisieren die Antworten von Probanden aus dem Master, 2. Staatsexamen, Diplom oder der Promotion. Die Pfeilspitzen verlaufen dabei in Richtung der höchsten Identifikation mit dem Studienfach. Auffällig ist, dass sich die Positionierung der Studierenden aller Disziplinen im Wertekreis von Studienanfängern und -fortgeschrittenen nicht grundlegend unterscheidet. Die Pfeilrichtung verändert sich jedoch in jedem Studiengang außer in Agrarwissenschaften. Am deutlichsten ist dies bei BWL/VWLern, hier verlaufen die Pfeilrichtungen entgegengesetzt. 


\section{Diskussion}

Durch die Studie konnte nachgewiesen werden, dass sich die Fachdisziplinen ökologische Agrarwissenschaften und BWL/VWL, Biologie und BWL/VWL sowie Jura und Landschaftsökologie hinsichtlich der Zuschreibung eines Bewusstseins für Tiere signifikant unterscheiden. Ein ähnliches Bild zeigt sich bei der Zufriedenheit mit den aktuellen Haltungsbedingungen landwirtschaftlicher Nutztiere und der Relevanz tierischen Wohlbefindens für die einzelnen Studienfächer: Agrarwissenschaftler, BWL/VWLer und Juristen sind signifikant zufriedener mit dem Status Quo als Landschaftsökologen, ökologische Agrarwissenschaftler und Philosophen. Gleichzeitig sind Agrarwissenschaftlern, BWL/VWLern und Juristen das Wohlbefinden und das wahrgenommene Tierleid signifikant weniger wichtig als Landschaftsökologen, ökologischen Agrarwissenschaftlern und Biologen. Hinsichtlich des Fleischkonsums bilden sich ähnliche Gruppen: Die Fachbereiche Agrarwissenschaften und BWL/VWL unterschieden sich signifikant von den Fachdisziplinen ökologische Agrarwissenschaften, Philosophie, Biologie und Landschaftsökologie. Bei Jura kann ebenfalls ein signifikanter Unterschied zu Philosophie und Landschaftsökologie identifiziert werden.

Anhand der Ergebnisse können drei Gruppen identifiziert werden, die sich in ihren Werten sehr ähnlich sind. Die erste Gruppe wird durch Landschaftsökologie, ökologische Agrarwissenschaften, Biologie und Philosophie definiert. Sie schreiben Tieren durchschnittlich ein höheres Bewusstsein zu, sind unzufriedener mit dem aktuellen Zustand der landwirtschaftlichen Nutztierhaltung und konsumieren weniger Fleisch als Studierende aus den Fachbereichen BWL/VWL, Agrarwissenschaften und Jura.

Im Wertekreis positionieren sich Landschaftsökologie, ökologische Agrarwissenschaften, Biologie und Philosophie bei den Wertetypen Universalismus und Selbstbestimmung. Hinter dem Wertetyp Universalismus stehen Werte wie Gleichheit, Einheit mit der Natur, Toleranz, soziale Gerechtigkeit sowie eine Welt in Frieden. Der Wertetyp Selbstbestimmung schließt Werte wie Freiheit, Kreativität, Neugierde und Selbstrespekt mit ein. Diese Fachdisziplinen haben demnach im Vergleich zu den Anderen eine hohe Zustimmung zu universalistischen und eine niedrige Zustimmung zu Traditionswerten. Sie sind offen für einen Wandel und halten nicht an Traditionen fest. 
Agrarwissenschaften, BWL/VWL und Jura positionieren sich im Wertekreis zwischen Selbstbestimmung, Stimulation, Hedonismus und Leistung. Der Wertetyp Stimulation umfasst Werte wie ein aufregendes Leben, Wagemut, Abwechslung und sich trauen. Hedonismus umschreibt den Wert „das Leben genießen“ und der Wertetyp Leistung schließt Werte wie Erfolg, Einfluss, Können, Intelligenz und Selbstrespekt ein. Die Fachdisziplinen Agrarwissenschaften, BWL/VWL und Jura sind demnach eher traditionsbewusst und leistungsorientiert, sie wollen an alten Werten festhalten.

Psychologie steht genau zwischen den Ansichten der beiden genannten Gruppen. Dass alle Ergebnisse dieser Studie im Wertekreis oben links angeordnet sind ist nicht überraschend, da in der Umfrage nur Studierende befragt wurden. Diese sind offener für gesellschaftlichen Wandel.

Die Positionierungen der Studienfächer im Wertekreis decken sich ansatzweise mit den Ergebnissen von Strack und den Erwartungen an diese Studie. Die Fächer ökologische Agrarwissenschaften und Landschaftsökologie wurden bei Strack nicht berücksichtigt, sie liegen in dieser Studie zwischen den sozialen Berufen und der Forschung. Diese Position erscheint aufgrund ihrer Themenschwerpunkte logisch, da in den Fachdisziplinen einerseits ein nachhaltiger Umgang mit natürlichen Ressourcen und ein schonender ökologischer Anbau landwirtschaftlicher Produkte im Vordergrund stehen und andererseits viel Forschung auf diesen Gebieten stattfindet. Die Fachbereiche BWL/VWL, Agrarwissenschaften und Jura liegen wie erwartet zwischen der Wissenschaft und den unternehmerischen Berufen. Allerdings wurde vor Beginn der Studie angenommen, dass Juristen noch traditionsbewusster und BWL/VWLer noch leistungs- und machtorientierter sind. Das Ergebnis von BWL und VWL kann durch die Zusammenfassung zu einer Fachdisziplin etwas verfälscht sein, da Betriebs- und Volkswirte teilweise unterschiedliche Einstellungen und Wertvorstellungen vertreten. Der Fachbereich Psychologie liegt bei Strack noch deutlicher in Richtung Universalismus als es in dieser Studie der Fall ist.

Des Weiteren wurde untersucht, ob das fortschreitende Studium einen Einfluss auf die Werte der Befragten hat. Das Ergebnis zeigt, dass sich die Werte in den Studienfächern vom Bachelor zum Master nicht grundlegend ändern. Eine Veränderung in ihrer Ausprägung bzw. Relevanz ist allerdings festzustellen. Dies 
spricht dafür, dass sich die Werte eines Individuums nicht mehr grundlegend ändern, sich die Gewichtungen der einzelnen Werte jedoch verändern können.

Je mehr sich Bachelorstudenten der ökologischen Agrarwissenschaften mit ihrem Studienfach identifizieren, desto mehr Bedeutung haben universalistische Werte für sie. Masterstudenten, die sich stark mit ihrem Studienfach identifizieren tendieren dahingegen $\mathrm{zu}$ universalistischen und Selbstbestimmungswerten. Bei Agrarwissenschaftlern ist keine Veränderung der Wertehierarchie bei Bachelor- und Masterstudenten mit einer hohen Fachidentifikation festzustellen. Dies kann dadurch erklärt werden, dass ein Großteil der Studenten aus einem landwirtschaftlichen Umfeld stammt und sich die dort vorherrschenden und relevanten Werte bereits gefestigt haben. Bei ökologischen Agrarwissenschaftlern ist hingegen bei Studierenden mit starker bzw. schwacher Identifikation eine neue Hierarchiebildung der vorhandenen Werte festzustellen. Dies könnte dadurch erklärt werden, dass zum Teil Studierende mit einem Hintergrund aus konventioneller Landwirtschaft oder ohne landwirtschaftlichen Bezug eingeschrieben sind. Durch die Studieninhalte findet während des Studiums eine neue Hierarchiebildung beziehungsweise eine Festigung der Werte, die für eine ökologische Bewirtschaftung von Bedeutung sind, statt.

Da sich die menschlichen Werte während des Erwachsenwerdens nicht mehr grundlegend ändern ist es ratsam, universalistische Werte bei jüngeren Generationen zu verstärken, um so zu einer höheren Wertschätzung der Natur beizutragen und dem Wunsch nach einer artgerechteren Tierhaltung zu entsprechen. An dieser Stelle sind vor allem Universitäten und die Forschung in der Pflicht, die Traditionen in der landwirtschaftlichen Nutztierhaltung neu zu gestalten.

Des Weiteren erscheint es ratsam im Prozess $\mathrm{zu}$ einer verbesserten und artgerechteren Nutztierhaltung möglichst viele verschiedene Fachdisziplinen in die Entscheidungsfindung zu integrieren, da durch die verschiedenen Wertvorstellungen und Einstellungen der unterschiedlichen Disziplinen zur landwirtschaftlichen Nutztierhaltung eine Lösung gefunden werden könnte, die die divergenten Ansprüche von Konsumenten an die landwirtschaftliche Nutztierhaltung besser abdeckt. 


\section{Fazit}

Normen und Werte helfen den Menschen das für die Gesellschaft angemessene Verhalten zu zeigen und so entsprechende Werteprioritäten zu entwickeln. Demnach können die Werte eines Individuums auf die Werte der kulturellen Gesellschaft zurückgeführt werden. Wenn in einer Gesellschaft beispielsweise beruflicher Erfolg, Reichtum und Leistung hoch angesehen sind, so werden die Mitglieder dieser Gesellschaft versuchen diese Ideale zu erfüllen.

In der vorliegenden Studie wurden Studierende der Fachdisziplinen ökologische Agrarwissenschaften, Landschaftsökologie, Biologie, Philosophie, Psychologie, Agrarwissenschaften, BWL/VWL und Jura befragt. Der Schwerpunkt lag dabei auf ihrer Zufriedenheit mit dem Status Quo der landwirtschaftlichen Nutztierhaltung, der Bedeutung des Wohlbefindens von Tieren sowie der Zuschreibung von Bewusstsein für Menschen und Tiere. Des Weiteren wurde der Fleischkonsum der Befragten sowie die Identifikation mit dem Studienfach erfasst. Abschließend wurden mit Hilfe des von Shalom Schwartz entwickelten Portraits Value Questionnaire (PVQ)-21 die Werte der Studierenden erfasst. Aus den gewonnen Daten können einige Interpretationsansätze gewonnen werden.

Im Wertekreis positionieren sich Landschaftsökologie, ökologische Agrarwissenschaften, Biologie und Philosophie bei den Wertetypen Universalismus und Selbstbestimmung. Hinter dem Wertetyp Universalismus stehen Werte wie Gleichheit, Einheit mit der Natur, Toleranz, soziale Gerechtigkeit sowie eine Welt in Frieden. Der Wertetyp Selbstbestimmung schließt Werte wie Freiheit, Kreativität, Neugierde und Selbstrespekt mit ein. Diese Fachdisziplinen haben demnach im Vergleich zu den Anderen eine hohe Zustimmung zu universalistischen und eine niedrige Zustimmung zu Traditionswerten. Sie sind offen für einen Wandel und halten nicht an Traditionen fest. Sie zeichnen sich durch eine hohe Zuschreibung von Bewusstsein für Tiere, einer unzufriedeneren Einstellung gegenüber den aktuellen Bedingungen der landwirtschaftlichen Nutzierhaltung sowie durch einen geringeren Fleischkonsum aus.

Dem gegenüber positionieren sich die Fachdisziplinen Agrarwissenschaften, BWL/VWL und Jura im Wertekreis zwischen den Wertetypen Selbstbestimmung, Stimulation, Hedonismus und Leistung. Der Wertetyp Stimulation umfasst Werte 
wie ein aufregendes Leben, Wagemut, Abwechslung und sich trauen. Hedonismus umschreibt den Wert „das Leben genießen“ und der Wertetyp Leistung schließt Werte wie Erfolg, Einfluss, Können, Intelligenz und Selbstrespekt ein. Die Fachdisziplinen Agrarwissenschaften, BWL/VWL und Jura sind demnach eher traditionsbewusst und leistungsorientiert, sie wollen an alten Werten festhalten. Studierende dieser Fachdisziplinen schreiben Tieren ein geringeres Bewusstsein zu, sie sind zufriedener mit den aktuellen Bedingungen landwirtschaftlicher Nutztierhaltung und konsumieren mehr Fleisch.

Da sich die menschlichen Werte während des Erwachsenwerdens nicht mehr grundlegend ändern ist es ratsam, universalistische Werte bei jüngeren Generationen zu verstärken, um so zu einer höheren Wertschätzung der Natur beizutragen und dem Wunsch nach einer artgerechteren Tierhaltung zu entsprechen. An dieser Stelle sind vor allem Universitäten und die Forschung in der Pflicht, die Traditionen in der landwirtschaftlichen Nutztierhaltung neu zu gestalten. 


\section{Literaturverzeichnis}

Ashmore, R., Del Boca, F. 1981: Conceptual approaches to stereotypes and stereotyping, in: Cognitive Process in Stereotyping and Intergroup behavior, Hrg.: Hamilton, L., S. 1-35

Bardi, A., Schwartz, S. 2003: Values and Behavior: Strength and structure of relations, Personality and Social Psychology Bulletin, Vol. 29, S. 1207-1220

Baur, N., Fromm, S. 2008: Datenanalyse mit SPSS für Fortgeschrittene, Ein Arbeitsbuch, 2. Auflage, Verlag für Sozialwissenschaften, Wiesbaden

Bilsky, W., Schwartz, S. 1994: Values and Personality, European Journal of Personality, Vol. 8, Issue 3, S. 163-181

Brosius, F. 2013: SPSS 21, mitp Verlag, Heidelberg, München

Cieciuch, J., Davidov, E. 2012: A comparison of the invariance properties of the PVQ-40 and the PVQ-21 to measure human values across German and Polish Samples, Survey Research Methods, Vol. 6, S. 37-48

Covey, S., Merrill, R. 2009: Schnelligkeit durch Vertrauen - Die unterschätzte ökonomische Macht, Offenbach, GABAL Verlag GmbH.

Deutscher Bauernverband 2011: Erlösanteil der Landwirte bei Nahrungsmitteln sinkt, URL: http://www.bauernverband.de/erloesanteil-landwirtenahrungsmitteln-sinkt, Abrufdatum: 12.11.2014

Dobewall, H., Strack, M. 2011: Cultural value differences, value stereotypes, and diverging identities in intergroup conflicts: The Estonian Example, International Journal of Conflict and Violance, Vol. 5, Issue 1, S. 211-223

Dobewall, H., Strack, M. 2012: The value structure in socioeconomically less developed European countries still remains an ellipse, Europe's Journal of Psychology, Vol. 8, Issue 4, S. 587-602

Doosje, B., Ellemers, N., Spears, R. 1995: Perceived intragroup variability as a function of group status and identification, Journal of Experimental Social Psychology, Vol. 31, S. 410-436 
Eckes, T. 2008: Messung von Stereotypen, in: Stereotype, Vorurteile und soziale Diskriminierung, Theorien, Befunde und Interventionen, Hrg.: Pertersen, L., Six, B., Weinheim, Basel, S. 97-106

Horowitz, L., Strauß, B., Kordy, H. 2000: Inventar zur Erfassung interpersonaler Probleme, Deutsche Version, 2. Auflage, Beltz Test GmbH, Göttingen

Klauer, K. 2008: Soziale Kategorisierung und Stereotypisierung, in: Stereotype, Vorurteile und soziale Diskriminierung, Theorien, Befunde und Interventionen, Hrg.: Pertersen, L., Six, B., S. 88-96, Weinheim, Basel

Knafo, A., Sagiv, L. 2004: Values and work environment: Mapping 32 occupations, European Journal of Psychology and Education, Vol. 19, S. 255-273

Marggraf, R., Bräuer, I., Fischer, A., Menzel, S., Stratmann, U., Suhr, A. 2005: Ökonomische Bewertung bei umweltrelevanten Entscheidungen, Einsatzmöglichkeiten von Zahlungsbereitschaftsanalysen in Politik und Verwaltung, Ökologie und Wirtschaftsforschung, Band 55, Metropolis Verlag, Marburg

Mohler, P., Wohn. K. 2005: Persönliche Wertorientierungen im European Social Survey, ZUMA Arbeitsbericht Nr. 2005/01

Pendry, L. 2007: Soziale Kognition, in: Sozialpsychologie eine Einführung, Hrg.: Jonas, K., Stroebe, W., Hewstone, M., S. 111-145, Heidelberg, Springer

Scherr, A. 2013: Normen und Werte, in Spoziologische Basics, S. 271-278, Springer

Schmidt, P., Bamberg, S., Davidov, E., Herrmann, J., Schwartz, S. 2007: Die Messung von Werten mit dem „Portraits Value Questionnaire“, Zeitschrift für Sozialpsychologie, Vol. 38, Issue 4, S. 261-275

Schneider, B. 1987: The people make the place, Personell Psychology, Vol. 40, S. 437-453

Schwartz, S. 1992; Universals in the content and structure of values: Theory and empirical tests in 20 countries, in: Advances in experimental social psychology, Hrg.: Zanna, M., S. 1-65, New York Academic Press

Schwartz, S. 1994: Beyond Individualism/ Collectivism, in: Kim, K., Triandis, H., Kâğitçibaşi, Ç., Choi, S., Yoou, G. Individualism and collectivism- Theory, 
Method and Applications, 18. Auflage, Cross-Cultural Research and Methodology Series

Schwartz, S. 1999: A theory of cultural values and some implications for work, Applied Psychology: An international Review, Vol. 48, Issue 1

Schwartz, S. 2012: An overview of the Schwartz theory of basic values, Psychology and Culture, Vol. 2, Issue 1

Schwartz, S., Bardi, A. 2001: Value Hierarchies across cultures: Taking a Similarities perspective, Journal of Cross-cultural Psychology, Vol. 32, S. 465497

Schwartz, S., Melech, G., Lehmann, A., Burgess, S., Harris, M., Owens, V. 2001: Extending the cross-cultural validity of the theory of basic human values with a different method of measurement, Journal of Cross-cultural Psychology, Vol. 32, S. 519-542

Schwartz, S., Sagie, G. 2000: Value Consensus and Importance: A cross-National Study, Journal of Cross-cultural Psychology, Vol. 31, S. 465-497

Statistisches Bundesamt 2014: Anteil der Ausgaben der privaten Haushalte in Deutschland für Nahrungsmittel, Getränke und Tabakwaren an den Konsumausgaben in den Jahren 1850 bis 2013, URL: http://de.statista.com/statistik/daten/studie/75719/umfrage/ausgaben-fuernahrungsmittel-in-deutschland-seit-1900/, Abrufdatum: 12.11.2014

Strack, M., Boos, M. 2010: Werte psychologisch oder „Wo ist das Grüne im Wertekreis?“ Ringvorlesung „Biodiversität und Gesellschaft“, Göttinger Graduiertenkolleg Gesellschaftswissenschaften, 29. November 2010

Strack, M., Gennerich, C., Hopf, N. 2008: Warum Werte? in: Sozialpsychologie und Werte, Hrsg.: Witte, E., S. 90-130 Lengerich, Pabst

Wiesinger, G. 2005: Landwirtschaft zwischen Tradition und Moderne- Über den Struktur und Wertwandel in der bäuerlichen Lebenswelt, in: Jahrbuch der Österreichischen Gesellschaft für Agrarökonomie, Band 10, S. 165-180 


\section{B. Abschlussdiskussion}

Die landwirtschaftliche Nutztierhaltung steht vor einschneidenden Veränderungen, da die gegenwärtigen Haltungsbedingungen zunehmend von den Konsumenten hinterfragt werden.

Aus ethischer Sicht ist die Frage, ob Tiere von Menschen genutzt werden dürfen oder ob dies moralisch nicht $\mathrm{zu}$ rechtfertigen ist, nicht eindeutig und allgemein $\mathrm{zu}$ beantworten. Unter den Konsumenten scheint die anthropozentrische Sicht und die damit einhergehende Berechtigung das Tier zu menschlichen Zwecken zu nutzen nur von einer Minderheit in Frage gestellt zu werden. 2014 ernährten sich 7,5 \% der deutschen Bevölkerung vegetarisch (Statistisches Bundesamt 2014) und lehnten damit aus ethischen oder gesundheitlichen Gründen die Nutzung von Tieren zur Befriedigung menschlicher Bedürfnisse ab. In der vorliegenden Studie kann die mehrheitlich anthropozentrische Einstellung der Befragten durch eine geringere Zuschreibung von Bewusstsein bei Tieren bestätigt werden.

Aus tierethischer Sicht und aus Sicht der Konsumenten ist eines der elementaren Probleme der landwirtschaftlichen Nutztierhaltung der Umstand, dass Tiere in den Haltungssystemen physisch und psychisch leiden müssen. Die Ergebnisse der 2013 unter Studenten ausgewählter Fachdisziplinen durchgeführten Befragung zeigen, dass insbesondere Studenten aus den Fachdisziplinen ökologische Agrarwissenschaften, Landschaftsökologie, Biologie und Philosophie unzufrieden mit dem aktuellen Zustand der landwirtschaftlichen Nutztierhaltung sind und sie sich artgerechtere Haltungsbedingungen für landwirtschaftliche Nutztiere wünschen.

Der teilweise schlechte Zustand in der Nutztierhaltung muss grundlegend verbessert werden. Eine Möglichkeit bieten hier Präferenz- und Motivationstests. Diese können gezielt dazu eingesetzt werden, die Haltung von Nutztieren mit Hilfe einer direkten Befragung der Tiere zu verbessern, um so Haltungsbedingungen zu entwickeln, in denen die Nutztiere ohne Leidensdruck und Schmerzen gehalten werden können. Studierende verschiedener Fachdisziplinen erachten den Einsatz von Präferenz- und Motivationstests in der Nutztierhaltung als sinnvoll. Es konnten keine fachspezifischen Unterschiede hinsichtlich der eingeschätzten Validität oder der Einstellung zu Präferenz- und Motivationstests festgestellt werden. Mit Hilfe der Studie konnte zudem eine prozentuale Mehrzahlungsbereitschaft von 35,35 \% für 
einen Liter Milch, 21,76 \% für 6 Eier und 20,71 \% für 250 g Hackfleisch aus mit Hilfe von Präferenz- und Motivationstests optimierten Haltungsbedingungen ermittelt werden.

Der Fleischkonsum der Studenten wird zwar durch den Wunsch nach einer artgerechteren Haltung beeinflusst, den Haupteffekt übt jedoch die Zufriedenheit mit dem Status Quo der landwirtschaftlichen Nutztierhaltung aus. Es wird zudem vermutet, dass der Verzicht auf Fleisch eher durch Verbraucherunsicherheiten aufgrund von Skandalen und Schadstoffbelastungen der Lebensmittel begründet ist als tatsächlich durch den Wunsch nach einer artgerechteren Haltung des Tieres.

Damit langfristig eine Veränderung der Haltungsbedingungen von landwirtschaftlichen Nutztieren erfolgen kann, muss sich die Einstellung der Lebensmittelindustrie, der Agrarindustrie, der Konsumenten und des Bildungssektors zur Ressource Nutztier verändern. Sauerberg und Wierzbitza filterten 2013 aus agrarwissenschaftlicher Literatur sieben Einstellungsmöglichkeiten zu landwirtschaftlichen Nutztieren heraus. Die Texte wurden dahingehend analysiert ob sie das Tier eher als Subjekt oder als Produkt ansehen. Die Autoren kamen zu dem Ergebnis, dass Texte, die das Tier als Subjekt ansehen eher seltener sind und häufig aus dem Bereich der ökologischen Agrarwissenschaften stammen. Basierend auf den Ergebnissen der vorliegenden Studie kann bestätigt werden, dass die Fachdisziplinen ökologische Agrarwissenschaften, Landschaftsökologie, Biologie und Philosophie Tiere eher als Subjekte betrachten. Ihnen gegenüber stehen die Fachdisziplinen Jura, BWL/VWL und Agrarwissenschaften, deren Blickwinkel auf das Tier eher objektbezogen ist. Von diesen Fachdisziplinen wird das Tier hauptsächlich als Ressource zur Fleischerzeugung angesehen. Aus diesem Grund sprechen Studierende dieser Fachdisziplinen dem Tier ein geringeres Bewusstsein zu, sie sind zufriedener mit dem aktuellen Zustand der landwirtschaftlichen Nutztierhaltung und konsumieren mehr Fleisch.

Die Verbesserung der Haltungsstandards für Tiere wird nur dann eine breite gesellschaftliche Akzeptanz finden, wenn die artgerechte Tierhaltung als fest verankerter Wert innerhalb der Gesellschaft Anerkennung erfährt und sich das Bedürfnis zu artgerecht erzeugten Produkten bei den Konsumenten festigt. 
Mit Hilfe des Wertekreises konnte ermittelt werden, dass sich die Fachdisziplinen denen die artgerechte Tierhaltung wichtiger ist bei den Wertetypen Universalismus und Selbstbestimmung wiederfinden. Hinter dem Wertetyp Universalismus stehen Werte wie Gleichheit, Einheit mit der Natur, Toleranz, soziale Gerechtigkeit sowie eine Welt in Frieden. Der Wertetyp Selbstbestimmung schließt Werte wie Freiheit, Kreativität, Neugierde und Selbstrespekt mit ein. Diese Werte müssten im Rahmen der Erziehung und Ausbildung stärker angesprochen werden um in der Gesellschaft ein Umdenken zu erzielen.

Agrarwissenschaften, BWL/VWL und Jura sind traditionsbewusster und leistungsorientierter. Sie wollen eher an alten Werten festhalten. Bezogen auf die landwirtschaftliche Nutztierhaltung müssen sich insbesondere Agrarwissenschaftler von den Konventionen der Nutztierhaltung entfernen und offener für einen Wandel in der Agrarindustrie werden. An dieser Stelle sind vor allem Universitäten und die Forschung in der Pflicht, die Traditionen in der landwirtschaftlichen Nutztierhaltung neu zu gestalten. 


\section{Anhang}

\section{Gewichtung der ipsatierten Items}

\begin{tabular}{lcc} 
Item & $\begin{array}{l}\text { Self-Transcendence } \\
\text { (vs. Self- } \\
\text { Enhancement }\end{array}$ & $\begin{array}{l}\text { Conservation (vs. } \\
\text { Openess to } \\
\text { Change) }\end{array}$ \\
\hline \hline Konstante & $-0,975$ & $-0,245$ \\
$\begin{array}{l}\text { Der Person ist wichtig, neue Ideen zu } \\
\text { entwickeln und kreativ zu sein. Sie macht } \\
\text { Sachen gerne auf ihre eigene originelle Art. }\end{array}$ & $-0,056$ & 0,135 \\
\hline $\begin{array}{l}\text { Dieser Person ist wichtig, reich zu sein. Sie } \\
\text { möchte viel Geld haben und teure Sachen } \\
\text { besitzen. }\end{array}$ & 0,178 & $-0,030$ \\
\hline
\end{tabular}

Diese Person hält für wichtig, dass alle Menschen auf der Welt gleich behandelt werden sollten. Sie glaubt, dass jeder Mensch

$-0,173 \quad 0,026$

im Leben gleiche Chancen haben sollte.

Dieser Person ist wichtig, ihre Fähigkeiten zu zeigen. Sie möchte, dass die Leute bewundern was sie tut.

Dieser Person ist wichtig, in einem sicheren Umfeld zu leben. Sie vermeidet alles, was ihre $\quad 0,33 \quad-0,153$ Sicherheit gefährden könnte.

Diese Person mag Überraschungen und hält immer Ausschau nach neuen Aktivitäten. Sie denkt, dass im Leben Abwechslung wichtig ist.

Diese Person glaubt, dass die Menschen tun sollten, was man Ihnen sagt. Sie denkt, dass Menschen sich an Regeln halten sollten, selbst dann wenn es niemand sieht.

Dieser Person ist wichtig, Menschen zuzuhören, die anders sind als sie. Auch wenn sie anderer Meinung ist als andere, will sie die $-0,216 \quad 0,070$ anderen trotzdem verstehen.

Dieser Person ist wichtig, zurückhaltend und bescheiden zu sein. Sie versucht, die Aufmerksamkeit nicht auf sich zu lenken.

Dieser Person ist wichtig Spaß zu haben. Sie gönnt sich selbst gerne was.

$\begin{array}{ll}-0,24 & 0,148\end{array}$

Dieser Person ist wichtig, selbst zu entscheiden, was sie tut. Sie ist gerne frei und unabhängig von anderen.

Dieser Person ist es sehr wichtig, den Menschen um sie herum zu helfen. Sie will

$-0,102 \quad-0,057$

$0,001 \quad 0,138$


für deren Wohl sorgen.

Dieser Person ist wichtig, sehr erfolgreich zu sein. Sie hofft, dass die Leute ihre Leistung 0,182 $-0,017$ anerkennen.

Dieser Person ist wichtig, dass der Staat ihre persönliche Sicherheit vor allen Bedrohungen gewährleistet. Sie will einen starken Staat, der $0,016 \quad-0,150$ seine Bürger verteidigt.

Diese Person sucht das Abenteuer und geht gerne Risiken ein. Sie will ein aufregendes Leben haben.

$0,047 \quad 0,113$

Dieser Person ist wichtig, sich jederzeit korrekt zu verhalten. Sie vermeidet es, Dinge zu tun, die andere Leute für falsch halten $-0,008 \quad-0,156$ könnten.

Dieser Person ist wichtig, dass andere sie respektieren. Sie will, dass die Leute tun, was sie sagt.

Dieser Person ist wichtig, ihren Freunden gegenüber loyal zu sein. Sie will sich für Menschen einsetzen, die ihr nahe stehen.

Diese Person ist fest davon überzeugt, dass die Menschen sich um die Natur kümmern $-0,179$ $-0,015$ sollten. Umweltschutz ist ihr wichtig.

Dieser Person ist Tradition wichtig. Sie versucht, sich an die Sitten und Gebräuche zu halten, die ihr von ihrer Religion oder ihrer

Familie überliefert wurden.

Diese Person lässt keine Gelegenheit aus, Spaß zu haben. Es ist ihr wichtig Dinge zu tun, die ihr Vergnügen bereiten.

$-0,232 \quad 0,053$

\section{Fragebogen}

Quelle: Dobewall, Strack 2011 


\section{Liebe Teilnehmer!}

Ich freue mich sehr, dass Sie an meiner Befragung zum Umgang mit Tieren teilnehmen.

Unter dem Begriff "Nutztiere" werden in dieser Umfrage alle landwirtschaftlich genutzten Tiere (Schwein, Rind, Huhn) verstanden. Haustiere (Hund, Katze) und Wildtiere (Rehe) zählen nicht dazu.

Bevor Sie die Fragen beantworten bitte ich Sie, sich jeweils die kurzen Erläuterungstexte vor den Frageblöcken durchzulesen.

Die Umfrage ist anonym, alle Angaben werden vertraulich behandelt, zusammengefasst und nur in dieser statistisch aufgearbeiteten Form im Rahmen meiner Dissertation veröffentlicht

Sie helfen mir durch ehrliche Antworten

\section{Vielen Dank!}

Kira Greving

Um das Bewusstsein von Mensch und Tier herrschen aktive Diskussionen. Welche Aussagen treffen Ihrer Meinung nach zu? Menschen....

\begin{tabular}{|c|c|c|c|c|c|}
\hline & $\begin{array}{c}\text { Nein, } \\
\text { lehne ab } \\
1 \\
\end{array}$ & 2 & 3 & 4 & $\begin{array}{l}\text { stimme } \\
\mathrm{zu} \\
5 \\
\end{array}$ \\
\hline ... verfolgen eigene Interessen. & 0 & 0 & 0 & 0 & 0 \\
\hline ... verhalten sich vernünftig. & 0 & 0 & 0 & 0 & 0 \\
\hline $\begin{array}{l}\text {... mögen manches und lehnen } \\
\text { manches ab. }\end{array}$ & 0 & 0 & 0 & O & 0 \\
\hline ... haben einen freien Willen. & 0 & 0 & 0 & 0 & 0 \\
\hline ... können sich freuen und leiden. & 0 & 0 & 0 & 0 & 0 \\
\hline $\begin{array}{l}\text {... haben eine eigene } \\
\text { Persönlichkeit. }\end{array}$ & 0 & 0 & 0 & 0 & 0 \\
\hline $\begin{array}{l}\text {... können niedrige und hohe } \\
\text { Lebensqualität unterscheiden. }\end{array}$ & 0 & 0 & 0 & 0 & 0 \\
\hline
\end{tabular}

Welche dieser Aussagen treffen Ihrer Meinung nach auf Nutztiere zu? Nutztiere... 


\begin{tabular}{|c|c|c|c|c|c|}
\hline & $\begin{array}{c}\text { Nein, } \\
\text { lehne ab } \\
1 \\
\end{array}$ & 2 & 3 & 4 & $\begin{array}{c}\text { Ja, } \\
\text { stimme zu } \\
5\end{array}$ \\
\hline ... verfolgen eigene Interessen. & 0 & 0 & 0 & 0 & 0 \\
\hline ... verhalten sich vernünftig. & 0 & 0 & 0 & O & 0 \\
\hline $\begin{array}{l}\text {... mögen manches und lehnen } \\
\text { manches ab. }\end{array}$ & O & $\mathrm{O}$ & $\mathrm{O}$ & 0 & O \\
\hline ... haben einen freien Willen. & ○ & O & O & O & O \\
\hline ... können sich freuen und leiden. & $\bigcirc$ & O & O & O & O \\
\hline 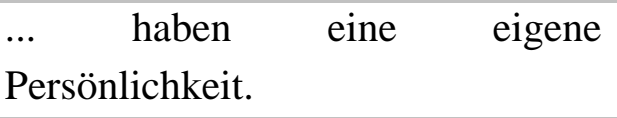 & O & O & O & O & O \\
\hline $\begin{array}{l}\text {... können niedrige und hohe } \\
\text { Lebensqualität unterscheiden. }\end{array}$ & O & O & O & O & O \\
\hline
\end{tabular}


In dem folgen Frageblock soll Ihre Einstellung zur aktuellen Tierhaltung erfasst werden.

\begin{tabular}{|c|c|c|c|c|c|}
\hline & $\begin{array}{l}\text { Nein, } \\
\text { lehne ab } \\
1\end{array}$ & 2 & 3 & 4 & $\begin{array}{c}\text { Ja, stimme } \\
\text { zu } \\
5\end{array}$ \\
\hline $\begin{array}{l}\text { Die Tierhaltung in der } \\
\text { konventionellen Landwirtschaft in } \\
\text { Deutschland ist artgerecht. }\end{array}$ & 0 & 0 & 0 & 0 & 0 \\
\hline $\begin{array}{l}\text { Das Wohlbefinden der } \\
\text { hat Eutztiere } \\
\text { Lebensqualität. }\end{array}$ & 0 & 0 & 0 & 0 & 0 \\
\hline $\begin{array}{l}\text { Das Haltungssystem von } \\
\text { landwirtschaftlichen Nutztieren ist } \\
\text { verbesserungswürdig. }\end{array}$ & 0 & 0 & 0 & 0 & 0 \\
\hline $\begin{array}{l}\text { Vorlieben von Nutztieren sollten } \\
\text { bei Tierschutzentscheidungen } \\
\text { berücksichtigt werden. }\end{array}$ & 0 & 0 & 0 & 0 & 0 \\
\hline $\begin{array}{l}\text { Für Nutztiere spielt das } \\
\text { Wohlbefinden eine ähnliche Rolle } \\
\text { wie für uns Menschen. }\end{array}$ & 0 & 0 & 0 & 0 & 0 \\
\hline $\begin{array}{l}\text { Ich bin für artgerechte Haltung von } \\
\text { Nutztieren. }\end{array}$ & 0 & 0 & 0 & 0 & 0 \\
\hline $\begin{array}{l}\text { Nutztiere empfinden Leid und } \\
\text { Schmerz in ähnlicher Form wie } \\
\text { Menschen. }\end{array}$ & 0 & 0 & 0 & 0 & 0 \\
\hline $\begin{array}{l}\text { Ich halte die EU Vorschriften zu } \\
\text { Haltungsbedingungen von } \\
\text { landwirtschaftlichen Nutztieren für } \\
\text { ausreichend. }\end{array}$ & 0 & 0 & 0 & 0 & 0 \\
\hline $\begin{array}{l}\text { Das Wohlbefinden von Nutztieren } \\
\text { ist mir wichtig. }\end{array}$ & 0 & 0 & 0 & 0 & 0 \\
\hline
\end{tabular}




\section{Präferenztest bei Kühen}

In der Wissenschaft werden Präferenztests durchgeführt, um herauszufinden wie Tiere auf verschiedene Haltungsbedingungen reagieren.

In einem Präferenztest mit Kühen wurden drei verschiedene Bodenbeläge getestet. Der erste Belag bestand aus tief eingestreuten Sägespänen, der zweite aus tief eingestreutem Sand. Die dritte Möglichkeit war eine wasserdurchlässige Matte aus natürlichem Material, welche mit 2-3 cm Sägespäne bestreut war.

Die Kühe haben zunächst freien Zugang zu allen drei Bodenbelägen. Dabei wird beobachtet, wie häufig sich die Tiere auf den verschiedenen Belägen hinlegen. Danach werden die Kühe einzeln für jeweils 2 Tage auf jedem der drei Beläge beobachtet, bevor sie wieder freien Zugang zu allen Bodenbelägen erhalten.

10 von 12 Kühen hielten sich am häufigsten auf den tief eingestreuten Sägespänen auf. Bei der Haltung auf Sandboden war auffällig, dass sich die Kühe deutlich weniger und kürzer hinlegten. Am wenigsten ruhten sich die Kühe auf der wasserdurchlässigen Matte aus.

\begin{tabular}{|c|c|c|c|c|c|}
\hline & $\begin{array}{c}\text { Nein, } \\
\text { lehne ab } \\
1\end{array}$ & 2 & 3 & 4 & $\begin{array}{c}\text { Ja, stimme } \\
\mathrm{zu} \\
5\end{array}$ \\
\hline $\begin{array}{l}\text { Die Auswahl, die das Nutztier in } \\
\text { diesem Test trifft, ist zufällig. }\end{array}$ & 0 & 0 & 0 & 0 & 0 \\
\hline $\begin{array}{l}\text { Verschiedene } \\
\text { Auswahlmöglichkeiten sind einem } \\
\text { Nutztier unterschiedlich wichtig. }\end{array}$ & 0 & 0 & 0 & 0 & 0 \\
\hline $\begin{array}{l}\text { Auswahlmöglichkeiten, die dem } \\
\text { Nutztier wichtig sind, werden } \\
\text { häufiger aufgesucht. }\end{array}$ & 0 & 0 & 0 & 0 & 0 \\
\hline $\begin{array}{l}\text { Haltungssysteme von Nutztieren } \\
\text { können durch diesen Test } \\
\text { verbessert werden. }\end{array}$ & 0 & 0 & 0 & 0 & 0 \\
\hline $\begin{array}{l}\text { Wenn sich das Nutztier häufig für } \\
\text { eine Auswahlmöglichkeit } \\
\text { entscheidet, sollte diese den } \\
\text { Nutztieren zur Verfügung gestellt } \\
\text { werden. }\end{array}$ & 0 & 0 & 0 & 0 & 0 \\
\hline $\begin{array}{l}\text { Das Wohlbefinden von Tieren in } \\
\text { der Nutztierhaltung kann durch } \\
\text { diesen Test verbessert werden. }\end{array}$ & 0 & 0 & 0 & 0 & 0 \\
\hline
\end{tabular}




\section{Präferenztest bei Nerzen}

In der Wissenschaft werden Präferenztests durchgeführt, um herauszufinden wie

Tiere auf verschiedene Haltungsbedingungen reagieren.

Einem Nerz (gezüchtet wegen des Pelzes) werden gleichzeitig sieben Räume mit jeweils einer anderen Beschäftigungsmöglichkeit zur Verfügung gestellt. In den Räumen findet der Nerz beispielsweise ein Schwimmbecken, ein Nest, eine Plattform, ein Spielzeug oder einen leeren Raum für mehr Platzangebot.

Während der Nerz freien Zugang zu allen Räumen hat, wird gezählt, wie häufig er die einzelnen Möglichkeiten aufsucht, wie viel Zeit er jeweils mit diesen verbringt und welches Verhalten er zeigt.

Ein solcher Test wurde nacheinander mit mehreren Tieren durchgeführt. Die Auswertung zeigte, dass die Nerze das Schwimmbecken am häufigsten und längsten aufsuchten. Der leere Raum war für die Tiere am wenigsten interessant.

\begin{tabular}{|c|c|c|c|c|c|}
\hline & $\begin{array}{c}\text { Nein, } \\
\text { lehne ab } \\
1 \\
\end{array}$ & 2 & 3 & 4 & $\begin{array}{l}\text { timme } \\
\mathrm{zu} \\
5\end{array}$ \\
\hline $\begin{array}{l}\text { Die Auswahl, die das Nutztier in } \\
\text { diesem Test trifft, ist zufällig. }\end{array}$ & 0 & 0 & 0 & 0 & 0 \\
\hline $\begin{array}{l}\text { Verschiedene } \\
\text { Auswahlmöglichkeiten sind einem } \\
\text { Nutztier unterschiedlich wichtig. }\end{array}$ & 0 & 0 & 0 & 0 & 0 \\
\hline $\begin{array}{l}\text { Auswahlmöglichkeiten, die dem } \\
\text { Nutztier wichtig sind, werden } \\
\text { häufiger aufgesucht. }\end{array}$ & 0 & 0 & 0 & 0 & 0 \\
\hline $\begin{array}{l}\text { Haltungssysteme } \\
\text { können durch } \\
\text { verbessert werden. }\end{array}$ & 0 & 0 & 0 & 0 & 0 \\
\hline $\begin{array}{l}\text { Wenn sich das Nutztier häufig für } \\
\text { eine Auswahlmöglichkeit } \\
\text { entscheidet, sollte diese den } \\
\text { Nutztieren zur Verfügung gestellt } \\
\text { werden. }\end{array}$ & 0 & 0 & 0 & 0 & 0 \\
\hline $\begin{array}{l}\text { Das Wohlbefinden von Tieren in } \\
\text { der Nutztierhaltung kann durch } \\
\text { diesen Test verbessert werden. }\end{array}$ & 0 & 0 & 0 & 0 & 0 \\
\hline
\end{tabular}




\section{Motivationstest bei Kühen}

In der Wissenschaft werden Motivationstests durchgeführt, um herauszufinden, wie Tiere auf verschiedene Haltungsbedingungen reagieren.

In einem Motivationstest mit Kühen wurden drei verschiedene Bodenbeläge getestet. Der erste Belag bestand aus tief eingestreuten Sägespänen, der zweite aus tief eingestreutem Sand. Die dritte Möglichkeit war eine wasserdurchlässige Matte aus natürlichem Material, welche mit 2-3 cm Sägespäne bestreut war.

Die Kühe haben zunächst freien Zugang zu allen drei Bodenbelägen. Dabei wird beobachtet, wie häufig sich die Tiere auf den verschiedenen Belägen hinlegen. Danach wird vor die einzelnen Buchten eine Schranke angebracht, welche die Kühe durch Drücken beiseiteschieben können. Nach jedem Durchqueren der Tür wird die Schranke mit zusätzlichen Gewichten beschwert, so dass es für die Kuh immer schwieriger wird in die Bucht zu gelangen. Wenn die Kuh die Energie nicht mehr aufwendet, um die Schranke zur Seite zu schieben ist die Motivationsgrenze erreicht.

Für die Bucht mit den tief eingestreuten Sägespänen brachten die Kühe am meisten Energie auf, für die wasserdurchlässige Matte aus natürlichem Material am weniosten

\begin{tabular}{|c|c|c|c|c|c|}
\hline & $\begin{array}{c}\text { Nein, } \\
\text { lehne ab } \\
1 \\
\end{array}$ & 2 & 3 & 4 & $\begin{array}{l}\text { stimme } \\
\mathrm{zu} \\
5 \\
\end{array}$ \\
\hline $\begin{array}{l}\text { Die Auswahl, die das Nutztier in } \\
\text { diesem Test trifft, ist zufällig. }\end{array}$ & 0 & 0 & 0 & 0 & 0 \\
\hline $\begin{array}{l}\text { Verschiedene } \\
\text { Auswahlmöglichkeiten sind einem } \\
\text { Nutztier unterschiedlich wichtig. }\end{array}$ & 0 & 0 & 0 & 0 & 0 \\
\hline $\begin{array}{l}\text { Auswahlmöglichkeiten, die dem } \\
\text { Nutztier wichtig sind, werden } \\
\text { häufiger aufgesucht. }\end{array}$ & 0 & 0 & 0 & 0 & 0 \\
\hline $\begin{array}{l}\text { Haltungssysteme } \\
\text { können durch diesen } \\
\text { verbessert werden. }\end{array}$ & 0 & 0 & 0 & 0 & 0 \\
\hline $\begin{array}{l}\text { Wenn sich das Nutztier häufig für } \\
\text { eine Auswahlmöglichkeit } \\
\text { entscheidet, sollte diese den } \\
\text { Nutztieren zur Verfügung gestellt } \\
\text { werden. }\end{array}$ & 0 & 0 & 0 & 0 & 0 \\
\hline $\begin{array}{l}\text { Das Wohlbefinden von Tieren in } \\
\text { der Nutztierhaltung kann durch }\end{array}$ & 0 & 0 & 0 & 0 & 0 \\
\hline
\end{tabular}


diesen Test verbessert werden.

\section{Motivationstest bei Nerzen}

In der Wissenschaft werden Motivationstests durchgeführt, um herauszufinden, wie Tiere auf verschiedene Haltungsbedingungen reagieren.

Einem Nerz (gezüchtet wegen des Pelzes) wurden gleichzeitig sieben Räume mit verschiedenen Beschäftigungsmöglichkeiten zur Verfügung gestellt. In den Räumen fand der Nerz beispielsweise ein Schwimmbecken, ein Nest, eine Plattform oder einen leeren Raum für mehr Platzangebot. Vor den Räumen wurden Türen angebracht, die der Nerz durch Drücken überwinden musste, um an sein Ziel zu gelangen.

Jedes Mal, wenn der Nerz erfolgreich die Tür geöffnet hatte wurde diese mit Gewichten beschwert, so dass der Nerz beim nächsten Mal noch mehr Energie aufbringen musste, um an das Objekt hinter der Tür zu gelangen. Bringt der Nerz die Energie nicht mehr auf, um an das Objekt hinter der Tür zu gelangen, ist seine Motivationsgrenze erreicht.

Im Durchschnitt brachten die Nerze für das Schwimmbecken am meisten Energie auf und für einen leeren Raum am wenigsten.

\begin{tabular}{|c|c|c|c|c|c|}
\hline & $\begin{array}{l}\text { Nein, } \\
\text { lehne ab } \\
1 \\
\end{array}$ & 2 & 3 & 4 & $\begin{array}{l}\text { sume } \\
\mathrm{zu} \\
5\end{array}$ \\
\hline $\begin{array}{l}\text { Die Auswahl, die das Nutztier in } \\
\text { diesem Test trifft, ist zufällig. }\end{array}$ & 0 & 0 & 0 & 0 & 0 \\
\hline $\begin{array}{l}\text { Verschiedene } \\
\text { Auswahlmöglichkeiten sind einem } \\
\text { Nutztier unterschiedlich wichtig. }\end{array}$ & 0 & 0 & 0 & 0 & 0 \\
\hline $\begin{array}{l}\text { Auswahlmöglichkeiten, die dem } \\
\text { Nutztier wichtig sind, werden } \\
\text { häufiger aufgesucht. }\end{array}$ & 0 & 0 & 0 & 0 & 0 \\
\hline $\begin{array}{l}\text { Haltungssysteme } \\
\text { können durch diesen Tutztieren } \\
\text { verbessert werden. }\end{array}$ & 0 & $\bigcirc$ & 0 & 0 & 0 \\
\hline $\begin{array}{l}\text { Wenn sich das Nutztier häufig für } \\
\text { eine Auswahlmöglichkeit } \\
\text { entscheidet, sollte diese den } \\
\text { Nutztieren zur Verfügung gestellt } \\
\text { werden. }\end{array}$ & 0 & 0 & 0 & $\bigcirc$ & 0 \\
\hline Das Wohlbefinden von Tieren in & 0 & 0 & 0 & 0 & 0 \\
\hline
\end{tabular}


der Nutztierhaltung kann durch

diesen Test verbessert werden.

In den folgenden Fragen geht es um Ihr Einkaufsverhalten

Wie viel zahlen Sie üblicherweise für 11 Milch?

Euro

Wie viel würde Sie für diesen Liter Milch mehr zahlen, wenn die Milch von einem Betrieb kommt, dessen Tierhaltung auf den Ergebnissen von Präferenzund Motivationstests beruht?

$0 \mathrm{ct}+10 \mathrm{ct}+20 \mathrm{ct}+30 \mathrm{ct}+40 \mathrm{ct}+50 \mathrm{ct}+60 \mathrm{ct}+70 \mathrm{ct}+80 \mathrm{ct}+90 \mathrm{ct}+1 €$

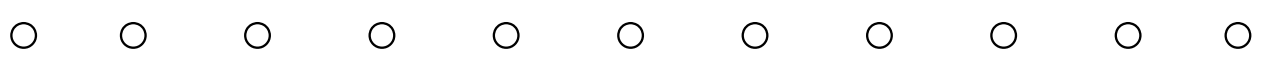

Ich kaufe keine Milch

Wie viel zahlen Sie üblicherweise für 6 Eier Milch?

Euro

Wie viel würde Sie für diese 6 Eier mehr zahlen, wenn die Milch von einem Betrieb kommt, dessen Tierhaltung auf den Ergebnissen von Präferenz- und Motivationstests beruht?

$0 \mathrm{ct}+10 \mathrm{ct}+20 \mathrm{ct}+30 \mathrm{ct}+40 \mathrm{ct}+50 \mathrm{ct}+60 \mathrm{ct}+70 \mathrm{ct}+80 \mathrm{ct}+90 \mathrm{ct}+1 €$

$\begin{array}{llllllllll}0 & 0 & 0 & 0 & 0 & 0 & 0 & 0 & 0 & 0\end{array}$

Ich kaufe keine Eier 
Wie viel zahlen Sie üblicherweise für 250 g Hackfleisch?

$$
\text { Euro }
$$

Wie viel würde Sie für diese 250 g Hackfleisch mehr zahlen, wenn die Milch von einem Betrieb kommt, dessen Tierhaltung auf den Ergebnissen von Präferenz- und Motivationstests beruht?

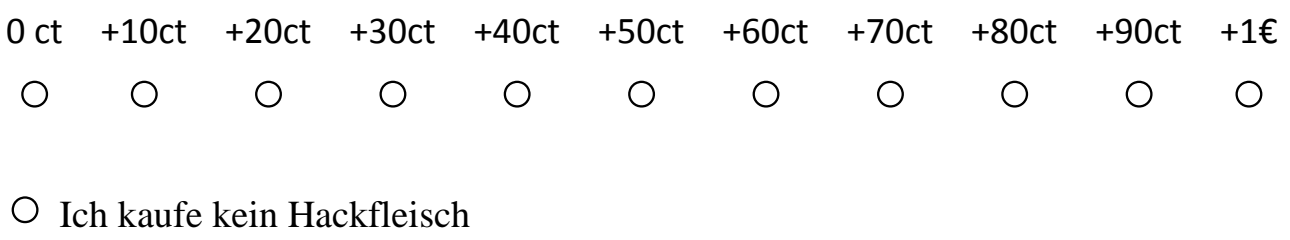


Im Folgenden beschreibe ich Ihnen einige Personen. Bitte kreuzen Sie in jeder Zeile an, wie ähnlich oder unähnlich Ihnen diese Person ist.

\begin{tabular}{|c|c|c|c|c|c|c|}
\hline & $\begin{array}{c}\text { Ist mir } \\
\text { überhaupt } \\
\text { nicht ähnlich }\end{array}$ & $\begin{array}{l}\text { Ist mir } \\
\text { nicht } \\
\text { ähnlich }\end{array}$ & $\begin{array}{l}\text { Ist mir nur ein } \\
\text { kleines bisschen } \\
\text { ähnlich }\end{array}$ & $\begin{array}{l}\text { Ist mir } \\
\text { etwas } \\
\text { ähnlich }\end{array}$ & $\begin{array}{l}\text { Ist mir } \\
\text { ähnlich }\end{array}$ & $\begin{array}{l}\text { Ist mir } \\
\text { sehr } \\
\text { ähnlich }\end{array}$ \\
\hline $\begin{array}{l}\text { Der Person ist wichtig, neue Ideen zu entwickeln und } \\
\text { kreativ zu sein. Sie macht Sachen gerne auf ihre } \\
\text { eigene originelle Art }\end{array}$ & 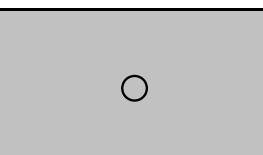 & 0 & 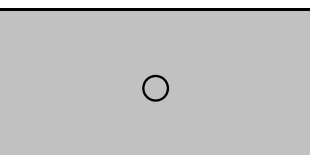 & 0 & 0 & 0 \\
\hline $\begin{array}{l}\text { Dieser Person ist wichtig, reich zu sein. Sie möchte } \\
\text { viel Geld haben und teure Sachen besitzen. }\end{array}$ & 0 & 0 & 0 & 0 & 0 & 0 \\
\hline $\begin{array}{l}\text { Diese Person hält für wichtig, dass alle Menschen auf } \\
\text { der Welt gleich behandelt werden sollten. Sie glaubt, } \\
\text { dass jeder Mensch im Leben gleiche Chancen haben } \\
\text { sollte. }\end{array}$ & 0 & 0 & 0 & 0 & 0 & 0 \\
\hline $\begin{array}{l}\text { Dieser Person ist wichtig, ihre Fähigkeiten zu zeigen. } \\
\text { Sie möchte, dass die Leute bewundern was sie tut. }\end{array}$ & 0 & 0 & 0 & 0 & 0 & 0 \\
\hline $\begin{array}{l}\text { Dieser Person ist wichtig, in einem sicheren Umfeld } \\
\text { zu leben. Sie vermeidet alles, was ihre Sicherheit } \\
\text { gefährden könnte. }\end{array}$ & 0 & 0 & 0 & 0 & 0 & 0 \\
\hline $\begin{array}{l}\text { Diese Person glaubt, dass die Menschen tun sollten, } \\
\text { was man Ihnen sagt. Sie denkt, dass Menschen sich } \\
\text { an Regeln halten sollten, selbst dann wenn es } \\
\text { niemand sieht. }\end{array}$ & 0 & 0 & 0 & 0 & 0 & 0 \\
\hline
\end{tabular}




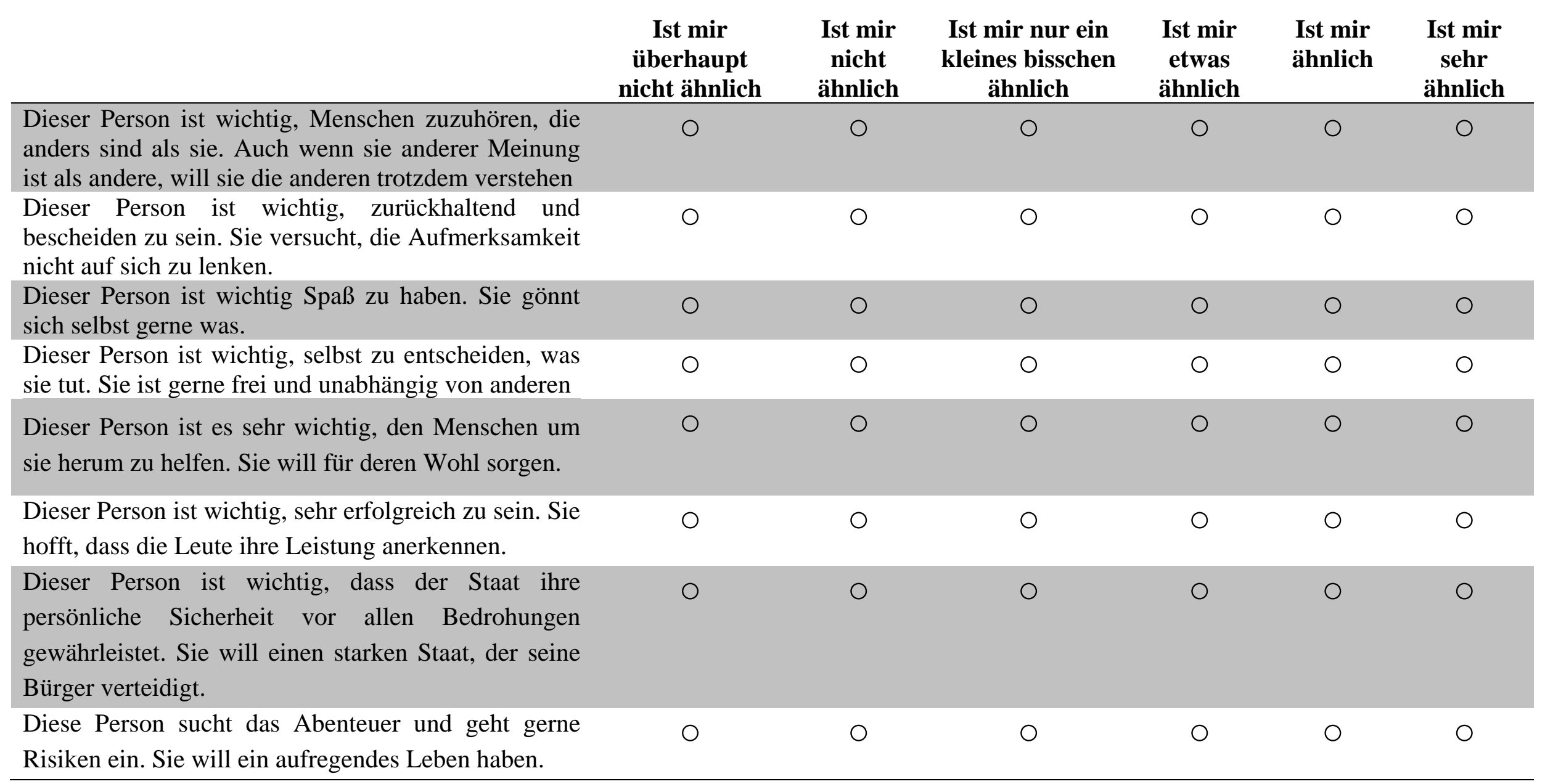




\begin{tabular}{|c|c|c|c|c|c|c|}
\hline & $\begin{array}{c}\text { Ist mir } \\
\text { überhaupt } \\
\text { nicht ähnlich }\end{array}$ & $\begin{array}{l}\text { Ist mir } \\
\text { nicht } \\
\text { ähnlich }\end{array}$ & $\begin{array}{c}\text { Ist mir nur ein } \\
\text { kleines bisschen } \\
\text { ähnlich }\end{array}$ & $\begin{array}{c}\text { Ist mir } \\
\text { etwas } \\
\text { ähnlich }\end{array}$ & $\begin{array}{l}\text { Ist mir } \\
\text { ähnlich }\end{array}$ & $\begin{array}{c}\text { Ist mir } \\
\text { sehr } \\
\text { ähnlich }\end{array}$ \\
\hline $\begin{array}{l}\text { Dieser Person ist wichtig, sich jederzeit korrekt zu } \\
\text { verhalten. Sie vermeidet es, Dinge zu tun, die } \\
\text { andere Leute für falsch halten könnten }\end{array}$ & 0 & 0 & 0 & 0 & 0 & 0 \\
\hline $\begin{array}{l}\text { Dieser Person ist wichtig, dass andere sie } \\
\text { respektieren. Sie will, dass die Leute tun, was sie } \\
\text { sagt }\end{array}$ & 0 & 0 & 0 & 0 & 0 & 0 \\
\hline $\begin{array}{l}\text { Diese Person ist fest davon überzeugt, dass die } \\
\text { Menschen sich um die Natur kümmern sollten. } \\
\text { Umweltschutz ist ihr wichtig. }\end{array}$ & 0 & 0 & 0 & 0 & 0 & 0 \\
\hline $\begin{array}{l}\text { Dieser Person ist Tradition wichtig. Sie versucht, } \\
\text { sich an die Sitten und Gebräuche zu halten, die ihr } \\
\text { von ihrer Religion oder ihrer Familie überliefert } \\
\text { wurden. }\end{array}$ & 0 & 0 & 0 & 0 & 0 & 0 \\
\hline $\begin{array}{l}\text { Diese Person lässt keine Gelegenheit aus, Spaß zu } \\
\text { haben. Es ist ihr wichtig Dinge zu tun, die ihr } \\
\text { Vergnügen bereiten. }\end{array}$ & 0 & 0 & 0 & 0 & 0 & 0 \\
\hline
\end{tabular}




\section{Angaben zur Person}

männlich

$\bigcirc$ weiblich

In welchem Jahr sind Sie geboren?

Welches Fach haben Sie zuletzt studiert oder studieren Sie?

Bitte geben Sie ihr Hauptfach an oder wählen Sie ein Fach als Ihr Hauptfach aus.

$\begin{array}{ll}\bigcirc & \text { Agrarwissenschaften } \\ \bigcirc & \text { ökologische Agrarwissenschaften } \\ \bigcirc & \text { Biologie } \\ \bigcirc & \text { Psychologie } \\ \bigcirc & \text { Betriebswirtschaftslehre } \\ \bigcirc & \text { Volkswirtschaftslehre } \\ \bigcirc & \text { Jura } \\ \bigcirc & \text { Philosophie } \\ \bigcirc & \text { Landschaftsökologie }\end{array}$

Wenn ihr Studienfach nicht in der Liste aufgeführt ist tragen Sie dieses bitte hier ein:

In welcher Phase des Studiums befinden Sie sich oder haben Sie sich zuletzt befunden?

Bachelor, Vordiplom, 1.Staatsexamen

O Master, Diplom, 2.Staatsexamen

O Abgeschlossener Master, Promotion

Was trifft auf Sie zu? 
Nein,

lehne ab

Ich sehe mich selbst als typisches

Mitglied dieses Studienganges.

Ich bin froh, dieses Fach gewählt zu haben.

Ich fühle eine starke Bande zu dem

Berufsbild dieses Fachs.

Ich identifiziere mich mit anderen, die dieses Fach studiert haben. 1

Ja, stimme zu 5

00000

$\begin{array}{lllll}0 & 0 & 0 & 0 & 0\end{array}$

$\bigcirc$

○ 0

$\begin{array}{llll}0 & 0 & 0\end{array}$

0

Wie häufig essen Sie durchschnittlich in der Woche Fleisch und Wurst?

$\begin{array}{cc}\bigcirc & \text { gar nicht } \\ 0 & 1-2 \mathrm{mal} \\ 0 & 3-4 \mathrm{mal} \\ \bigcirc & 5-6 \mathrm{mal} \\ \bigcirc & \text { täglich }\end{array}$

Essen Sie im Vergleich zu anderen Studierenden wenig oder viel Fleisch?

wenig

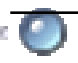

Haben Sie zum Thema dieses Fragebogens noch einen Kommentar?

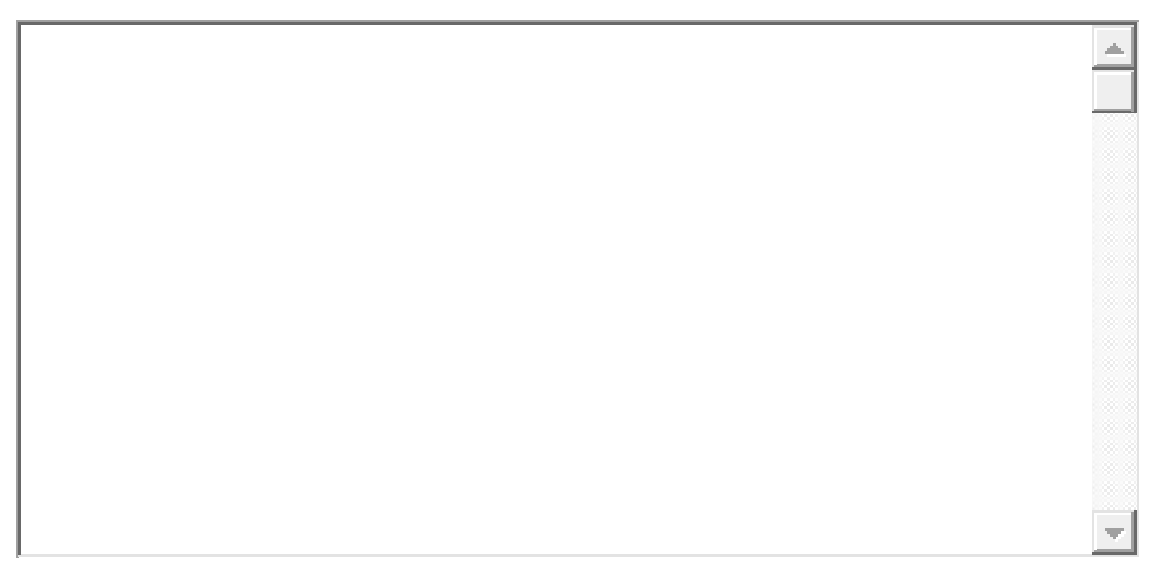

Vielen Dank, dass Sie sich die Zeit genommen haben um an der Umfrage teilzunehmen! 
Ihre Antworten wurden gespeichert, Sie können das Fenster nun schließen!

Sollten Sie Fragen oder weitere Kommentare zum Thema des Fragebogens haben, nutzen Sie bitte die folgende Email Adresse:

kgrevin@uni-goettingen.de 


\section{Erklärungen}

1. Hiermit erkläre ich, dass diese Arbeit weder in gleicher noch in ähnlicher Form bereits anderen Prüfungsbehörden vorgelegen hat.

Weiter erkläre ich, dass ich mich an keiner andern Hochschule um einen Doktorgrad beworben habe.

Göttingen, den 11.12.2014

(Unterschrift)

2. Hiermit erkläre ich eidesstattlich, dass diese Dissertation selbstständig und ohne unerlaubte Hilfe angefertigt wurde.

Göttingen, den 11.12.2014

(Unterschrift) 